\title{
Unconventional carrier transport and switching with Graphene pn junction
}

\footnotetext{
A Dissertation

Presented to

the Faculty of the School of Engineering and Applied Science

University of Virginia

In Partial Fulfillment

of the requirements for the Degree

Doctor of Philosophy (Electrical and Computer Engineering)

by

Redwan Noor Sajjad

December 2014
} 
APPROVAL SHEET

The dissertation

is submitted in partial fulfillment of the requirements

for the degree of

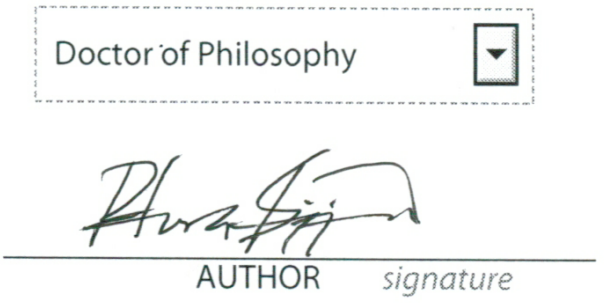

The dissertation has been read and approved by the examining committee:

Please insert committee member names below:

Avik Ghosh

Advisor

Robert M. Weikle

Eugene Kolomeisky

Lloyd R. Harriott

Joe C. Campbell

Accepted for the School of Engineering and Applied Science:

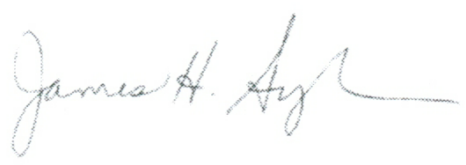

Dean, School of Engineering and Applied Science

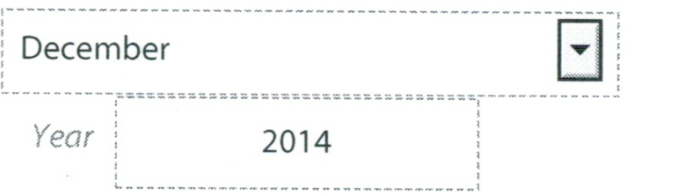




\section{Acknowledgments}

I would like to thank my advisor Professor Avik Ghosh for his continuous guidance and patience to guide my research effectively and wholeheartedly. I received valuable advice not just on the particular research area I worked on, but on how to succeed as a researcher, which will help me for my lifetime.

I would very much like to acknowledge countless hours of intriguing discussions with my current and former colleagues in Virginia Nano-Computing (VINO) research group: Carlos Polanco, Frank Tseng, Yunkun Xie and K. M. Masum Habib. Without the lively and enthusiastic discussions (and sometimes arguments), I wouldn't get the valuable insight that I achieved over the years. I also appreciate my collaborators in other Universities, Prof. J. U. Lee (SUNY, Albany), Prof. Azad Naeemi (Georgia Tech), Chenyun Pan (Georgia Tech) for very effective collaboration and discussions.

A substantial part of the research was the computational study on graphene based nanoelectronic devices. To perform the simulations, I used the computational cluster maintained by University of Virginia Advanced Computing Services and Engagement (UVACSE). I appreciate the service provided by them and the answers to numerous questions I asked to be able to effectively use the cluster.

Finally I would like to appreciate all the love, support and patience of my family members: my parents, sisters and my wife throughout my academic life. I attribute all my achievements to them.

This work was supported by the financial grant from Institute for Nanoelectronics Discovery 
and Exploration (INDEX) center, part of Nanoelectronics Research Initiative (NRI) of Semiconductor Research Corporation (SRC). 
To my family 


\section{Abstract}

Graphene is considered to be a wonder material for its unique physical properties. In graphene, record-breaking numbers have been shown for the thermal and electrical conductivities, mechanical strength, electronic mobility, chemical sensing, filtering and optoelectronic properties. Therefore, it has potential for various electronic, spintronic and photonic applications. In this dissertation, we investigate graphene's potential as a channel material for digital logic applications using electro-statically built graphene pn junction (GPNJ). Despite graphene's high electrical conductivity and other useful properties, the lack of bandgap makes it difficult to accomplish logic implementation, which requires a large amount of current modulation with gate voltage. In graphene $p n$ junction, the linear, photon like energy dispersion combined with zero bandgap leads to an electron transport much like optical refraction and carrier trajectories are governed by an equivalent Snell's law. Determined by the wave-function dynamics, it also has a unique angle (transverse mode) dependent transmission through a pn junction. This research aims to manipulate such angle dependent transport with gate geometry for switching. We show that such scheme is capable of switching without having to open a structural bandgap, but with what we call a 'Transmission Gap'. We show multiple device concepts that manipulate the angle dependent transmission with device geometry and produce the gap by suppressing transmission of all propagating modes. The tunability of the gap leads to a new way of beating thermal switching limit. Combined with graphene's high current carrying capability, these properties make the switch energy efficient. The device designs are complemented with our benchmarking of recent experiments on angle 
dependent transport in GPNJ. We also show an intriguing implication of the $p n$ junction based conductance control in another novel material: topological insulator (TI) which has similar bandstructure on its surface as graphene. A TI based $p n$ junction is shown to produce high spin current with low charge current, following a very similar tunneling physics in GPNJ. Such gate controlled spin current can have implication in spin based systems, where a spin polarized current is needed to rotate a ferromagnet with as low charge current as possible to decrease dissipation. Throughout the dissertation, we show simulation results from a sophisticated quantum mechanical numerical modeling platform based upon Non-Equilibrium Green's Function (NEGF) formalism, developed to augment the analytical formalism. The numerical platform is optimized so that it can perform calculations that the analytical model cannot possibly do: model all kinds of transport (e.g. electron, spin) for small to large scale devices (up to experimental device size) for both ballistic and diffusive transport regime including non-idealities such as charge impurity scattering and edge effects. 


\section{Contents}

Contents g

List of Figures . . . . . . . . . . . . . . . . . . . . i i

1 Introduction 1

1.1 Graphene: fundamentals, potentials and challenges . . . . . . . . . . 1

1.1.1 Electronic properties of graphene . . . . . . . . . . . . . 2

1.1.2 Silicon CMOS and graphene based transistors . . . . . . . . . . . 5

1.1.3 How close are we to realizing ballistic transport? . . . . . . . . . . . 7

1.2 Controlling electron flow in graphene pn junction . . . . . . . . . . . . . 9

1.3 Overview of the dissertation . . . . . . . . . . . . . . . 11

2 Transport physics of graphene pn junction $\quad 12$

2.1 Angle dependent tunneling in GPNJ . . . . . . . . . . . . . . . . . . . . . 12

2.1.1 Carrier trajectories: Snell's law for graphene . . . . . . . . . . . . . 12

2.1.2 Transmission probability: Fresnel's equation for graphene . . . . . . . 15

2.2 Controlling GPNJ conductance with gate parameters . . . . . . . . . . . 20

2.2.1 Conductance of smooth pn junction: low pass filter . . . . . . . . . . 20

2.2.2 Conductance of a tilted $p n$ junction: high pass filter . . . . . . . . . . 24

3 Transmission gap: a new way of switching 33

3.1 Opening transmission gap with gate geometry . . . . . . . . . . . . . 34

3.2 Biasing scheme and impact on subthreshold slope . . . . . . . . . . . . . . 39

3.3 NEGF simulation with edge effects: large scale graphene . . . . . . . . . . 41

3.4 NEGF simulation without edge effects: smaller structures with open boundary condition . . . . . . . . . . . . . . . . . . . . . 42

3.5 Removing Klein tunnel mode with external barrier . . . . . . . . . . . . 43

4 What we can do with pseudospin(spin) filtering by pn junction 49

4.1 GPNJ as a Boolean logic device . . . . . . . . . . . . . . . . . 49

4.2 Spin filtering in topological insulators . . . . . . . . . . . . . . . 51

5 Non-Equilibrium Green's Function (NEGF) formalism: a rigorous transport model $\quad 57$

5.1 NEGF: an efficient Schrödinger solver . . . . . . . . . . . . . . . 57

5.1 .1 Modified Schrödinger equation . . . . . . . . . . . . 58 
5.1.2 Broadened density of states and electron escape rate . . . . . . . . 59

5.1.3 Current and transmission: Landauer formula . . . . . . . . . . . . . 61

5.2 Ballistic electron transport in graphene . . . . . . . . . . . . . . . . . . 62

5.2.1 Electronic conductance . . . . . . . . . . . . . . . . . . 62

5.2.2 Conductance of a double gated structure . . . . . . . . . . . . 64

5.2.3 Modeling metal-graphene contact with NEGF . . . . . . . . . . . 65

5.2.4 Ballistic graphene FET output characteristics . . . . . . . . . . . . 68

5.3 Diffusive transport in graphene . . . . . . . . . . . . . . . . . 70

5.4 Magnetotransport . . . . . . . . . . . . . . . . . . . . 71

5.5 Spin transport . . . . . . . . . . . . . . . . . . 74

$\begin{array}{lll}6 & \text { Conclusion } & 75\end{array}$

$\begin{array}{ll}\text { A GPNJ transmission equations } & \mathbf{7 8}\end{array}$

A.1 Abrupt step potential (abrupt GPNJ) . . . . . . . . . . . . 78

A.2 Split gated structure: smooth variation of potential and collimation . . . . 85

B Transmission gap in multiple $p n$ junction device $\quad 89$

C Spin filtering in TI $p n$ junction $\quad 93$

C.1 Charge current density in TI . . . . . . . . . . . . . . . . . . . . 93

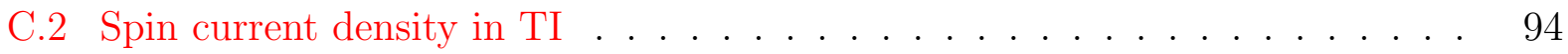

C.2.1 Spin current carried by $\mid \uparrow_{x}>\ldots \ldots . \ldots . \ldots . \ldots 9$

C.2.2 Spin current carried by $\mid \uparrow_{y}>\ldots \ldots . \ldots . \ldots . \ldots . . \ldots 96$

C.2.3 Spin current carried by $\mid \uparrow_{z}>\ldots \ldots . \ldots . \ldots . . \ldots 96$

C.3 Spin to charge current ratio . . . . . . . . . . . . . . . . . . . . 97

$\begin{array}{ll}\text { Bibliography } & 99\end{array}$ 


\section{List of Figures}

1.1 Graphene's honeycomb lattice structure. Amplitudes of Bloch functions on the A, B sites mimic two spin systems. They may be called pseudospins, which dictate the electronic properties such as suppressed backscattering. . . . . . 2

1.2 Graphene bandstructure . . . . . . . . . . . . . . . . . 3

1.3 Dispersion of graphene along different directions. There is no bandgap and $E-K$ is linear around the $K$ point. . . . . . . . . . . . . . . 4

1.4 Current voltage characteristics of CMOS and other technologies. Unless the transition rate $(60 \mathrm{mV} / \mathrm{Dec})$ of current is changed (desired $I_{D^{-}} V_{G}$ in purple), power dissipation $\left(0.5 C V_{D}^{2} f\right)$ and operating frequency $(f)$ cannot be improved.

1.5 Mobility degradation as bandgap is opened in graphene (figure from Frank Tseng) . . . . . . . . . . . . . . . . . . . .

1.6 Electron 'optics' at a graphene pn junction. Electron trajectories (below) mimic optical refraction and depend on electrostatic doping levels (above). .

2.1 Schematic of a graphene based $p n$ junction device with a point contact (much smaller than the width of the device) as an injector and a wide drain to collect the electrons. Electrostatic doping shifts graphene bands up/down depending on voltage sign. Bands cross at the Fermi energy, shown in red, enabling band to band tunneling. . . . . . . . . . . . . . . . .

2.2 Carrier trajectories in GPNJ, a) symmetric doping and focusing of electron flow, b) asymmetric doping where the refracted side has lower doping (smaller Fermi surface), electrons reflect back above a critical angle. . . . . . . . . . 14

2.3 Definition of $\theta$ in $k$ space. . . . . . . . . . . . . . . . . . . . . . 14

2.4 Matching wavefunctions on both sides gives transmission probability . . . . 16

2.5 Angle dependent transmission for various bias conditions. . . . . . . . . . 17

2.6 a) Average transmission per mode $T_{a v}$ and b) total conductance of GPNJ as a function of Fermi energy $E_{F}$. There are two zeros in the conductance plot signifying the two Dirac points in the system. . . . . . . . . . . 
2.7 Physics of npn junction. (a) Band diagram. The black line shows the change of Dirac point as a result of different dopings at different portions of the device. Blue (red) region in the E-k indicates empty (filled) states. (b) Electron trajectories in such junctions, multiple focusing takes place, (c) Ballistic conductance of a 50nm wide graphene sheet as a function of Fermi energy $\left(E_{F}\right)$ for a fixed barrier height $\left(V_{0}=1 \mathrm{eV}\right)$ but different barrier width, $D=5,25$ and 100nm (violet, red and black). The solid lines are from numerical NEGF calculation, while the circles are analytical calculation from Eq. 2.6, summed over all available modes. (d) Experimental data from Ref. [1]. Conductance in the experiment is much lower due to scattering processes. . . . . . . . . .

2.8 Impact of gate split on the angular transmission, electrons are collimated in a sense that only low angle electrons are transmitted. . . . . . . . . . . .

2.9 a) Conductance for various splits. b) How the conductance at the symmetric doping limit changes with gate split $\left(\sim \sqrt{k_{F} d / 2}\right) \ldots \ldots . \ldots$

2.10 Numerical calculation (NEGF) of a single $p n$ junction conductance for both abrupt and smooth GPNJ. (a-b) Variation of total resistance with dopings in the two regions for abrupt (a) and smooth potential (c) Resistance as a function of Fermi energy $\left(E_{F}\right)$ for a fixed built in potential $V_{0}=\Delta E_{1}-\Delta E_{2}=$ $0.2 \mathrm{eV}$. The red (abrupt) and black (smooth) lines are resistance plots along the corresponding black and red lines in (a) and (b). Conductance (inset) which can be viewed as $G=G_{0} M T_{a v}$ where $T_{a v}$, pinches off at two points - one due to vanishing $M$ and at another one due to vanishing $T_{a v}$, average transmission per mode. (d) The resistance asymmetry between $p n$ and $n n$ regime from plots along specific doping (specific $\Delta E_{1}$ lines from (b). (e) Extraction of $T_{a v}$ numerically. The transmission of a symmetric GPNJ is $2 / 3$ (at $\Delta E_{1}=0.1$ $\mathrm{eV}$ ), which results in asymmetric conductance vs doping in GPNJ. The solid lines are from NEGF and the circles are from analytical calculation, Eq. 2.11. (f) Junction resistance (Eq. 2.14) enhancement due to a tilt in the junction. This time we vary the doping along the diagonal (orange line in (b)) as done in the experiment $[2] \ldots \ldots \ldots \ldots \ldots$

2.11 (a) Experimental setup in Ref. [2] for two devices, no tilt (upper), 450 tilt (lower). The gate oxides are buried (runs vertically in the picture), (b) experimental and (c) theoretical calculation of junction resistance as a function of gate voltage. Resistance is higher for the tilted device compared to non tilted device, (d) Comparison of theoretical (solid lines, Eq. 2.13) and experimental (dots) average transmission per mode $T_{a v} \ldots \ldots \ldots$

(a) Angular transmission for various tilt angles. (b) With tilt, the transmission lobe moves into a low mode density $(\sim \cos \theta)$ area giving $(\mathrm{c})$ a gradual decrease in transmission (Eq. 2.13) for a symmetric pn junction, (d) junction resistance at $0 \mathrm{~K}$ predicted from Eq. 2.14. We see resonances which become more pronounced as we go into smaller systems. . . . . . . . . . . . . . .

2.13 Impact of charged impurity scattering, (a) conductance asymmetry is diluted due to impurity potentials, the ballistic resistance is normalized for comparison. (b) Reduced asymmetry results in lower junction resistance for both tilted, non-tilted devices, thus retaining their difference. . . . . . . . . . 
2.14 (a) Increase in conductance in a tilted GPNJ due to Edge Scattering (ES) in contrast with Fig. 2.12c, (b) corresponding decrease in junction resistance due to tilt (c) Mechanism of edge enhanced conductance for a tilted junction from atomistic NEGF calculation: some reflected electrons come back at the junction after edge reflection. . . . . . . . . . . . . . . . .

3.1 (a) Chiral tunneling in graphene pn junction (GPNJ) manipulated with gate geometry, using two junctions (dual GPNJ) tilted in opposite directions, (b) making their angle dependent transmission lobes orthogonal (left) and yielding negligible overall transmission (right) for split gates (solid line). (c) The transmission gap creates a high ON-OFF current ratio as a function of $V_{G 2}$ at finite bias, $V_{D S}=0.4 \mathrm{~V}$ and room temperature. The ON current degrades slightly compared to homogeneous gapless graphene, but the OFF current is reduced by several orders of magnitude (d) Steeper change (beating $K T \ln 10 / q$ switching limit) of current with $V_{G 3}$. This is done by keeping the collimation effect of the first junction intact and making the transmission gap dependent upon Snell's law. . . . . . . . . . . . . . . . . . . . .

3.2 Mode-averaged transmission $T_{a v}$ vs Fermi energy $E_{F}$ for different doping profiles (Fermi energy $E_{F}$ and built in potential $V_{0}$ are indicated on the top band diagram). $T_{a v}$ for the dual tilt GPNJ shows a gap (green line), which is termed as transmission gap (yellow shading) in this chapter. . . . . . . . .

3.3 Benchmarking $T_{a v}$ with experiment [2] for a single tilted split junction for several gate voltages. Experiment shows good agreement with the theory confirming the angular shift of $T(\theta)$ and the scaling law with tilt. . . . . . .

3.4 Electron flow in the proposed GPNJ device. Schematic of collimator-barrier pair that sequentially filters all propagating modes is shown on left. Numerical current density plot from NEGF showing (a) blocking of carrier flow in the bipolar npn OFF state, (b) unipolar $n n^{-} n$ ON state, current flowing from source to drain. White (black) areas indicate high (low) local current density.

3.5 Simulation of multiple GPNJ FET for large width. ON-OFF ratio improves with width while the smaller structures show degradation of performance due to edge effects. . . . . . . . . . . . . . . . . . .

3.6 Simulation of multiple GPNJ FET for small width but with open boundary condition at the edges. ON-OFF ratio is back up to $10^{3}$. . . . . . . . 
3.7 (a) Schematic diagram of the proposed device, showing a point source that angularly spreads electrons while an extended drain collects those that refract around a tunnel barrier. The point source could be lithographically patterned, an STM or electrostatically produced using analogous convex lens. Charge densities in the two segments created by the split gates are $\rho_{1}$ and $\rho_{2}$. (b) Energy band diagram for $\left|\mathrm{V}_{G 1}\right|>\left|\mathrm{V}_{G 2}\right|$. (c) With very small drain bias, atomistic current density plot calculated with Non-Equilibrium Green's function (NEGF) formalism illustrates that current flows only within the critical angle, $\theta_{C}=$ $\sin ^{-1}\left(\left|V_{G 2} / V_{G 1}\right|\right)$. (d) Schematic Snell's Law predictions as well as (e) NEGF simulation illustrate how a barrier (void in this case) eliminates transmission within the critical angle when $\theta_{C}<\theta_{B}$, where $\theta_{B}$ is the occlusion half angle created by the barrier. (f) Transmission is non zero for $\theta_{C}>\theta_{B}$ where electrons can refract around the barrier. . . . . . . . . . . . . . . . . .

3.8 GPNJ Transmission vs. Fermi energy (E) for $V_{G 1}-V_{G 2}=+0.8 \mathrm{~V}$. Unless $\rho_{2}$ is 0 , the normal mode always transmits perfectly, making the average transmission nonzero. (b) Constant Fermi energy slices of the Dirac cones across the junction, corresponding to points on the $T(E)$ curve with arrows indicating critical angle. (c) Modification of transmission from part (a) due to the barrier, transmission within the yellow region is forbidden (d) T vs $E_{L}$ at specific total energies $E$, indicating an effective band-pass behavior for states outside transmission gap. . . . . . . . . . . . . . . . . .

3.9 (a) Transmission shifts as we vary $V_{G 1}-V_{G 2}$, but is accompanied with a change in transmission gap (b) $\mathrm{I}_{-} \mathrm{V}_{G 2}$ of the device, showing a sharp increase in current modulation with barrier, and a subthreshold swing that is larger than the thermal limit at the PN end and smaller at the $\mathrm{N}^{+} \mathrm{N}$ end. Here, $\theta_{B}=20^{\circ}$ and drain bias $=0.2 \mathrm{~V}$.

4.1 Gate-Level GPNJ layout: (a) Inverter (b) NAND gate. Here, D is the gap distance of the GPNJ junction, and $\mathrm{F}$ is the minimum feature size, which is $16 \mathrm{~nm}$ in this work. . . . . . . . . . . . . . . . . .

4.2 The delay and power dissipation components of GPNJ logic circuits for inverter, NAND and adder. . . . . . . . . . . . . . . . 51

4.3 spin (pseudospin) locking in graphene (a) and topological insulator (b). . .

4.4 Spin Hall effect, where the charge current is accompanied with a transverse spin current. . . . . . . . . . . . . . . . . . .

4.5 (a) Cross section and (b) top view of 3D TI pn junction with incident, reflected and transmitted electron waves. The long arrows indicate the direction of group velocity and wavevector at a given energy $-q V_{n}<E<q V_{p}$. The short arrows indicate the direction of spin. (c) Potential profile of the device. For simplicity, linear potential profile is assumed between $n$ and $p$ regions. . . .

4.6 Normalized charge and spin current as functions of gate voltage $V_{p}$ for a device with width $W=100 \mathrm{~nm}$, length $L=120 \mathrm{~nm}$ and split length $2 d=100 \mathrm{~nm}$, drain bias $V_{D}=0.1 \mathrm{~V}$ and gate voltage $V_{n}=0.15 \mathrm{~V}$. Thus, built in potential $V_{0}=0.3 \mathrm{eV}$ when $V_{p}=0.15 \mathrm{~V} \ldots \ldots \ldots \ldots$

4.7 Spin Hall angle $\theta_{H}$ as a function of $V_{p} \ldots \ldots \ldots \ldots$ 
5.1 Device Hamiltonian $[H]$, contact information (self-energy $[\Sigma]$ and electrochemical potentials $\mu_{1,2}$ ), device potential $U$ and scattering self-energy $\left[\Sigma_{S}\right]$ go as inputs to NEGF formalism and it provides the transport properties such as carrier density and terminal current.) . . . . . . . . . . . . . .

5.2 (a) Density of states (DOS) and conductance (inset) calculation from atomistic tight binding RGFA for two different widths, (b) Similar calculations over a wider energy range done with KSF, an integration of the $E-k$ dispersion and from analytical expression in Ref. [3]. Conductance from KSF (red line, inset) matches with simple linear approximation of no. of modes (black circles) at low energy. . . . . . . . . . . . . . . . . . . .

5.3 Ballistic NEGF calculation of total conductance $G$ of single layer graphene and bilayer graphene reveals electron hole asymmetry and Fabry-Pérot oscillation. The lowest mode in single layer does not show such oscillation but the bilayer does manifesting Klein tunnel (or reflection). . . . . . . . . . . . .

5.4 Device schematic of a double gate structure ( $\left.\mathrm{W}=1 \mu \mathrm{m}, \mathrm{L}=3 \mu \mathrm{m}, L_{t o p}=1 \mu \mathrm{m}\right)$. We show detailed transport behavior, a-b-c) Resistance variation as a function of gate voltages at various temperatures, d) Short channel effects in the device, the boundary potential works as an effective doping and shifts the Dirac point. Decreasing gate oxide thickness increases gate control with peak resistance remaining the same. . . . . . . . . . . . . . . . .

5.5 Left: device schematic to study metal-graphene interaction. Graphene under metal is doped with $\Delta E_{F}$ and the potential at the boundary (middle) between metal and graphene is $V_{B}$, which decays as $\sim 1 / \sqrt{x / l_{s}}, l_{s}$ being the scaling length. Right: zero temperature transfer characteristics for common metals from NEGF calculation of $50 \mathrm{~nm} \times 1 \mu \mathrm{m}$ graphene, $t_{o x}=100 \mathrm{~nm}$. The boundary potential $V_{B}$ plays the important role of deciding electron hole asymmetry, position and value of the peak resistance while $\Delta E_{F}$ determines the lowest achievable resistance. Inset shows the variation of minimum conductivity $\sigma_{\min }$, realistic metals show deviation from the ideal picture at short lengths. . . . .

5.6 Output characteristics of graphene, a) without contact effects showing no saturation due to lack of bandgap, b) with contacts effects (doped contacts) showing negative differential resistance (NDR) . . . . . . . . . .

5.7 Output characteristics of a single graphene $p n$ junction, a) Abrupt junction showing strong NDR, b) Smooth junction with suppressed NDR. . . . . . . .

5.8 Modeling diffusive transport in graphene, a-b) potential landscape for various impurity concentration, c) evolution of minimum conductivity at the Dirac point, d) total conductivity as a function of channel carrier density from ballistic to diffusive. Channel length, $\mathrm{L}$ is $1 \mu \mathrm{m}$ and width, $\mathrm{W}=0.5 \mu \mathrm{m}$. . . 
5.9 (a) In presence of a magnetic field, the current is carried by the edge states that separate into $+k$ states at the upper edge in equilibrium with the left contact and $-k$ states at the lower edge in equilibrium with the right contact. (b) The Hall voltage goes through plateaus at $\frac{4 q^{2}}{h}(N+1 / 2)$ for single layer and $\frac{4 q^{2}}{h}(N+1)$ for bilayer graphene with $N$ a non-negative integer. Note the presence of a jump at zero energy, which is not seen in a two-dimensional free electron gas (and arises from a half-filled Landau level at the Dirac point), and the additional factor of 2 in the plateau heights, arising from valley degeneracy. (c) For a placement of the Fermi energy between two Landau levels, electron distribution function $\mathrm{f}(\mathrm{E})$ at the top and bottom edges resemble Fermi-Dirac distributions of the corresponding contacts. Since each current carrying state sees a constant electrochemical potential (along transport direction $x$ ), the longitudinal resistance vanishes. . . . . . . . . . . . . . . . . . . 72

A.1 Transverse modes indicated by red horizontal lines . . . . . . . . . . . . . 84

A.2 Energy band diagram of a smooth pn junction with the forbidden region . . 86

A.3 Exact analytical vs. approximate closed form expression, Eq. A.47 . . . . . . 88 


\section{Chapter 1}

\section{Introduction}

\subsection{Graphene: fundamentals, potentials and challenges}

The thinnest material in the world - graphene is one atomic layer of graphite, made up of Carbon atoms in honeycomb lattice structure. It is the two-dimensional version of Carbon, which exists in various forms and dimensions (0-D Fullerene, 1-D Carbon nano-tubes, 3D graphite and diamond). Ever since its discovery [4], it has been under intense research efforts for its unique physical properties. Some of the novel phenomena are the photon-like linear $E-K$ dispersion [5], record high mobility at room temperature $[6,7,8]$, extremely high

thermal conductivity [9], highest mechanical strength [10] and high optical transparency [11]. The discovery of graphene not only demonstrated the exceptional properties of graphene, but triggered the discovery of other 2-D materials (such as transitional metal dichalcogenides), with the possibility of new applications.

Below we discuss the fundamental electronic structure of graphene and its strengths and weaknesses when it comes to field effect devices. 

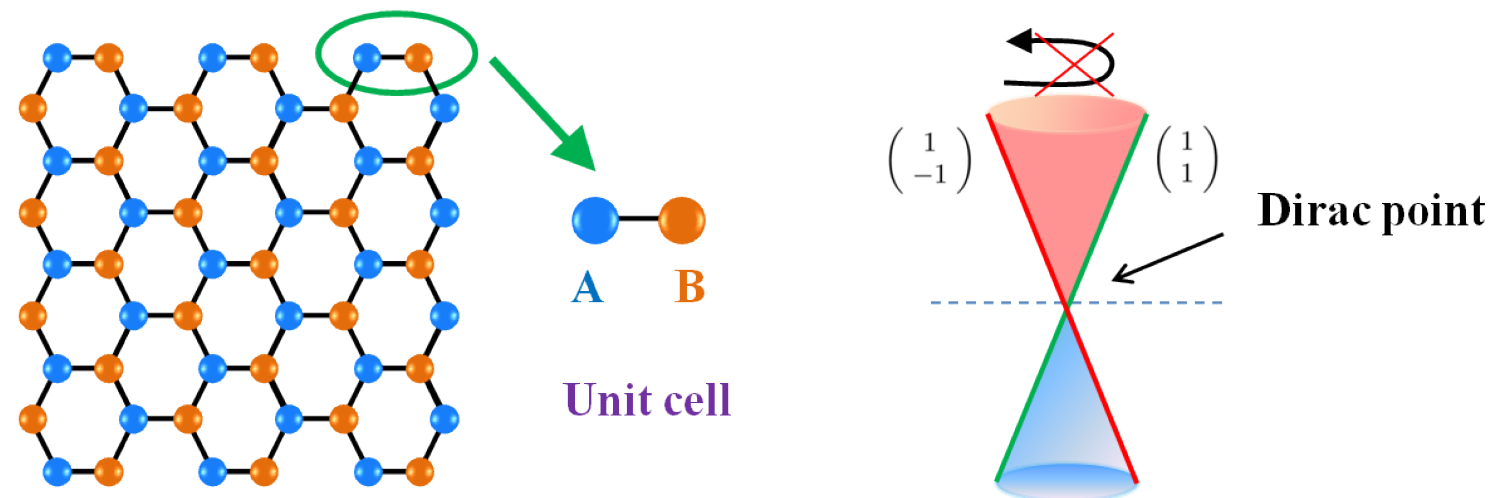

Figure 1.1: Graphene's honeycomb lattice structure. Amplitudes of Bloch functions on the A, B sites mimic two spin systems. They may be called pseudospins, which dictate the electronic properties such as suppressed backscattering.

\subsubsection{Electronic properties of graphene}

Carbon atoms in graphene are arranged in hexagonal lattice structure (Fig. 1.1). The material is composed of two sub-lattices (A-B), each of them following their individual hexagonal arrangement of atoms. If we take two neighboring atoms (one each from A and B), we form the unit cell of graphene (Fig. 1.1). The lattice vectors are

$$
\begin{aligned}
& \overrightarrow{a_{1}}=a \hat{x}+b \hat{y} \\
& \overrightarrow{a_{2}}=a \hat{x}-b \hat{y}
\end{aligned}
$$

where $a=\sqrt{3} a_{c} / 2, b=a_{c} / 2$ and lattice constant, $a_{c}=\sqrt{3} a_{0}=\sqrt{3} \times 1.42=2.46 A^{0}$. Reciprocal space lattice vectors are [12],

$$
\begin{aligned}
& \overrightarrow{A_{1}}=c \hat{x}+d \hat{y} \\
& \overrightarrow{A_{2}}=c \hat{x}-d \hat{y}
\end{aligned}
$$

where $c=(3 / 2) b_{c}, d=\sqrt{3} / 2 b_{c}$ and reciprocal space lattice constant, $b_{c}=4 \pi / 3 a_{0}$. The three high symmetry points are $\Gamma, \mathrm{K}$ and $\mathrm{M}$ which are zone center, the corner and the center of the edge respectively. We will later see that the inflection points in graphene occur at the 

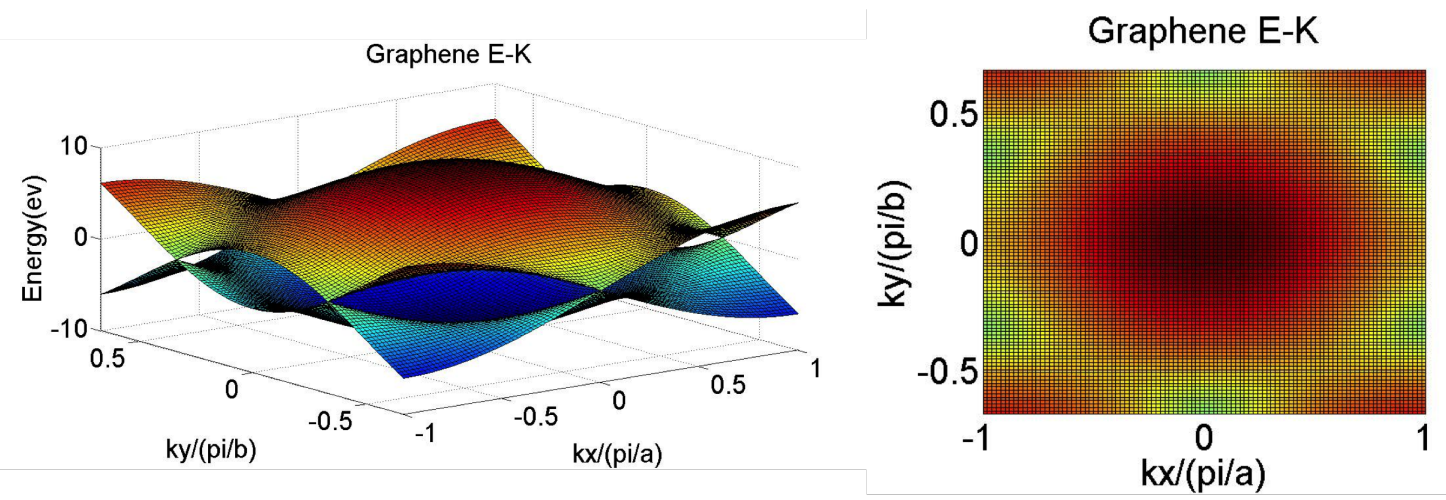

Figure 1.2: Graphene bandstructure

corners of the first Brillouin zone.

Among the valence electrons in graphene, $2 s, 2 p_{x}, 2 p_{y}$ are decoupled from $2 p_{z}$ electrons and form levels that are far from the Fermi energy. The only orbitals that form levels near the Fermi energy are the $2 p_{z}$ orbitals and therefore a one $p_{z}$ orbital basis Hamiltonian is accurate to describe graphene's electronic properties. From the principle of bandstructure, $[h(\vec{k})]=\sum_{m}\left[H_{n m}\right] e^{i \vec{k} \cdot\left(\overrightarrow{d_{m}}-\overrightarrow{d_{n}}\right)}$, we can write,

$$
H(\vec{k})=\left[\begin{array}{cc}
0 & h_{0} \\
h_{0}^{*} & 0
\end{array}\right]
$$

here $h_{0}=-t\left(1+e^{i \vec{k} \cdot \overrightarrow{a_{1}}}+e^{i \vec{k} \cdot \overrightarrow{a_{2}}}\right)$. So the eigenvalues are (shown in Fig. 1.2)

$$
E= \pm t \sqrt{\left(1+4 \cos k_{y} b \cos k_{x} a+4 \cos ^{2} k_{y} b\right)}
$$

where the Carbon-Carbon tight binding parameter, $t$ is $-2.7 \mathrm{~V}$. At low energy (near the Fermi energy) the $E-K$ is approximated as $\left(v_{F}=\frac{1}{\hbar} \frac{\delta E}{\delta K}=\frac{1}{\hbar} \frac{3 t a_{c c}}{2}\right)$,

$$
E= \pm \hbar v_{F} k
$$




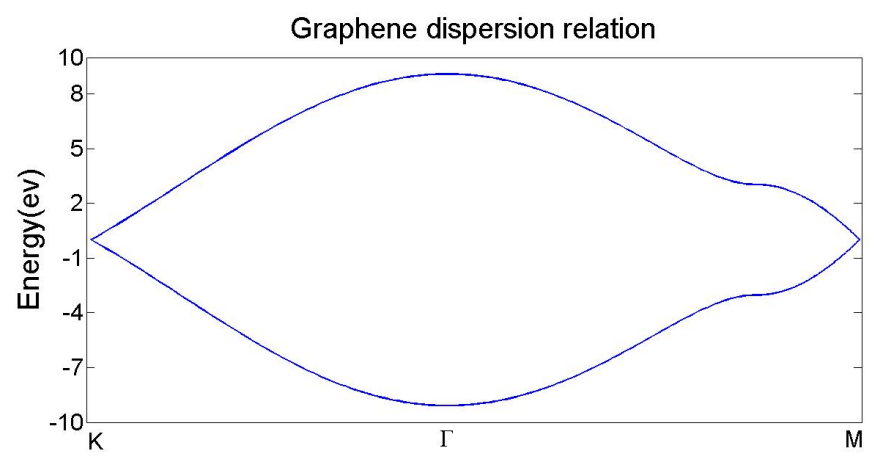

Figure 1.3: Dispersion of graphene along different directions. There is no bandgap and $E-K$ is linear around the $K$ point.

from the simplified Hamiltonian,

$$
H(\vec{k})=\frac{3 t a_{c c}}{2}\left[\begin{array}{cc}
0 & k_{x}-i k_{y} \\
k_{x}+i k_{y}^{*} & 0
\end{array}\right]
$$

which can be written as -

$$
\begin{array}{r}
H(k)=\hbar v_{F} \vec{\sigma} \cdot \vec{k} \\
H(k)=v_{F} \vec{\sigma} \cdot \vec{p}
\end{array}
$$

where $\sigma_{x}, \sigma_{y}$ etc. are the Pauli matrices. The simplification (after Taylor expansion) is valid around the inflection points.

Eigenvectors of graphene: We can do more simplification

$$
H(\vec{k})=\hbar v_{F}|k|\left[\begin{array}{cc}
0 & e^{-i \theta} \\
e^{i \theta} & 0
\end{array}\right]
$$

From this, the eigenvectors are

$$
\psi=\left[\begin{array}{c}
1 \\
e^{i \theta}
\end{array}\right]
$$


and

$$
\psi=\left[\begin{array}{c}
1 \\
-e^{i \theta}
\end{array}\right]
$$

The real space eigenvectors can be written as

$$
\Psi^{k}(\vec{r})=e^{i \vec{k} \cdot \vec{r}}\left[\begin{array}{c}
1 \\
s e^{i \theta}
\end{array}\right]
$$

where $\mathrm{s}=\operatorname{sign}(E)$ and $\theta=\tan ^{-1} \frac{k_{y}}{k_{x}}$. Thus the wavefunction has two components corresponding to two sub-lattices (A-B in Fig. 1.1) in graphene crystal. The two component wavefunction mimics a two spin system and is known as pseudospin degree of freedom for graphene. The pseudospins play an important role in graphene transport. The backscattering probability (from Fermi's golden rule) which is proportional to $\left|\psi_{i}\right| V\left|\psi_{f}\right|^{2}$ is suppressed due to the orthogonality between the forward ([1 1]) and backward ([1 -1]) moving branches resulting in low scattering rate $(1 / \tau)$. The other remarkable feature of graphene bandstructure is the linear dispersion near the Brillouin zone corners [5]. This leads to very low effective mass $\left(m^{*}\right)$. Both these factors $\left(1 / \tau\right.$ and $\left.m^{*}\right)$ contribute to very high carrier mobility $(\mu)$, which can be near $200,000 \mathrm{~cm}^{2} / \mathrm{Vs}[6,7]$ at room temperature for suspended devices compared to $\sim 1000 \mathrm{~cm}^{2} / \mathrm{Vs}$ in silicon. But the linear E-K comes with zero bandgap with the Fermi level lying at the position the conduction and valence bands meet (Fig. 1.2, 1.3). Owing to this semi-metalic nature (Fig. 1.3), the current modulation with gate voltage is modest $(\sim 5-20)$ compared to $10^{5}-10^{6}$ for a semiconductor with a sizable bandgap. We next discuss briefly how graphene based field-effect transistors behave based on such electronic properties.

\subsubsection{Silicon CMOS and graphene based transistors}

Fig. 1.4 shows the current-voltage characteristics of CMOS technology relative to graphene and the desired behavior. One of the greatest problems in the current semiconductor industry 


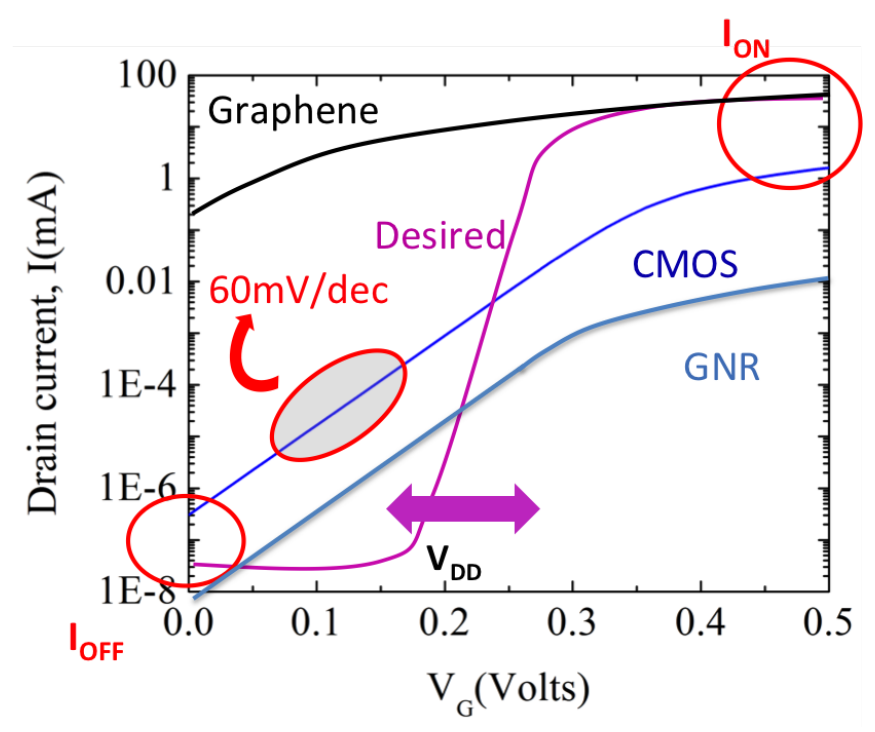

Figure 1.4: Current voltage characteristics of CMOS and other technologies. Unless the transition rate $(60 \mathrm{mV} / \mathrm{Dec})$ of current is changed (desired $I_{D^{-}} V_{G}$ in purple), power dissipation $\left(0.5 C V_{D}^{2} f\right)$ and operating frequency $(f)$ cannot be improved.

is the trade off between performance and heat generation. A high frequency $(f)$ chip is desired for high performance but it increases the dynamic power dissipation, $P=\frac{1}{2} C V_{D D}^{2} f$, where $C$ is the gate capacitance and $V_{D D}$ is the supply voltage typically required to make the transition from $\mathrm{OFF}$ to $\mathrm{ON}$ state. A high $\mathrm{ON}-\mathrm{OFF}$ current ratio is desired for reliability (typically $\sim 10^{6}$ ) and therefore the slope of current-voltage determines the required $V_{D D}$. The reduction of power dissipation requires a low $V_{D D}$. For CMOS, the slope is set by the thermionic emission principle for electrons over a barrier and cannot be reduced unless a different technology is used. In addition to the above requirements, a high ON current $I_{O N}$ is desired to have high drive capability. Therefore the purple line in the Fig. 1.4 shows the desired transfer curve with steeper transition, high ON current and low OFF current. The black line shows the transfer curve for graphene, closely matching the desired $I_{O N}(\sim$ $10 \mathrm{~mA} / \mu \mathrm{m}$, more than 10 times than Silicon CMOS) but fails to meet the low OFF current requirement.

There have been efforts to open bandgap in graphene to increase the ON-OFF ratio through various means (e.g. quantizing the sheet into nanoribbons [13, 14, 15], applying 

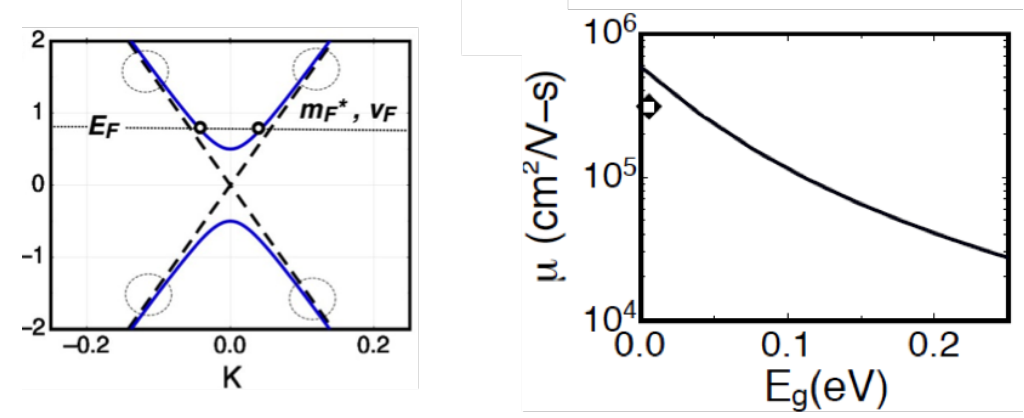

Figure 1.5: Mobility degradation as bandgap is opened in graphene (figure from Frank Tseng).

strain [16] or electric field in bilayer graphene [17]), but as soon as we open the bandgap, the linearity of the bandstructure is distorted leading to parabolic dispersion and massive electrons (Fig. 1.5). Thus intrinsically the mobility degrades and we lose the advantage of graphene's high mobility [18, 19]. Also there are practical limitations with graphene nanoribbons (GNR), such as difficulty in building narrow ribbons $(<10 \mathrm{~nm})$ with smooth (without roughness) edges.

On the other hand, the transistors for radio frequency $(\mathrm{RF})$ technology does not require high current ON-OFF ratio [20]. The desired figures of merit are current and voltage gain, good current saturation $\left(I_{D}\right.$ vs $\left.V_{D}\right)$, high ON current, high cut-off frequency and device scalability. Graphene's high mobility makes the ON current impressive (in $\mathrm{mA} / \mu \mathrm{m}$ regime for ballistic devices). The high carrier velocity and high mobility yields high cut off frequency (300-400 GHz, [21, 22]). Also, good small signal gain and current saturation have been achieved for devices up to hundreds of nanometer long [23, 24]. The challenge now lies in designing and building devices with good gate control and minimum DIBL (drain induced barrier lowering) for short channel devices (<100 nm) [25, 26].

\subsubsection{How close are we to realizing ballistic transport?}

Many of the exceptional properties of graphene and the properties that are manipulated and predicted in this work require ballistic transport. Graphene device fabrication techniques have progressed rapidly since its discovery and the quality of graphene devices is increasing 
to approach the ballistic limit. Extremely long mean free path (up to $\mu \mathrm{m}$ ) has already been achieved in a number of recent experiments [8, 27, 28, 29, 30]. As a result, ballistic quantum transport phenomena such as quantum interference pattern due to formation of Fabry-Pérot cavity has been demonstrated [31, 27, 28].

There are three main techniques to grow graphene crystal: mechanical exfoliation, epitaxial graphene (on similar lattice structure such as $\mathrm{SiC}$ ) and chemical vapor deposition on a metal (such as $\mathrm{Ni}, \mathrm{Cu}$ ). The mechanical exfoliation method was used in the seminal work of Novoselov et. al. [4], where one layer graphene was separated from graphite by repetitive peeling of graphene layers.

In 2008, Yu et. al. [32] showed a chemical vapor deposition technique, which can produce large scale high quality graphene crystal on metal substrates $(\mathrm{Ni} / \mathrm{Cu})$ followed by cooling and transfer onto an insulating substrate of choice. The choice of substrate has proved to be crucial in graphene electron transport properties. Graphene on $\mathrm{SiO}_{2}$ substrate has been studied extensively and in most cases the mobility is limited to $2000-4000 \mathrm{~cm}^{2} / \mathrm{V}-\mathrm{s}[33,2]$ due to the presence of impurity scattering and phonons. Dean et. al.[30] showed in 2010 that hexagonal Boron-Nitride (hBN) is an excellent substrate for graphene. $\mathrm{hBN}$ is an insulator with a similar lattice constant as graphene. It is relatively free from trapped charges due to its inert nature and the surface optical phonons are at higher energies compared to $\mathrm{SiO}_{2}$. Due to the combination of these factors, graphene on hBN has extremely high mobility (more than $100,000 \mathrm{~cm}^{2} / \mathrm{V}$-s at low carrier density and $25,000 \mathrm{~cm}^{2} / \mathrm{V}$-s at high carrier density). Due to its excellent dielectric properties, hBN-graphene-hBN heterostructure are fabricated as an oxide-channel-substrate combination [8]. Using the combination of CVD grown graphene and hBN substrate, it is shown that graphene mobility can easily surpass any other material $[29,8]$.

Using suspended graphene, it is possible to eliminate substrate induced scattering altogether and mobility close to graphene's intrinsic mobility can be achieved. Suspended graphene devices were used recently to show ballistic quantum interference [28, 27]. 
One major obstacle toward graphene based nanoelectronics is the contact resistance between metal and graphene. In most cases, the metal-graphene contact is weak, which creates distortion of the graphene bandstructure (graphene under metal for surface contacts) leading to high contact resistance $(\sim 500-1000 \Omega-\mu \mathrm{m})$. It has been shown recently that side edge contacts as opposed to surface contacts achieve better overlap between orbitals leading to substantially lower contact resistance [8]. The extracted mobility exceeded 100,000 $\mathrm{cm}^{2} / \mathrm{V}-\mathrm{s}$.

\subsection{Controlling electron flow in graphene $p n$ junction}

This research is primarily focused on graphene pn junction (GPNJ), built with gate voltage. The GPNJ has unique electronic properties, among which the analogy with optics and incident angle dependent tunneling are the two most intriguing phenomena. It has been theoretically shown $[34,35]$ that the gate voltage plays the role of refractive index for carriers in graphene and the pn junction interface works as a focusing lens. Owing to the lack of bandgap, the conduction and valence bands cross easily and carriers tunnel from conduction band to valence band or vice versa. Due to the change in carrier velocity in the two bands, they bend at the interface to focus at one point for equal but bipolar dopings. This is analogous to what is known as a Veselago lens in optics with negative refractive index metamaterials [36] and comes with its own Snell's law. Such eletron trajectory is accompanied with graphene's equivalent Fresnel's equations, which dictate the transmission probability depending upon the incident angle of the carrier [37, 2]. Compared to uniformly doped graphene, we have higher control over the conductance in GPNJ with gate voltage [38]. Another unique attribute of GPNJ is that for normal incidence (the transverse mode with $k_{y}=0$ ), the transmission is always unity, regardless of voltage gradient across the junction. From a device point of view, this particular phenomenon (known as Klein tunneling) [37, 39], further limits current modulation with gate voltage. 

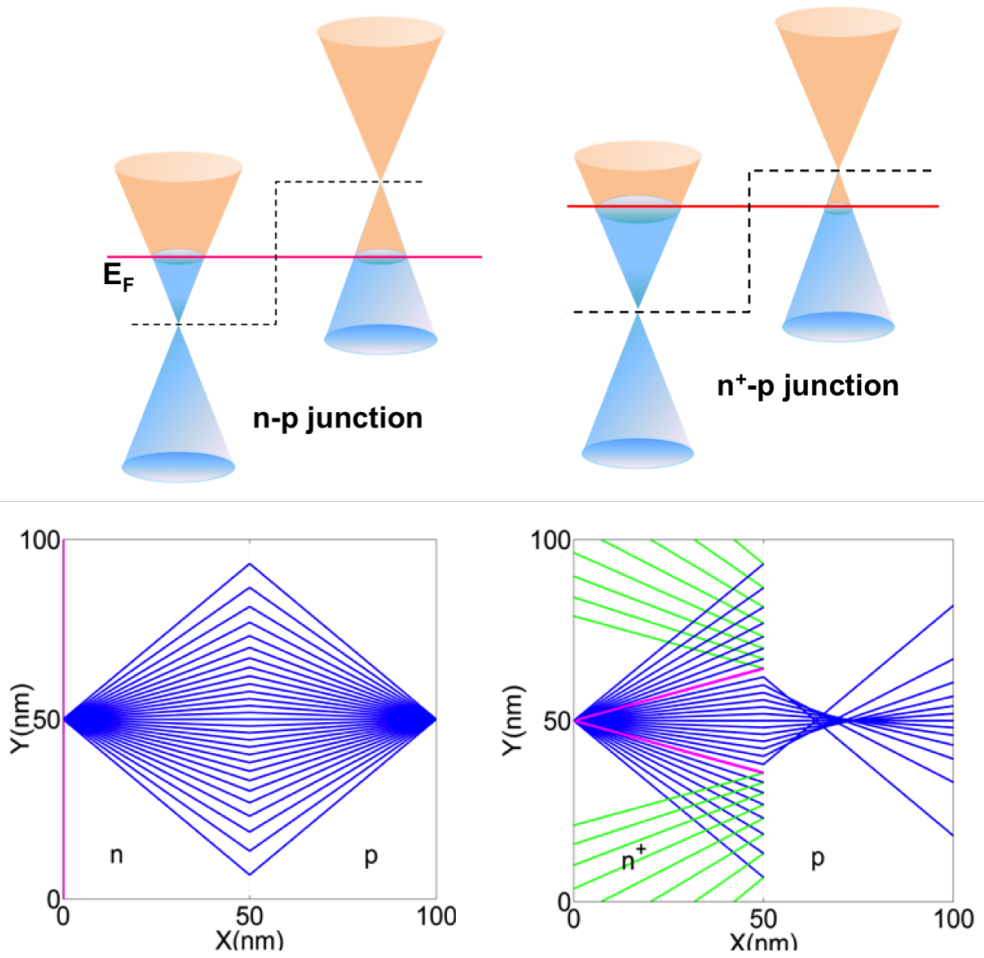

Figure 1.6: Electron 'optics' at a graphene pn junction. Electron trajectories (below) mimic optical refraction and depend on electrostatic doping levels (above).

Fig. 1.6 shows the electron focusing trajectories on the surface of a GPNJ, doped symmetrically $\left(V_{G 1}=-V_{G 2}\right)$. With asymmetric doping, $\left(\left|V_{G 1}\right|>\left|V_{G 2}\right|\right)$, carrier reflect back above a critical angle, exhibiting total internal reflection. Such control begs a question whether we can sufficiently control the carrier flow to the extent of turning OFF the device and achieve a transistor action. If it is indeed possible to do that, it would come without the expense of opening a bandgap in graphene. It is the purpose of this dissertation to show how such switching can be achievable, along with its limitations and possible applications. In addition to analytical quantum mechanical (QM) treatment of the problem, we simulate such devices with a sophisticated numerical formalism called Non-Equilibrium Green's function (NEGF), which is commonly used to simulate quantum devices. The numerical model is used to show simulation results of a number of graphene experiments. 


\subsection{Overview of the dissertation}

The dissertation is primarily divided in four parts.

- Chapter 2 describes the physics of carrier tunneling in graphene pn junction and how the angle dependent transmission through GPNJ can be tuned with gate parameters. We report benchmarking with recent experimental results that provide evidence of such control.

- Chapter 3 provides device designs that produce transmission gap. For the first time, we show how a material without a bandgap can switch and produce high ON-OFF ratio. The transmission gap relies on chiral tunneling and does not distort graphene's linear dispersion. We show that such a gap is determined by the gate voltage gradients across the junction and can be collapsed by going from heterogeneous to homogeneous doping limit, producing an intriguing low inverse sub-threshold slope in the device transfer characteristics.

- In Chapter 4, we discuss how the GPNJ based switching idea can be useful compared to CMOS. We also show how the use of pn junction in topological insulators (TI), a material with very similar bandstructure as graphene on the surface, can produce spin polarized current by using the same physics as graphene.

- Along with analytical results, both Chapters 2 and 3 contain results using Non-Equilibrium Green's function (NEGF) formalism based numerical simulation results. In Chapter 5, we formally discuss the basics of the formalism. The computational platform is optimized to simulate graphene- like systems in both ballistic and diffusive regime to capture a number of recent experiments.

We then draw conclusions with scope for future research. We include several appendices (A-C) to describe the detailed derivation of the analytical expressions, required to understand the transmission, conductance properties of GPNJ and spin filtering in TI. 


\section{Chapter 2}

\section{Transport physics of graphene pn junction}

\subsection{Angle dependent tunneling in GPNJ}

\subsubsection{Carrier trajectories: Snell's law for graphene}

pn junctions in graphene can be built electrostatically, as shown in Fig. 2.1. Graphene bands (colored cones) are shifted up or down depending on the gate voltage sign. The red line shows the Fermi energy. Since there is no bandgap, carriers directly tunnel from conduction band ( $\mathrm{CB}$ on left) to valence band (VB on right). An electron at state $\left(k_{x}, k_{y}\right)$ in $\mathrm{CB}(n$ side, left) injected at the Fermi level $\left(E_{F}\right)$ tunnels from left to right at the VB ( $p$ side, right) as illustrated in Fig. 2.2a. The Fermi surface from the top at the indicated $E_{F}$ is shown in Fig. 2.2. Since there is no electric field applied along $y$, the transverse momentum $k_{y}$ is conserved. In the $p$ side, both states $\left(k_{x}, k_{y}\right),\left(-k_{x}, k_{y}\right)$ conserve $k_{y}$ but only the $\left(-k_{x}, k_{y}\right)$ state conserves the direction of the velocity $v_{x}$ along $x$. This is due to the fact that the carrier

group velocity (defined as $v=\frac{1}{\hbar} \frac{\delta E}{\delta k}$ ) changes sign from CB to VB. A group of electrons thus injected from a point source, spatially bend at the $p n$ interface and focus at one point (Fig. 2.2a), as predicted in Ref. [34]. This is analogous to the Veselago lens in optics with negative 


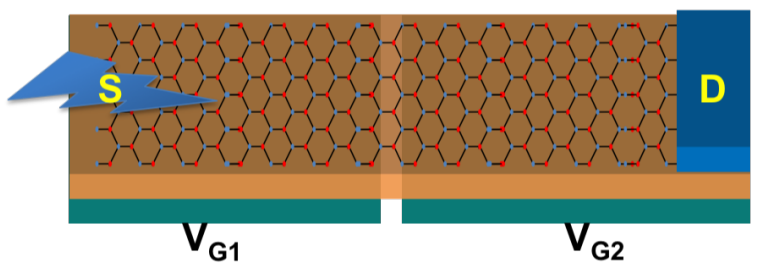

Figure 2.1: Schematic of a graphene based $p n$ junction device with a point contact (much smaller than the width of the device) as an injector and a wide drain to collect the electrons. Electrostatic doping shifts graphene bands up/down depending on voltage sign. Bands cross at the Fermi energy, shown in red, enabling band to band tunneling.

refractive index metamaterial [36]. The conservation of $k_{y}$ yields the equivalent Snell's law for graphene,

$$
k_{F 1} \sin \theta_{1}=-k_{F 2} \sin \theta_{2}
$$

where $k_{F 1}$ and $k_{F 1}$ are total wave-vectors on both sides. For equal dopings, the focusing is perfect (at zero temperature). For unequal doping such as, $n^{+} p$, electrons above a critical angle reflect back (total internal reflection (TIR)), as shown in Fig. 2.2b, because they are unable to conserve $k_{y}$. This can be easily understood from the unequal radii of the Fermi surfaces.

$$
\theta_{C}=\sin ^{-1} \frac{k_{F 2}}{k_{F 1}}
$$

By angles, we simply mean the angular representation of various transverse modes $(\theta=$ $\left.\tan ^{-1}\left(\frac{k_{y}}{k_{x}}\right)\right)$ available at a particular doping level, $E_{F}$ (Fig. 2.5).

We perform an NEGF simulation (discussed in detailed in Chapter 5) of the device shown in Fig. 2.1 to show the electron trajectories inside the GPNJ device. The total current at 

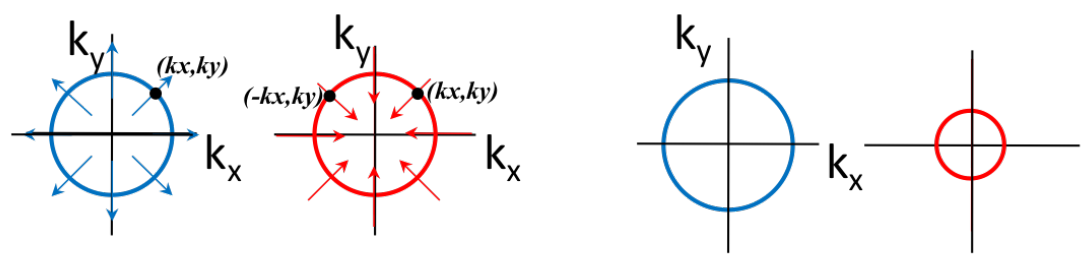

\section{Conduction band Valence band}
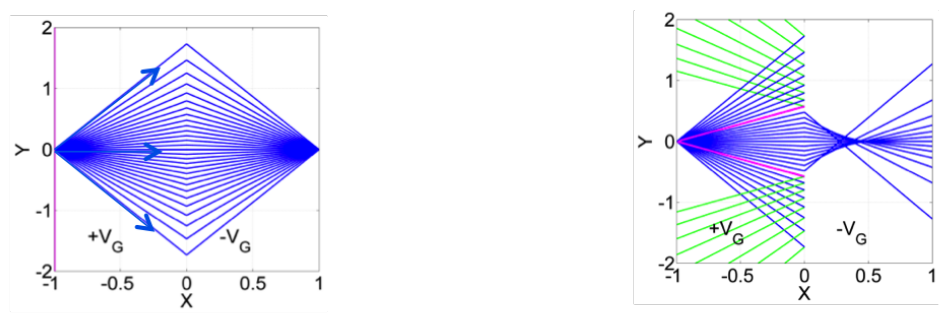

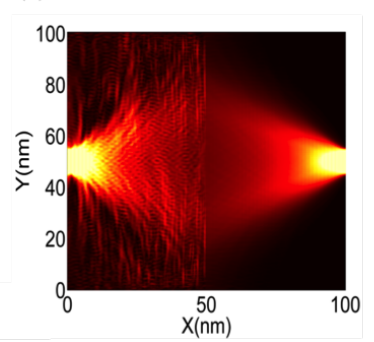

a) Focusing

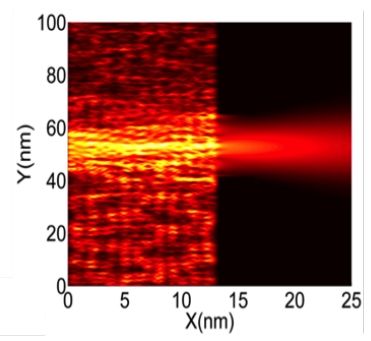

b)Total internal reflection

Figure 2.2: Carrier trajectories in GPNJ, a) symmetric doping and focusing of electron flow, b) asymmetric doping where the refracted side has lower doping (smaller Fermi surface), electrons reflect back above a critical angle.

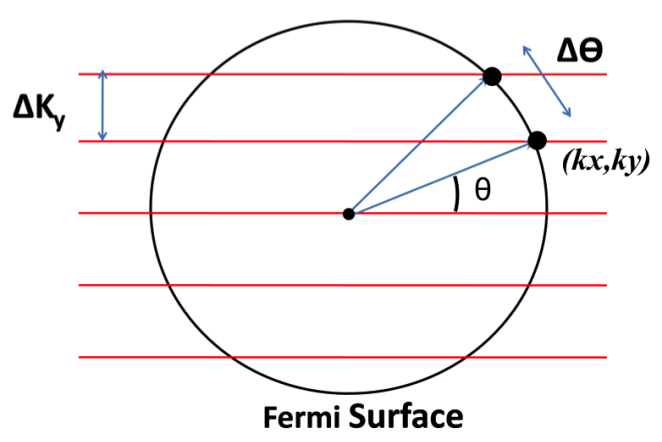

Figure 2.3: Definition of $\theta$ in $k$ space.

an atomic site is equal to the sum of all current components flowing to nearest neighbor atoms. For equal dopings on both sides $\left(k_{F 2}=k_{F 1}\right)$, the angle of refraction is exactly equal 
(with a negative sign) to the incident angle. As a result, a group of electrons originating from a point contact focus back to one point on the refracted side [34]. Fig. 2.2b shows the trajectories when the doping at the refracted side is smaller than that at the incident side $\left(k_{F 2}<k_{F 1}\right)$ making the critical angle smaller than $\pi / 2$. The geometrical 'optics' trajectories corresponding to electron focusing and total internal reflection and NEGF simulation results agree quite well. A small source contact (10 nm wide) is placed $50 \mathrm{~nm}$ to the left of the $p n$ junction, and electrons are injected with a small drain bias $\left(V_{D}=0.08 \mathrm{~V}\right)$ around a Fermi energy. When the gates are biased symmetrically around the junction, although the electrons see a voltage bias along the drain that spans the entire device width, the pn junction Hamiltonian and associated pseudospin conservation causes the electrons to focus to a small point at the drain. For an $n^{+} p$ junction, the electrons incident above the critical angle are unable to preserve their transverse quasi-momentum and reflect back, while those within critical angle tunnel to the other side.

\subsubsection{Transmission probability: Fresnel's equation for graphene}

The graphene wavefunction has the form of a spinor

$$
\Psi=\left[\begin{array}{l}
\psi_{1} \\
\psi_{2}
\end{array}\right]
$$

Matching these two components on both sides of the junction (Fig. 2.4) gives the transmission probability. The transmission probability thus depends on how well the pseudospins match on both sides of the junction. We get (details are in Appendix A),

$$
T(\theta)=\frac{\cos \theta_{1} \cos \theta_{2}}{\cos ^{2}\left(\frac{\theta_{1}+\theta_{2}}{2}\right)}
$$

where $\theta_{1}$ is the incident angle and $\theta_{2}$ is the refracted angle connected by Snell's Law, Eq. 2.1. This is a general equation [40] for arbitrary $E_{F}$ and gate voltage gradient $V_{0}$. The polar 


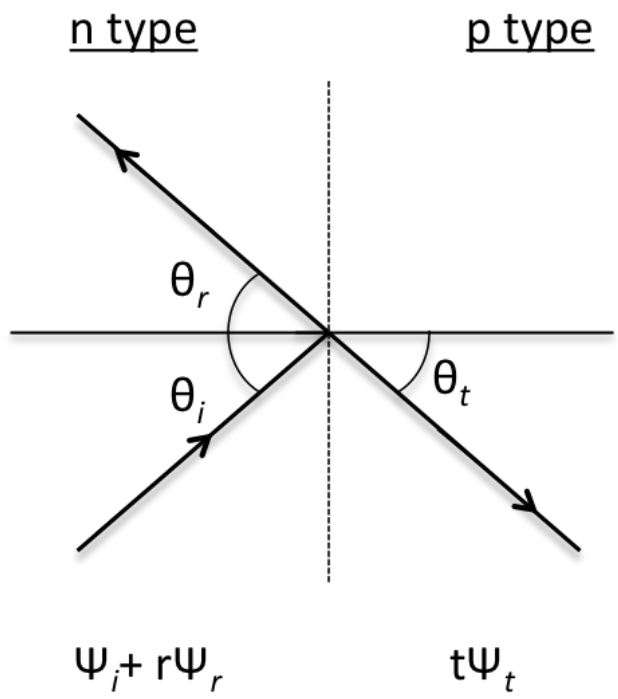

Figure 2.4: Matching wavefunctions on both sides gives transmission probability

plot in Fig. 2.5 shows the incident angle dependence of transmission at a given $E_{F}$. One remarkable fact is that for normal incidence $\left(\theta_{1}=0\right)$, the transmission is unity, regardless of $V_{0}$. This is known as Klein tunneling $[37,41]$ reminiscent of the Klein paradox in relativistic physics. Such perfect transmission is possible because the wavefunctions on both sides match exactly for normal incidence [1 1 , and mismatch with the backward moving branch [1 -1], leading to complete suppression of backscattering. The higher angles show decreasing transmission. Such angle dependent transmission is possible because the carriers in graphene are chiral, meaning the pseudospin components are related to the direction of momentum $(\theta)$. From angle (transverse mode) dependent transmission, we need to calculate the total conductance, which can be found from,

$$
G\left(E_{F}\right)=G_{0} \sum_{\theta_{1}} T\left(\theta_{1}\right)=G_{0} M\left(E_{F}\right) T_{a v}\left(E_{F}\right)
$$

where $G_{0}=4 q^{2} / h$. A notable point here is that only the total conductance (Eq. 2.4) is a measurable quantity whereas the mode resolved transmission (Eq. 2.3) is extremely difficult to measure. Thus conductance can be written as the number of transverse modes $M\left(E_{F}\right)$ in one side of the graphene sheet times an average transmission of modes $T_{a v}$ to the other side. 

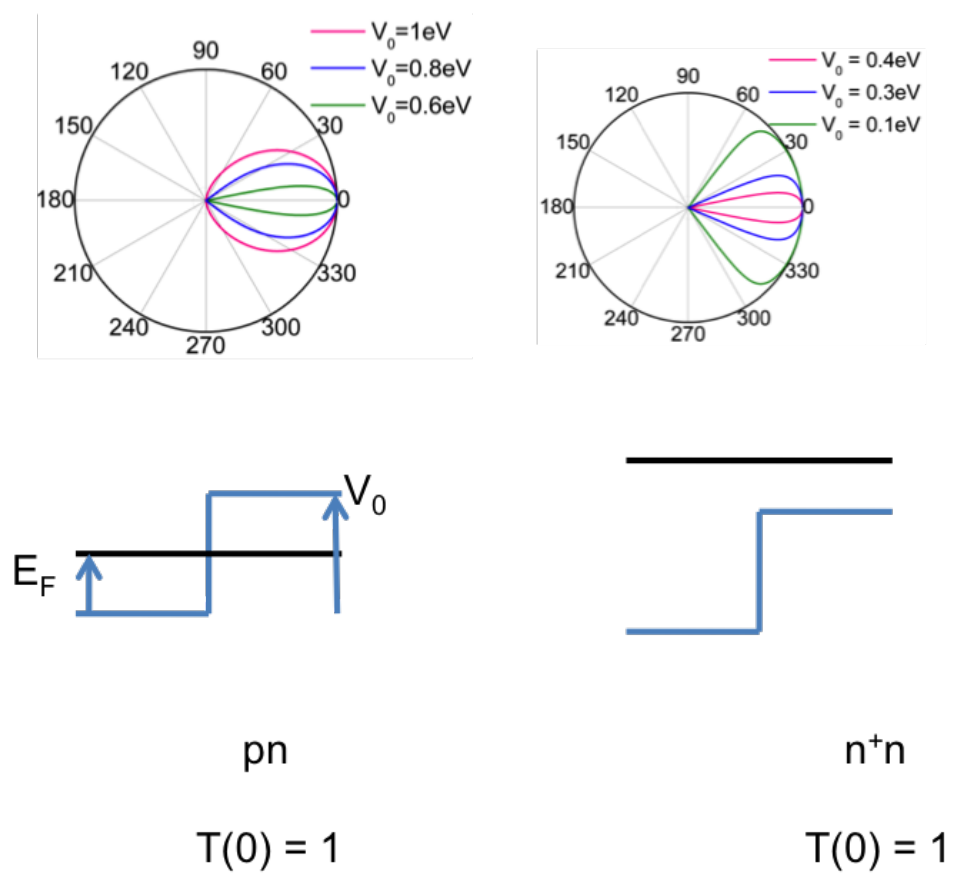

Figure 2.5: Angle dependent transmission for various bias conditions.

For instance, a symmetric GPNJ (at $E_{F}=0$ ) has,

$$
\begin{aligned}
G / G_{0} & =\sum_{\theta} T(\theta) \approx \int_{-\pi / 2}^{\pi / 2} \frac{T(\theta)}{\Delta \theta} d \theta \\
& =\int_{-\pi / 2}^{\pi / 2} \frac{\cos ^{2} \theta}{\Delta k_{y}} k_{F} \cos \theta d \theta=\frac{W k_{F}}{2 \pi} \int_{-\pi / 2}^{\pi / 2} \cos ^{3} \theta d \theta \\
& =\frac{2}{3} M(E)
\end{aligned}
$$

$\Delta \theta=\Delta k_{y} /\left(k_{F} \cos \theta\right)$ is the angular separation between adjacent modes. From above, we get $T_{a v}=2 / 3$, meaning a symmetric GPNJ will have $2 / 3$ times less conductance compared to a uniformly doped graphene sheet. One of the aims of this dissertation is to decrease this $T_{a v}$ many times so that we have a larger modulation of $G$. We will discuss how to do that in the following chapters. Fig. 2.6 shows the total conductance $(G)$ and average transmission per mode $\left(T_{a v}\right)$ as a function of Fermi energy, $E_{F}$, for a fixed built in potential $V_{0}$ for both abrupt and smooth pn junctions. We discuss the smooth junction in the next section.

An npn junction can be formed by either a combination of global back gate and top gate 

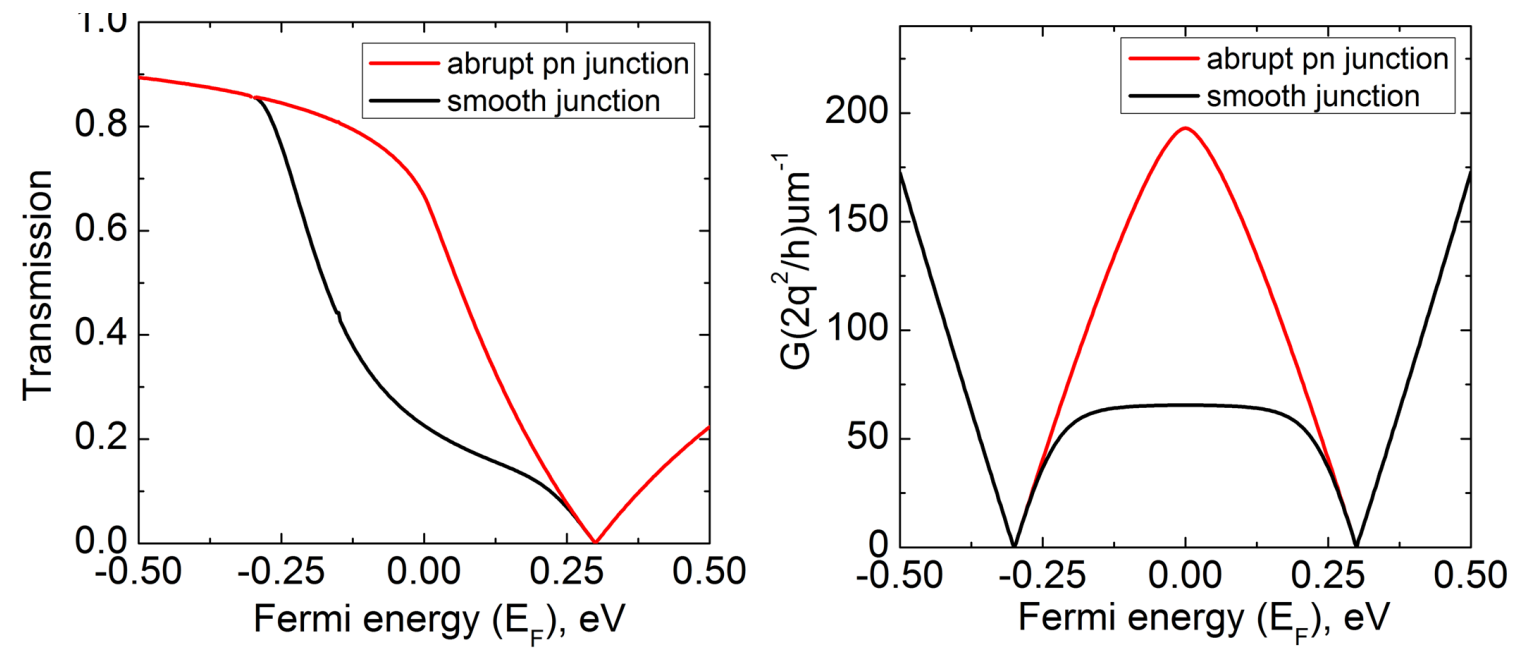

Figure 2.6: a) Average transmission per mode $T_{a v}$ and b) total conductance of GPNJ as a function of Fermi energy $E_{F}$. There are two zeros in the conductance plot signifying the two Dirac points in the system.

[42] or by selective chemical doping. In Fig. 2.7a, we show the energy band diagram for the device. We consider both junctions as abrupt, i.e. $d=0$, while the extent of the $p$ type barrier region is $D$. In Fig. 2.7c, we show an NEGF calculation of the $n p n$ conductance for various $D$ values. For larger $D$, two Dirac points are evident from the pinched off conductance, but for smaller $D$ 's we see a considerable tunneling near the second Dirac point $\left(E_{F}=0.5 \mathrm{eV}\right)$, where the critical angle for the incident electrons is supposed to be very small. The first dip happens at the Dirac point of the incident $n$ region where $M_{1}=0$ as before, while the second corresponds to the carriers with the Dirac point of the barrier $p$ region and the transmission $T_{12}$ is small. In a single $p n$ junction, the modes with higher angles than the critical angle reflect back as they do not have propagating states to tunnel into while preserving their transverse quasi-momentum. But in the npn junction case, the length of the forbidden region $D$ is finite, and the electrons even while aligned with the Dirac point of the central $p$ region can still preserve their quasi-momenta by tunneling to the other side. This increased conductance has been seen experimentally [1] in the past (Fig. 2.7). In the limit when $D$ is very large, we approach the single $p n$ junction case . This is shown in Fig. 2.10c inset. When $D$ is very small, we approach the uniform doping ( $n$ doped here) case through significant 

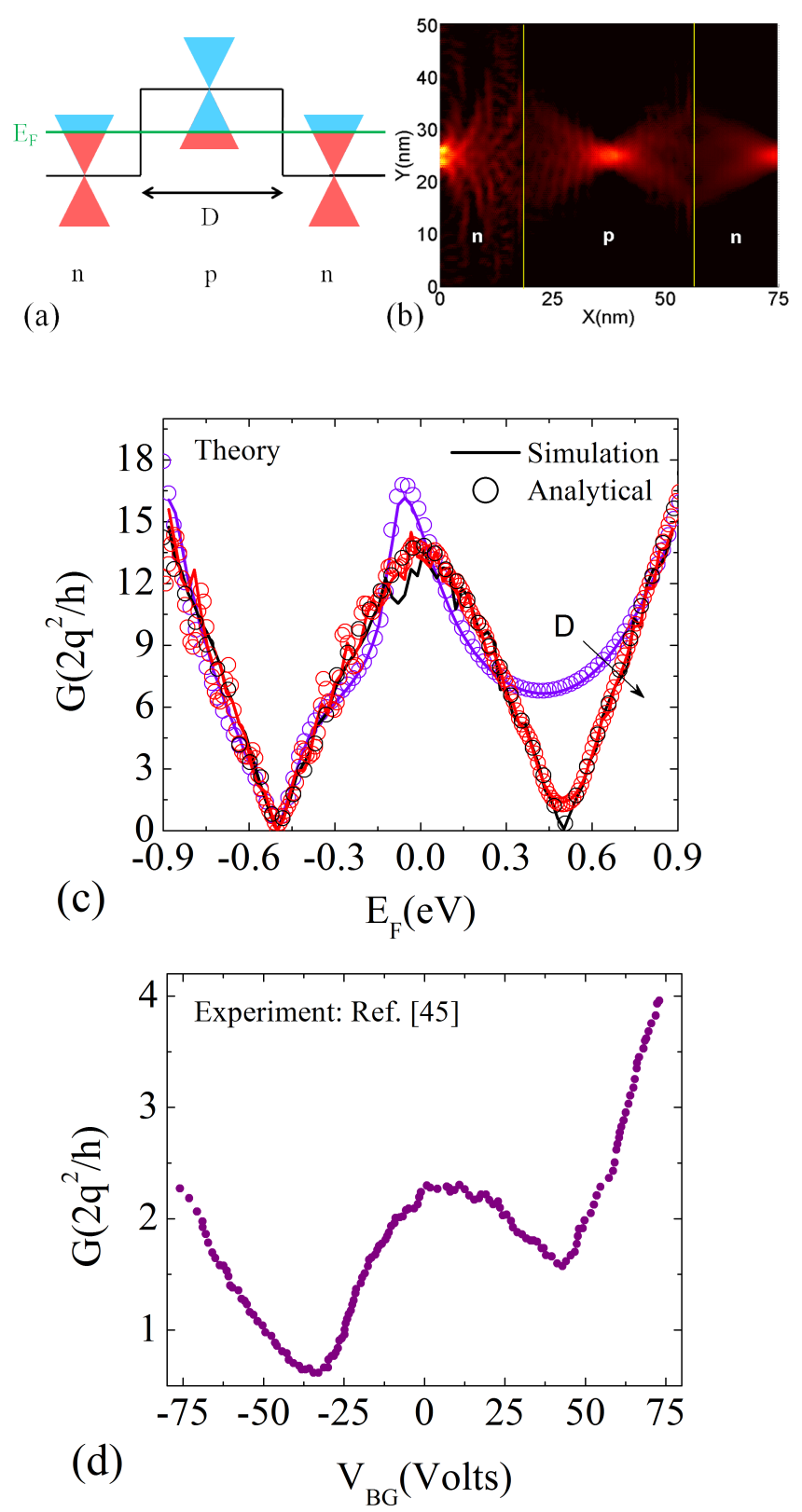

Figure 2.7: Physics of npn junction. (a) Band diagram. The black line shows the change of Dirac point as a result of different dopings at different portions of the device. Blue (red) region in the E-k indicates empty (filled) states. (b) Electron trajectories in such junctions, multiple focusing takes place, (c) Ballistic conductance of a 50nm wide graphene sheet as a function of Fermi energy $\left(E_{F}\right)$ for a fixed barrier height $\left(V_{0}=1 \mathrm{eV}\right)$ but different barrier width, $D=5,25$ and 100nm (violet, red and black). The solid lines are from numerical NEGF calculation, while the circles are analytical calculation from Eq. 2.6, summed over all available modes. (d) Experimental data from Ref. [1]. Conductance in the experiment is much lower due to scattering processes. 
tunneling. Note that we also see oscillations for npn junctions, which originate from the Fabry-Pérot cavity formed by the electrostatic barrier [1, 41]. Both the tunneling and the resonant oscillations can be captured analytically by matching eigenvector components across the npn junction, as worked out in Ref. [37].

$$
r=\frac{2 i e^{i \phi} \sin \left(q_{x} D\right) \times\left(\sin \phi-s s^{\prime} \sin \theta\right)}{s s^{\prime}\left[e^{-i q_{x} D} \cos (\phi+\theta)+e^{i q_{x} D} \cos (\phi-\theta)\right]-2 i \sin \left(q_{x} D\right)}
$$

giving a transmission, $T(\phi)=1-R=1-|r|^{2} . q_{x}$ is the wave vector inside the barrier,

$q_{x}=\sqrt{\left(E_{F}-V_{0}\right)^{2} /\left(\hbar^{2} v_{F}^{2}\right)-k_{y}^{2}}, \theta=\tan ^{-1}\left(k_{y} / q_{x}\right)$ and $s=\operatorname{sign}\left(E_{F}\right)$ and $s^{\prime}=\operatorname{sign}\left(E_{F}-V_{0}\right)$. We then sum over different modes, set by the width of the graphene sheet, to get the total conductance $G\left(E_{F}\right)$ in units of $G_{0}=4 q^{2} / h$ (Fig. 2.7c, circles), in excellent agreement with the NEGF atomistic calculation and qualitatively with experiments (Fig. 2.7d).

\subsection{Controlling GPNJ conductance with gate parame- ters}

\subsubsection{Conductance of smooth $p n$ junction: low pass filter}

A split $d^{1}$ between the two gates, results in a smooth potential variation $U(x)$ across the junction (Fig. 2.8a). It has been shown [43] that such potential profile works as a low pass filter (with respect to angle), suppressing the high angle transmission across the junction exponentially. Since $k_{y}$ across the junction is conserved, the varying potential makes $k_{x}=\sqrt{\left[\left(E-U(x) / \hbar v_{F}\right]^{2}-k_{y}^{2}\right.}$ evanescent over a certain length and therefore it works as a tunnel barrier for electrons with nonzero $k_{y}$. From WKB (Wentzel-Kramers-Brillouin) formalism, the transmission can be written as,

$$
T(\theta)=\frac{\cos \theta_{1} \cos \theta_{2}}{\cos ^{2}\left(\frac{\theta_{1}+\theta_{2}}{2}\right)} e^{-\pi \frac{k_{F 1} k_{F 2}}{k_{F 1}+k_{F 2}} d \sin ^{2} \theta}
$$

\footnotetext{
${ }^{1}$ In the following chapters, we assume gate split $2 d$ to make transmission expressions more compact
} 
This results a sharper lobe in $T(\theta)$ as shown in Fig. 2.8. But normal incidence again shows a
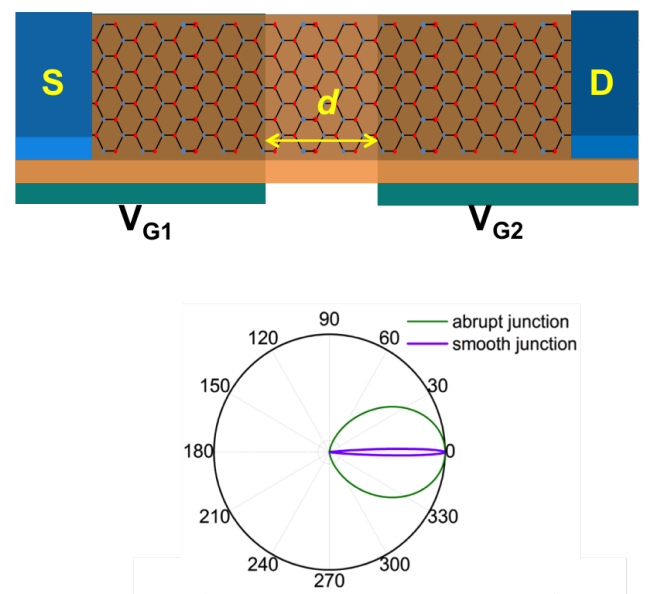

Figure 2.8: Impact of gate split on the angular transmission, electrons are collimated in a sense that only low angle electrons are transmitted.

perfect transmission demonstrating the difficulty in stopping the Klein tunnel mode (KTM), $\theta=0$, from going through. The prefactor in Eq. 2.7 becomes unimportant for moderate gate spacing $[44,45,42]$ and the total conductance can be calculated in the same procedure as Eq. 2.5

$$
\begin{aligned}
G & \approx \frac{4 q^{2}}{h} M\left(E_{F}\right)\left(\frac{1}{2}\right) \int_{-\theta_{0}}^{\theta_{0}} d \theta e^{-\pi k_{F} \frac{d}{2} \theta^{2}} \\
G & =G_{0}\left[\frac{1}{2 \sqrt{k_{F} d / 2}}\right] M
\end{aligned}
$$

where $M=k_{F} W / \pi$. Therefore the conductance is scaled by $\sim \sqrt{k_{F} d / 2}$ with a gate split (Fig. 2.9).

Figs. 2.10a,b show the doping-dependent resistance (from atomistic NEGF calculation) of a GPNJ for a 100 nm-wide graphene sheet, for abrupt and slowly varying potentials, respectively. In Fig. 2.10, we plot the variables against the shifts of the Dirac points in the two regions $\Delta E_{1}$ and $\Delta E_{2}$ from the Fermi Energy, $E_{F}$. The resistances at the upper left and lower right corners of the plot are higher than the other two ( $p n$ vs uniformly doped), 

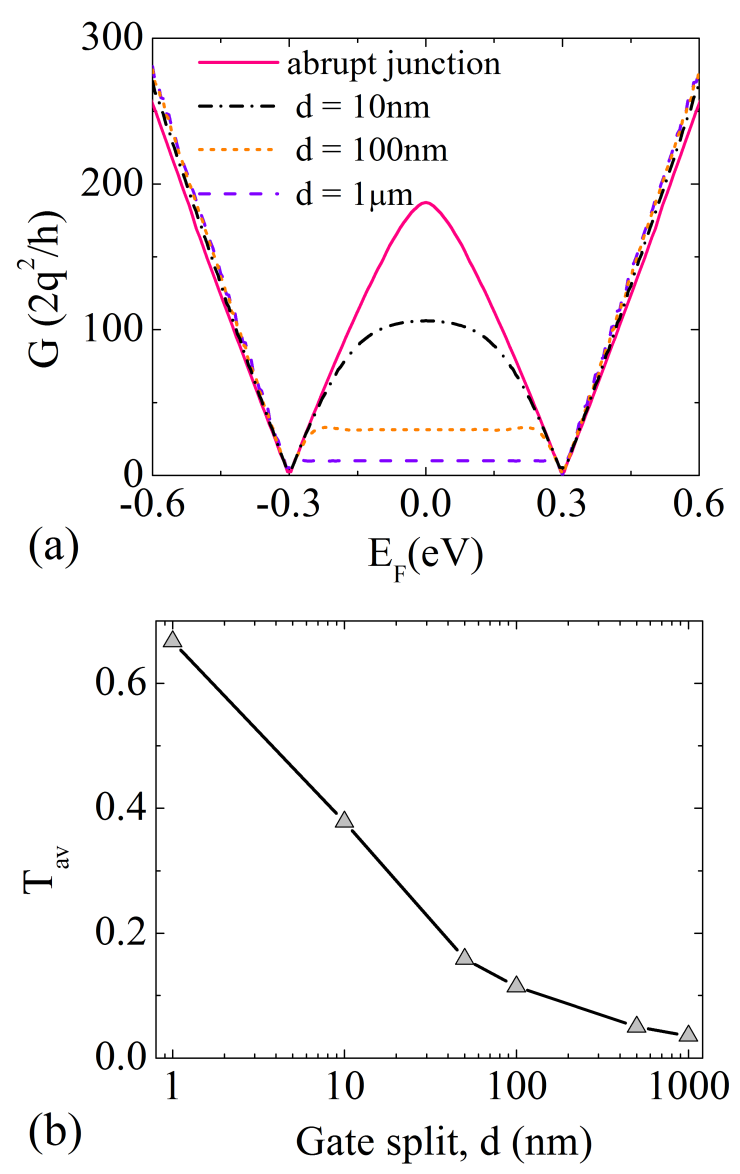

Figure 2.9: a) Conductance for various splits. b) How the conductance at the symmetric doping limit changes with gate split $\left(\sim \sqrt{k_{F} d / 2}\right)$

resulting an asymmetric resistance vs. doping in Fig. 2.10d (plotted for specific doping values $\Delta E_{1}$ as indicated with horizontal lines in 2.10b).

The WKB term in Eq. 2.7 is only present in the pn junction regime, and that is why only the $p n$ junction resistance is affected while going from abrupt to smooth junctions (Fig. 2.10a,b). For a $p^{+} p$ or $n^{+} n$ junction the Fermi energy does not cross the smoothly varying Dirac point anywhere in the device and the transmission expression only includes the wavefunction mismatch term. Fig. 2.10c shows the resistance variation for a fixed built-in potential $V_{0}=\Delta E_{1}-\Delta E_{2}$ (along black and red lines in Fig. 2.10a,b). In the conductance variation (inset), we see effectively two Dirac points, which has a simple physical explanation. 

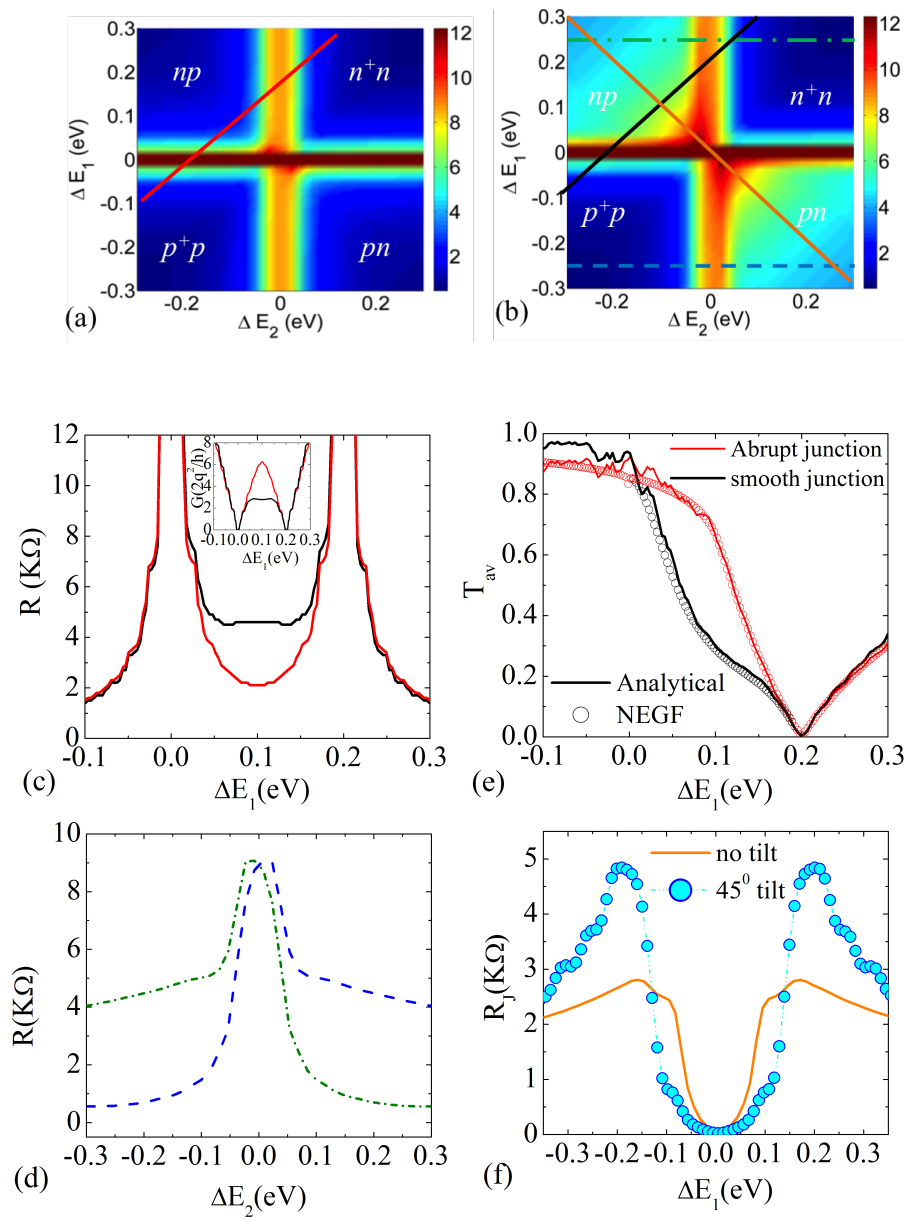

Figure 2.10: Numerical calculation (NEGF) of a single $p n$ junction conductance for both abrupt and smooth GPNJ. (a-b) Variation of total resistance with dopings in the two regions for abrupt (a) and smooth potential (c) Resistance as a function of Fermi energy $\left(E_{F}\right)$ for a fixed built in potential $V_{0}=\Delta E_{1}-\Delta E_{2}=0.2 \mathrm{eV}$. The red (abrupt) and black (smooth) lines are resistance plots along the corresponding black and red lines in (a) and (b). Conductance (inset) which can be viewed as $G=G_{0} M T_{a v}$ where $T_{a v}$, pinches off at two points - one due to vanishing $M$ and at another one due to vanishing $T_{a v}$, average transmission per mode. (d) The resistance asymmetry between $p n$ and $n n$ regime from plots along specific doping (specific $\Delta E_{1}$ lines from (b). (e) Extraction of $T_{a v}$ numerically. The transmission of a symmetric GPNJ is $2 / 3$ (at $\Delta E_{1}=0.1 \mathrm{eV}$ ), which results in asymmetric conductance vs doping in GPNJ. The solid lines are from NEGF and the circles are from analytical calculation, Eq. 2.11. (f) Junction resistance (Eq. 2.14) enhancement due to a tilt in the junction. This time we vary the doping along the diagonal (orange line in (b)) as done in the experiment [2].

Recall that the normalized conductance can be decoupled into the mode count from one end 
times the average transmission over to the other side

$$
G / G_{0}=M_{1} T_{12}=M_{2} T_{21}
$$

The left conductance minimum at $\Delta E_{1}=0$ is the point where $M_{1}$ becomes zero, while the right one at $\Delta E_{1}=0.2 \mathrm{eV}$ is the point where the average transmission for all modes, $T_{12}$, becomes zero. To make this clear, we calculate $T_{a v}$ numerically. Fig. 2.10e clearly shows a vanishing transmission at the second Dirac point. To calculate $T_{a v}$, we first simulate a graphene device with uniform doping and extract the overall mode count from the ballistic conductance $\left(G_{1}=M G_{0}\right)$. We then simulate the device with different dopings (finite builtin potential, $V_{0}$, and conductance $\left.G_{2}=G_{0} M T_{a v}\right)$. The ratio of $G_{2}\left(E_{F}\right)$ and $G_{1}\left(E_{F}\right)$ at each energy $E_{F}$ yields $T_{a v}\left(E_{F}\right)$. As we have discussed earlier, a symmetric $p n$ junction has $T_{a v}=2 / 3$, which is also reproduced by numerical calculation (Fig. 2.10e). In Fig. 2.10e we show analytical and numerical calculation on the same plot. They agree quite well.

\subsubsection{Conductance of a tilted $p n$ junction: high pass filter}

In this section we focus on a tilted GPNJ to show that it works as a high pass filter by allowing higher angle modes with higher transmission. We show that the junction resistance (similar to the odd resistance shown in other experiments $[45,42]$ ) is higher than the non-tilted device (Fig. 2.11). We argue that this enhancement originates from the chiral nature of graphene electrons which manifests itself through the highly angle dependent transmission characteristics of GPNJ (Fig. 2.12). The angular transmission lobe, oriented perpendicular to the interface is rotated with the tilt, where fewer transmitting modes exist. Therefore the conductance modulation would not occur for non-chiral, non-relativistic electrons with isotropic transmission. The results follow closely with recent transport measurements of a tilted GPNJ in a structure that has separately controlled split-gate voltages [46, 2]. We present an exact analytical solution to the spinor mismatch problem as well as NEGF based 
(a)

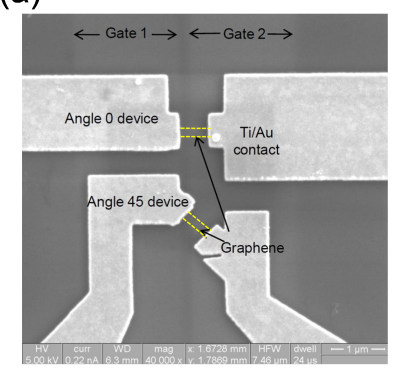

(c)

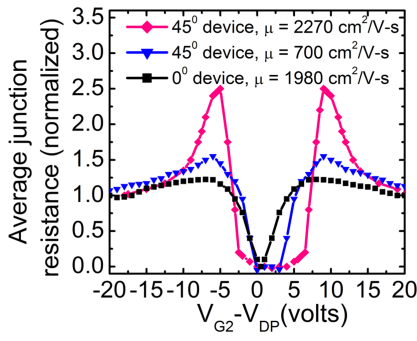

(b)

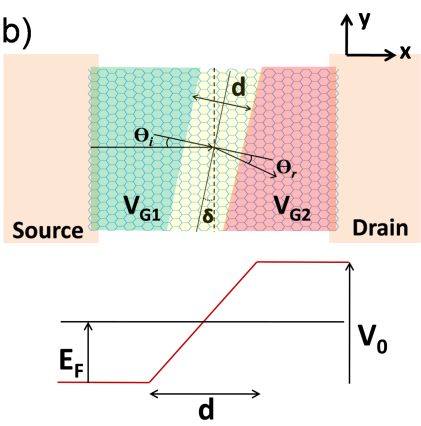

(d)

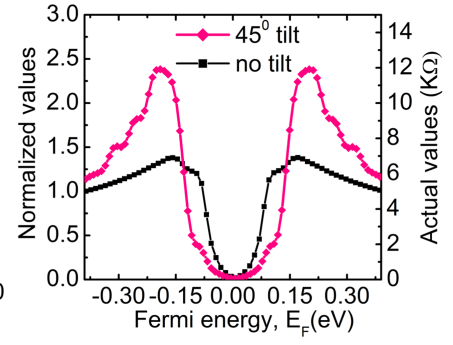

Figure 2.11: (a) Experimental setup in Ref. [2] for two devices, no tilt (upper), $45^{0}$ tilt (lower). The gate oxides are buried (runs vertically in the picture), (b) experimental and (c) theoretical calculation of junction resistance as a function of gate voltage. Resistance is higher for the tilted device compared to non tilted device, (d) Comparison of theoretical (solid lines, Eq. 2.13) and experimental (dots) average transmission per mode $T_{a v}$.

atomistic calculation for device sizes quite close to the experimental dimensions. We also find that charged impurity scattering dilutes, but does not eliminate the modulation in conductance (Figs. 2.11, 2.13). (iii) Notably, we demonstrate that multiple scattering events at edges can reverse the trend in modulation, giving an interfacial resistance that decreases with tilt (Fig. 2.14). Such decrease has been seen [47], but its physical origin has not been identified so far. The absence of such a reversal in experiments surprisingly points to its elimination, possibly through incoherent scattering processes dominant at the strained edges. Analytical model. The conductance of a GPNJ can be written as, $G=G_{0} \sum_{i}^{M} T\left(\theta_{i}\right)$ where $G_{0}=4 q^{2} / h$ is the conductance quantum including spin and valley degeneracies and $T\left(\theta_{i}\right)$ is the incident angle dependent transmission probability with $\theta_{i}=\tan ^{-1}\left(k_{y} / k_{x}\right)$. $M$ is the number of modes from the incident side for a given Fermi energy $E_{F}$ relative to its Dirac point and can be approximated as $M \approx W\left|E_{F}\right| / \pi \hbar v_{F}$ over the linear E-k regime. To recap, the angle dependent transmission $T$ is obtained by pseudospin conservation across the junction 

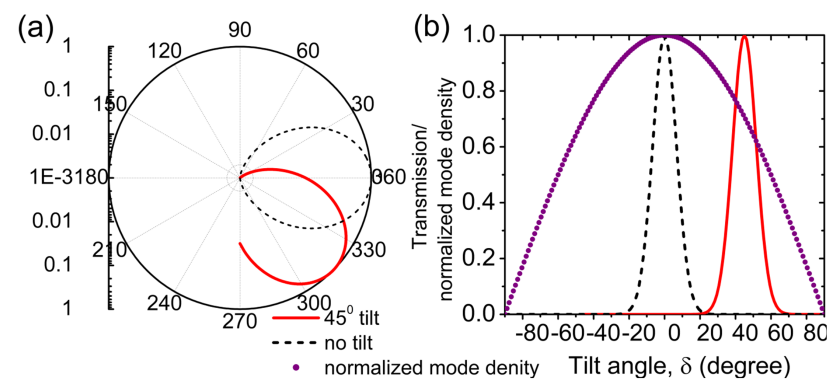

(c)

(d)
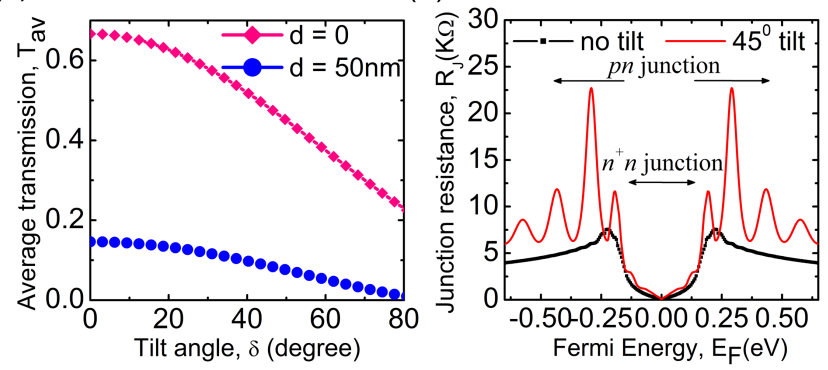

Figure 2.12: (a) Angular transmission for various tilt angles. (b) With tilt, the transmission lobe moves into a low mode density $(\sim \cos \theta)$ area giving $(\mathrm{c})$ a gradual decrease in transmission (Eq. 2.13) for a symmetric pn junction, (d) junction resistance at 0K predicted from Eq. 2.14. We see resonances which become more pronounced as we go into smaller systems.

$[40]$

$$
T\left(E_{F}, \theta_{1}\right)=\left[\frac{\cos \theta_{1} \cos \theta_{2}}{\cos ^{2}\left(\frac{\theta_{1}+\theta_{2}}{2}\right)}\right] e^{-\pi \hbar v_{F} k_{F}^{2} d \sin ^{2} \theta_{1} / V_{0}}
$$

This is a general form of the transmission expression in $[38,43]$ and works for the entire voltage range from $p n$ to $n n$ junction. The incident and refracted angles $\theta_{1,2}$ are related by Snell's law [34], $E_{F} \sin \theta_{1}=\left(E_{F}-V_{0}\right) \sin \theta_{2}$ (Eq. 2.1), $V_{0}$ is the voltage barrier across the junction and total wavevector $k_{F}=E_{F} / \hbar v_{F}$. For the rest of the dissertation, we use the average transmission of all modes defined as, $T_{a v}=G / M$. For a tilted junction, the incoming mode angles are modified, so that the conductance becomes

$$
G\left(E_{F}\right)=G_{0} \sum_{i}^{M\left(E_{F}\right)} T\left(E_{F}, \theta_{i}+\delta\right)
$$


where $\delta$ is the tilt angle as shown in Fig. 2.11. In Eq. 2.12 the effective split between the two gates is $d / \cos \delta$. As a result of the angle dependence, the transmission lobe at a particular energy will rotate by the tilt angle (Fig. 2.12a). The transverse wavevector $k_{y}=k_{F} \sin \theta$ gives $\Delta \theta=\Delta k_{y} / k_{F} \cos \theta$, so that the mode density decreases as we go to higher angles relative to the transport axis. A tilt at the junction thus shifts the transmission window onto a high angle region where the mode density is less, decreasing the overall transmission (Fig. 2.12c). In the limit when the number of modes is very few, the experiment will give the mode resolved angle dependent transmission properties (Fig. 2.12a). For an abrupt, symmetric pn junction, the transmission expression reduces to $\cos ^{2} \theta$ from Eq. 2.11 and it is easy to see the impact of tilt,

$$
G \approx G_{0} \int_{-\pi / 2}^{\pi / 2-\delta} \frac{T(\theta+\delta)}{\Delta \theta} d \theta=G_{0} \frac{2}{3} \cos ^{4}\left(\frac{\delta}{2}\right) M
$$

The factor $2 / 3$ arises from the wavefunction mismatch across the junction and the tilt introduces an additional scaling factor, which further reduces conductance. The gradual decrease of $T_{a v}$ with $\delta$ in Fig. 2.12c constitutes a direct manifestation of chiral tunneling in graphene.

To connect with experimental measurements [2], we next analyze the variation of the junction resistance in the presence of an intrinsic background doping $\left(V_{D P}\right)$ in the graphene sheet (Fig. 2.12d). We vary the gate voltages so that $V_{G 1}=-V_{G 2}$ but a nonzero $V_{D P}$ makes it an asymmetric GPNJ. The effective gate voltages on the graphene sheet are $\alpha_{G}\left(V_{G 1}+V_{D P}\right)$ and $\alpha_{G}\left(V_{G 2}+V_{D P}\right)$, where $\alpha_{G}$ is the capacitive gate transfer factor. The junction resistance can be written as [48]

$$
R_{J}=\left(\frac{4 q^{2}}{h}\right)^{-1}\left[\frac{1-T_{a v}}{M T_{a v}}\right]
$$

Fig. 2.12d plots $R_{J}$ against $E_{F}=\hbar v_{F} \sqrt{(\pi) \alpha_{G} C_{G}\left|V_{G 1}\right| / q}$, the amount of shift in the Dirac point by $V_{G 1}$, for a $100 \mathrm{~nm}$ wide graphene sheet with a split gate separation $d=200 \mathrm{~nm}$. The 
voltage range $\left|V_{G 1}\right|<V_{D P}$ is in the $n^{+} n$ regime for positive $V_{D P}$ and $p^{+} p$ for negative. Under these near homogeneous conditions, the junction resistance predicted by Eqs. 2.11-2.14 is small, because the pseudospin states match and there is no WKB tunneling term in Eq. 2.11. $\left|V_{G 1}\right|>V_{D P}$ is in the $p n$ junction regime and resistance jumps to a high value, primarily due to the WKB factor in Eq. 2.11 (similar trend was seen in $[45,42]$ ). The rate of change in $R_{J}$ with $V_{G 1}$ is determined by gate split. For the $45^{0}$ tilted junction, the junction resistance is higher than the non-tilted resistance. We see oscillation in the resistance (Fig. 2.12d) for the single $n p$ junction. This is different from the interference oscillation in Ref. [41] for the resonant cavity formed in an npn strucure. This can be understood from the conductance in the $p n$ junction regime, simplified as $G\left(k_{F}\right) \approx G_{0} \sum_{i}^{M} \exp \left(-\pi k_{F} d \sin ^{2} \theta_{i} / 2\right)$ for large gate split d. With increasing gate voltage (higher $k_{F}$ ) there are more modes $(M)$ in the summation with each mode transmitting with exponentially reduced magnitude. The two opposing effects generate a sequence of peaks and valleys and dominate when summed over a few modes (either with quantization or tilt) at low temperature.

Numerical model. An atomistic, numerical calculation of the junction resistance is shown in Fig. 2.11 at $80 \mathrm{~K}$ temperature. NEGF formalism is used for a $100 \mathrm{~nm}$ wide graphene sheet with $d=200 \mathrm{~nm}$, close to experimental dimensions (width $\sim 200-300 \mathrm{~nm}$ ). A single $p_{z}$ orbital basis for each carbon atom is used to compute the Hamiltonian $H$, while the contact self-energies $\Sigma_{1,2}$ are calculated using a recursive technique. The retarded Green's functions are calculated as $G^{R}=\left(E_{F} I-H+V-\Sigma_{1}-\Sigma_{2}\right)^{-1}$ using the algorithm in [49] and $V$ is the electrostatic potential inside the device. In units of $2 q^{2} / h$, the conductance is calculated as $G=\Gamma_{1} G^{R} \Gamma_{2} G^{A}$, where the contact broadening functions, $\Gamma$ are the antiHermitian components of $\Sigma$. For ballistic transport, $G$ equals $M$, the number of modes for a uniformly gated sheet, and $M T_{a v}$ for a $p n$ junction. Combining these two, we calculate $T_{a v}$ and $R_{J}$ from Eq. 2.14, which shows a jump with tilt in the pn junction regime, very similar to the experiment.

Charged impurity scattering dilutes tilt dependence. The experimental device is on a $\mathrm{SiO}_{2}$ 

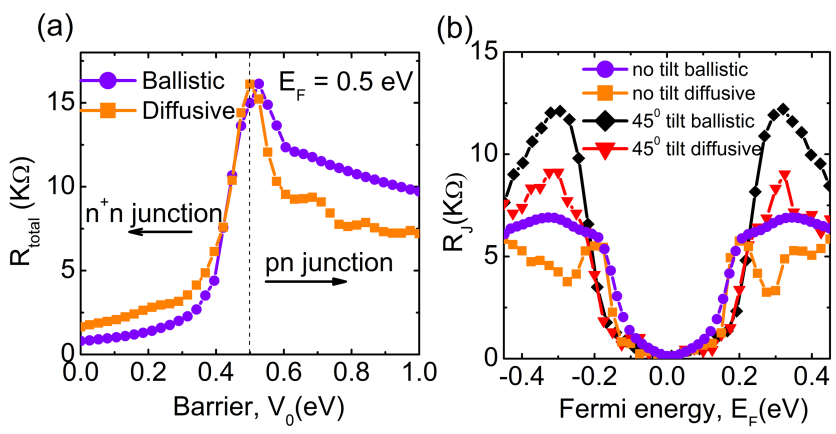

Figure 2.13: Impact of charged impurity scattering, (a) conductance asymmetry is diluted due to impurity potentials, the ballistic resistance is normalized for comparison. (b) Reduced asymmetry results in lower junction resistance for both tilted, non-tilted devices, thus retaining their difference.

substrate and the transport is diffusive with a mobility varying from $700-3000 \mathrm{~cm}^{2} / \mathrm{V}$-s. It is natural to inquire how the theoretical model, which so far does not include scattering, corresponds to experiments. To explore this feature, we included the impact of charged impurity scattering in our model. We use a sequence of screened Gaussian potential profiles for the impurity scattering centers [50, 51, 52], $U(r)=\sum_{n=1}^{N_{i m p}} U_{n} \exp \left(-\left|r-r_{n}\right|^{2} / 2 \zeta^{2}\right)$, that specifies the strength of the impurity potential at atomic site $\mathrm{r}$, with $r_{n}$ being the positions of the impurity atoms and $\zeta$ the screening length $(\approx 8$ times the $\mathrm{C}$-C bond for long range scatterers). The amplitudes $U_{n}$ lie in the range $[-\delta, \delta](\approx 0.5$ times the $\mathrm{C}$-C coupling parameter) and $N_{i m p}$ is the impurity concentration $\left(\sim 5 \times 10^{11} \mathrm{~cm}^{-2}\right)$. Note that the purely diffusive model discussed in $[53,42,45]$ ignores the quantum mechanical spinor mismatch and WKB scaling and therefore underestimates the junction resistance for cleaner samples. Our NEGF based numerical model on the other hand captures both the quantum mechanical and impurity limited resistance contributions simultaneously. The junction resistance is now calculated by eliminating the contact and device resistance [2]

$$
\begin{gathered}
R_{J}=\left[R\left(V_{G 1}, V_{G 2}\right)+R\left(V_{G 2}, V_{G 1}\right)\right. \\
-R\left(V_{G 1}, V_{G 1}\right)-R\left(V_{G 2}, V_{G 2}\right] / 2,
\end{gathered}
$$


where the first two terms contain the junction resistance, while the last two do not.

Fig. 2.13a shows the impact of the impurity scatterings on the total resistance and Fig. 2.13b on the junction resistance. We take the average resistance over many impurity configurations. This puts a constraint on the computation size, so we show calculations for a smaller device (50 nm wide). We find that both tilted and non-tilted junction resistances are suppressed, thereby retaining the difference between the two. This reduction in junction resistance with scattering is quite consistent with the experiment (Fig. 2.11c, red line is for a $45^{0}$ device with mobility $2270 \mathrm{~cm}^{2} / \mathrm{V}$-s, while the blue triangle is for $45^{0}$ with lower mobility, $\left.700 \mathrm{~cm}^{2} / \mathrm{V}-\mathrm{s}\right)$.

The reduction in the junction resistance from ballistic to diffusive transport can be understood from the total resistance, shown in Fig. 2.13a. Now we keep $V_{G 1}$ fixed and vary $V_{G 2}$ so that we go from $n^{+} n$ to $n p$ junction. We see a clear asymmetry in the $\mathrm{R}-\mathrm{V}_{G}$ characteristics $[38,42]$ (purple line normalized to the orange line in Fig. 2.13a for comparison). The asymmetry confirms the presence of $p n$ junction, which reduces the conductance due to spinor mismatch. The presence of impurity scattering reduces this asymmetry while increasing the overall resistance (red line). The impurity potentials create a random potential variation throughout the graphene sheet on top of the applied gate voltages, thus blurring the presence of a pn junction. Therefore the resistance due to spinor mismatch becomes less noticeable (Fig. 2.13b). Indeed, the experimental data of the total resistance indicates an increase in asymmetry in the tilted junction [2], signifying an increase in the junction resistance.

Edge scattering can reverse tilt dependence. A striking feature on the experimental results is their agreement with Eq. 2.13. This match is remarkable, considering that the equation was derived assuming no edge reflections and the fact that past numerical study [47] showed in fact an increase in conductance with tilt. We argue that the above reversal of junction conductance with tilt is entirely due to edge scattering events. Indeed, from an atomistic NEGF calculation with shorter widths than lengths, we find that the transmission shows a 
(a)

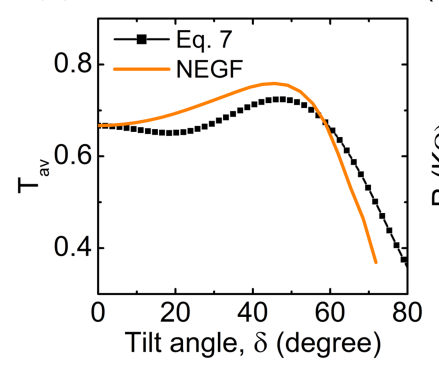

(b)

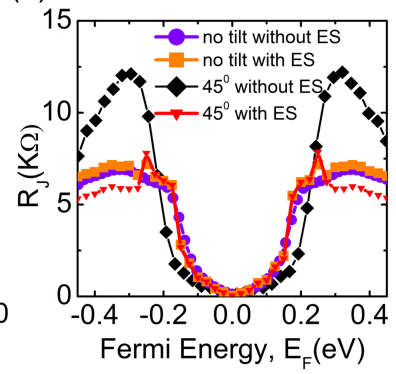

(c)

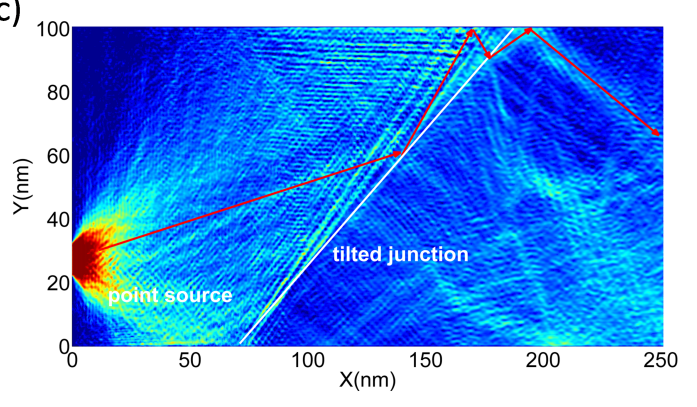

Figure 2.14: (a) Increase in conductance in a tilted GPNJ due to Edge Scattering (ES) in contrast with Fig. 2.12c, (b) corresponding decrease in junction resistance due to tilt (c) Mechanism of edge enhanced conductance for a tilted junction from atomistic NEGF calculation: some reflected electrons come back at the junction after edge reflection.

pronounced local maximum (Fig. 2.14a orange line) and increase in junction resistance, in agreement with [47]. We summarize this in Fig. 2.14b, where an increasing tilt makes the resistance increase for the short channel $125 \mathrm{~nm}$ x $50 \mathrm{~nm}$ device (a transition from purple circle to black diamond), but decrease for the longer $200 \mathrm{~nm}$ x $50 \mathrm{~nm}$ device (orange square to red triangle). Bearing in mind that the gate split is $100 \mathrm{~nm}$, the short channel device significantly reduces edge scattering.

To better understand the origin of such a resistance reversal, we inject electrons with a small contact at the left edge (bright red spot in Fig. 2.14c) and plot the spatial current density under a small drain bias. The numerically computed electron trajectories show how a tilt can enhance forward scattering events at the edge and thus an increase in conductance. The enhancement arises from simple 'geometrical optics' dictated by Snell's law. We can identify the incident wide angle modes $(\theta>\pi / 4-\delta)$, for which the reflected 'ray' hits the upper edge with a positive $x$ directed velocity. Such a mode will reflect back toward the 
junction again. The contribution from the positively directed edge scattering event is given by

$$
G_{\text {edge }}=G_{0} \int_{\pi / 2-3 \delta}^{\pi / 2-\delta} \frac{T(\pi-3 \delta-\theta)}{\Delta \theta} d \theta=2 \sin ^{4} \delta \cos \delta M
$$

Note that only the incident angles below the critical angle are considered while setting the limits of the integration. With the added contribution from edge scattering, the net mode-averaged transmission $T=G / M$ is given by

$$
T_{\text {total }} \approx T+(1-T) T_{\text {edge }} \eta
$$

where the $T$ s are extracted from the corresponding $G / M$ ratios and $\eta$ is a parameter that describes the efficiency of edge scattering. In the absence of edge scattering $(\eta=0), T_{\text {total }}=T$ and decreases with tilt (Fig. 2.12c). However in the presence of strong edge scattering $(\eta=1)$, the added forward edge scattering term in Eq. 2.17 closely reproduces the NEGF result with the local transmission maximum (Fig. 2.14a, black dotted line). Comparing these results with experiment indicates that such edge scattering events are clearly minor. We conjecture that the coherent forward scattering processes captured by NEGF can be diluted in the experiments by the presence of incoherent scattering processes arising at the strained and rough edges of the graphene samples that tend to dephase or perhaps even trap the electrons.

In the following chapter, we discuss how we combine the two filters to form a transmission gap and a high current modulation in graphene without opening a bandgap.

\section{Related publications:}

1. Redwan N. Sajjad, S. Sutar, J. Lee and Avik Ghosh, "Manifestation of Chiral tunneling at a tilted graphene p-n junction", Physical Review B vol. 86, pp. 155412(2012). 


\section{Chapter 3}

\section{Transmission gap: a new way of switching}

In this chapter, we propose device concepts that produce a transmission gap to suppress all propagating modes. We show two device designs that achieve this goal.

The conductance at zero temperature can be written as,

$$
G\left(E_{F}\right)=G_{0} \sum_{n=1}^{M\left(E_{F}\right)} T_{n}=G_{0} M T_{a v}
$$

where $G_{0}=4 q^{2} / h$ is the conductance quantum which includes spin and valley degeneracy, $M$ is the total number of transverse modes and $T_{n}$ is transmission of individual modes. If all modes transmit with equal probability $(T)$, the conductance can simply be written as $G_{0} M T$. As we discussed earlier, due to the chiral nature of carriers in graphene, transmission in a graphene $p n$ junction (GPNJ) is highly angle (mode) dependent, making it necessary to work with an average transmission per mode $T_{a v}$ over all modes. Instead of eliminating the mode count $M$ as does a structural band-gap, we exploit instead the chiral (anisotropic) tunneling that makes $T_{a v}$ vanishingly small (Fig. 3.1b) over a range of energies. This results in low OFF current (Fig. 3.1c,d). All modes are available for conduction in the ON state when the 
split gates are set to the same polarity, thus retaining the high mobility of graphene.

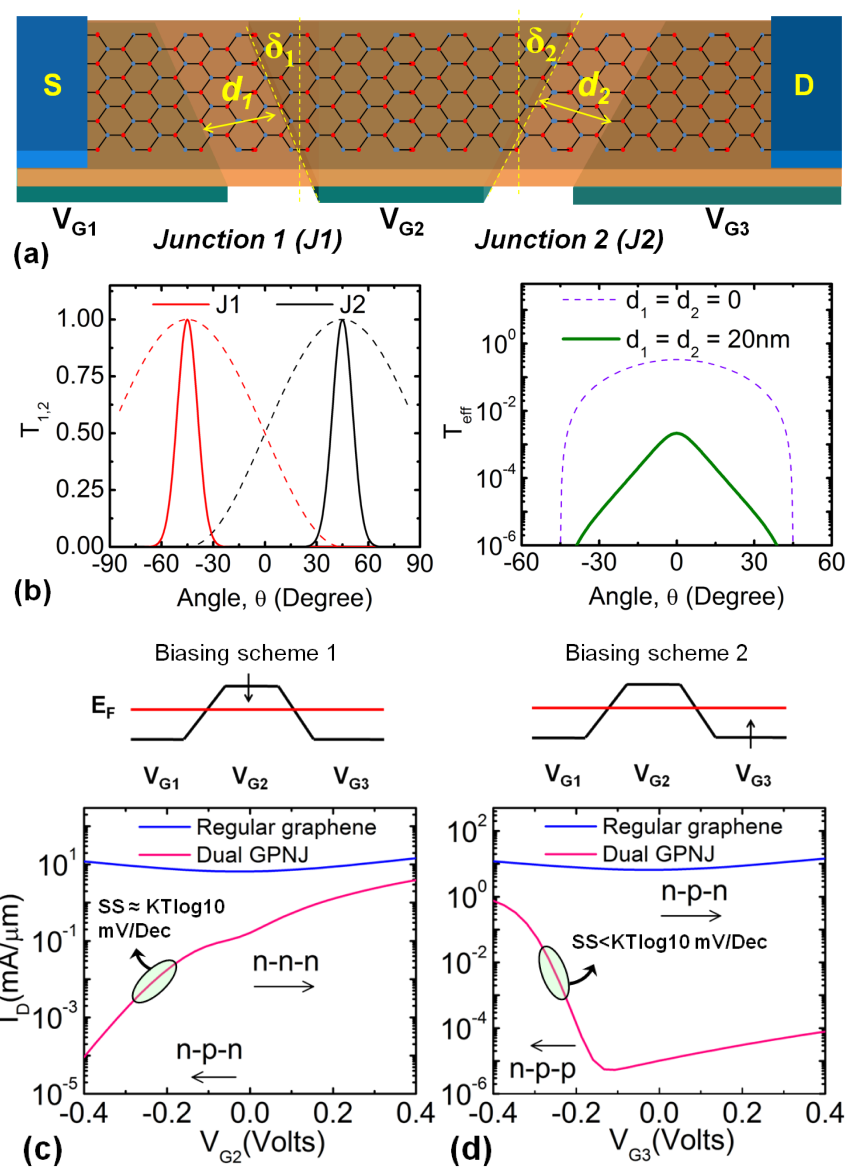

Figure 3.1: (a) Chiral tunneling in graphene pn junction (GPNJ) manipulated with gate geometry, using two junctions (dual GPNJ) tilted in opposite directions, (b) making their angle dependent transmission lobes orthogonal (left) and yielding negligible overall transmission (right) for split gates (solid line). (c) The transmission gap creates a high ON-OFF current ratio as a function of $V_{G 2}$ at finite bias, $V_{D S}=0.4 \mathrm{~V}$ and room temperature. The ON current degrades slightly compared to homogeneous gapless graphene, but the OFF current is reduced by several orders of magnitude (d) Steeper change (beating $K T \ln 10 / q$ switching limit) of current with $V_{G 3}$. This is done by keeping the collimation effect of the first junction intact and making the transmission gap dependent upon Snell's law.

\subsection{Opening transmission gap with gate geometry}

In this section, we combine a split gated $p n$ junction to collimate the transverse modes with the $[2,54]$ action of a tilted $p n$ junction that increases the effective angle of incident electrons. Fig. 3.1a shows two pn junctions tilted (at angle $\delta$ ) in opposite directions. Each junction 
exploits chiral tunneling that conserves the pseudospin index and maximizes transmission for normal incidence (Klein tunneling). Transmission is suppressed for non-zero incidence angles, especially when the potential varies smoothly, i.e., the $p$ to $n$ transition occurs over a finite split gate distance $d_{1}=d_{2}=2 d[43]$. A tilted junction rotates the transmission lobe accordingly [54], which shifts transmissions along opposite directions to make them orthogonal. The mode-averaged transmissions across the dual junction can be decomposed as (see Appendix B for derivation)

$$
\begin{aligned}
T_{1,2}(\theta) & \approx\left[\frac{\cos \left(\theta_{L} \pm \delta\right) \cos \theta_{R}}{\cos ^{2}\left(\frac{\theta_{L} \pm \delta+\theta_{R}}{2}\right)}\right] \\
& \times \exp \left[-\pi 2 d \frac{k_{F L} k_{F R}}{k_{F L}+k_{F R}} \sin \left(\theta_{L} \pm \delta\right) \sin \left(\theta_{R}\right)\right] \\
\frac{1}{T_{\text {eff }}} & \approx \frac{1}{T_{1}}+\frac{1}{T_{2}}-1 \\
T_{a v}\left(E_{F}\right) & =\frac{1}{2} \int T_{e f f}(\theta) \cos \theta d \theta \\
& =\left[A \sqrt{k_{F} d} e^{\pi k_{F} d \sin ^{2} \delta}\right]^{-1}
\end{aligned}
$$

$T_{a v}$ is vanishingly small for moderate doping. Eq. 3.4 is written for symmetric $n p n$ doping with Fermi wave-vector, $k_{F}=E_{F} / \hbar v_{F}$ and $A \approx 8$ is a constant. The angular representation of a mode is given by, $\theta=\tan ^{-1} \frac{k_{y}}{k_{x}}$, where $k_{y}$ and $k_{x}$ are the transverse and longitudinal components of $k_{F}$. Eq. 3.2 arises from matching the pseudospins across the junction. $L$ and $R$ denote components to left and right of a junction $(1,2)[55]$. The tilt $\delta$ modifies the incident angle by $\theta_{L} \pm \delta$ and the angle of refraction is related to incident angle through Snell's law, $k_{F L} \sin \left(\theta_{L} \pm \delta\right)=k_{F R} \sin \theta_{R}$. Eq. 3.3 assumes resistive addition of the junction resistances and ballistic flow in between. The mode count for width $W$ is given by $M=\frac{W k_{F}}{\pi}$. The resulting total conductance, $G_{0} M T_{a v}$, is negligible in the entire $p n$ junction regime, which indicates that the transmission gap $\left(E_{G}\right)$ exists if the carrier densities have opposite polarities,

$$
E_{G} \approx V_{0}
$$




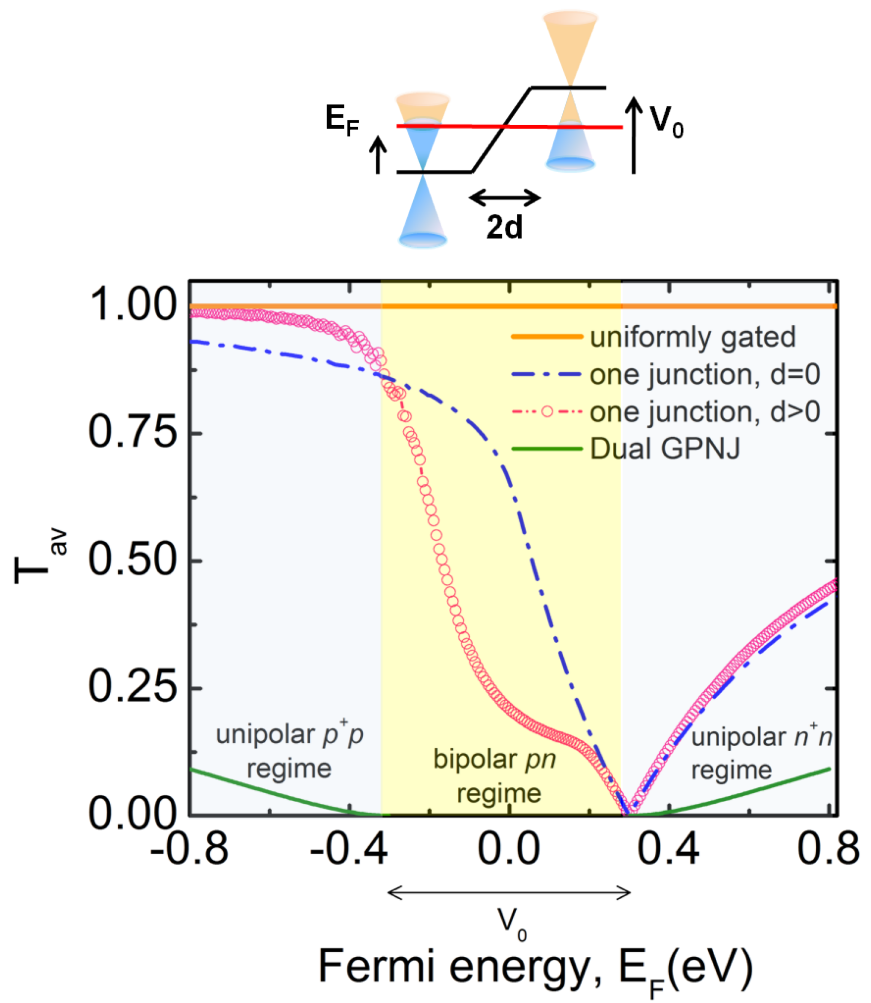

Figure 3.2: Mode-averaged transmission $T_{a v}$ vs Fermi energy $E_{F}$ for different doping profiles (Fermi energy $E_{F}$ and built in potential $V_{0}$ are indicated on the top band diagram). $T_{a v}$ for the dual tilt GPNJ shows a gap (green line), which is termed as transmission gap (yellow shading) in this chapter.

where $V_{0}$ is the gate induced potential step across the junction. This is due to the fact that the high resistance is primarily contributed by the WKB exponential factor which is present in the $p n$ regime, whereas the unipolar regime has only the cosine prefactors representing the wavefunction mismatch $[38,55]$. Significantly, Eq. 3.2 predicts two critical angles for total internal reflection: a Snell's law driven $\theta_{C}=\sin ^{-1}\left(n_{R} / n_{L}\right)$ arising from the prefactor and a second smaller $\theta_{C} \sim 1 / \sqrt{\pi k_{F} d}$ from the WKB term.

Fig. 3.2 shows variation of $T_{a v}$ numerically calculated from Eq. 3.2 as a function of Fermi energy, $E_{F}$ for four different devices and doping profiles. The orange line shows unit transmission of all modes for a ballistic uniformly doped graphene sheet. The angular (mode dependent) transmission is manifested in a single sharp $(d=0)$ graphene $p n$ junction and the $T_{a v}$ is suppressed (blue dash). Further suppression is achieved with a split junction (pink 
circles) (non-zero $d$ ) due to high transverse energy (mode) filtering. $T_{a v}$ for the device in Fig. 3.1a is shown in green, showing a negligible transmission over the bipolar doping regime. Note that the suppression in transmission only occurs in the bipolar doping regime (Fig. 3.2), outside which the exponential scaling in Eq. 3.2 is eliminated [55].

The minimum current is achieved in npn regime (OFF state). Over the energy window $\left[\mu_{S}, \mu_{D}\right]=\left[E_{F}, E_{F}-q V_{D S}\right]$ set by the drain voltage $V_{D S}, T_{a v}$ varies weakly, so that the OFF state current at zero temperature for the npn configuration can be extracted from

$$
\begin{aligned}
I_{O F F}= & G_{0} \int_{\mu_{D}}^{\mu_{S}} M(E) T_{a v}(E) d E \\
& \approx G_{0} M\left(E_{F}\right) T_{a v}\left(E_{F}\right) V_{D S}
\end{aligned}
$$

convolved with the thermal broadening function for finite temperature. For uniformly doped graphene with ballistic transport,

$$
I_{O N}=G_{0} M\left(E_{F}\right) V_{D S}
$$

so that the zero temperature ON-OFF ratio simply becomes,

$$
I_{O N} / I_{O F F} \approx\left[T_{a v}\left(E_{F}\right)\right]^{-1} \sim A \sqrt{k_{F} d}\left(2 e^{\pi k_{F} d \sin ^{2} \delta}\right)
$$

if the biasing is changed all the way from npn to $n n n$. Fig. 3.1c (Pink) shows the change in dual tilt GPNJ current with gate voltage $V_{G 2}$ at room temperature and finite drain bias $\left(V_{D S}\right)$, compared with a regular zero bandgap graphene based device (Blue). From the nin to $n n n$ regime, we see little change in GPNJ current on a log scale. But towards the npn regime, we see at least three orders of magnitude decrease when the Fermi window remains mostly within the transmission gap. Compared to regular graphene (Blue), the ON current is reduced only slightly, while the OFF current is reduced by several orders of magnitude. The reduction in $\mathrm{ON}$ current is due to the fact that the doping is not quite uniform at the $\mathrm{ON}$ 


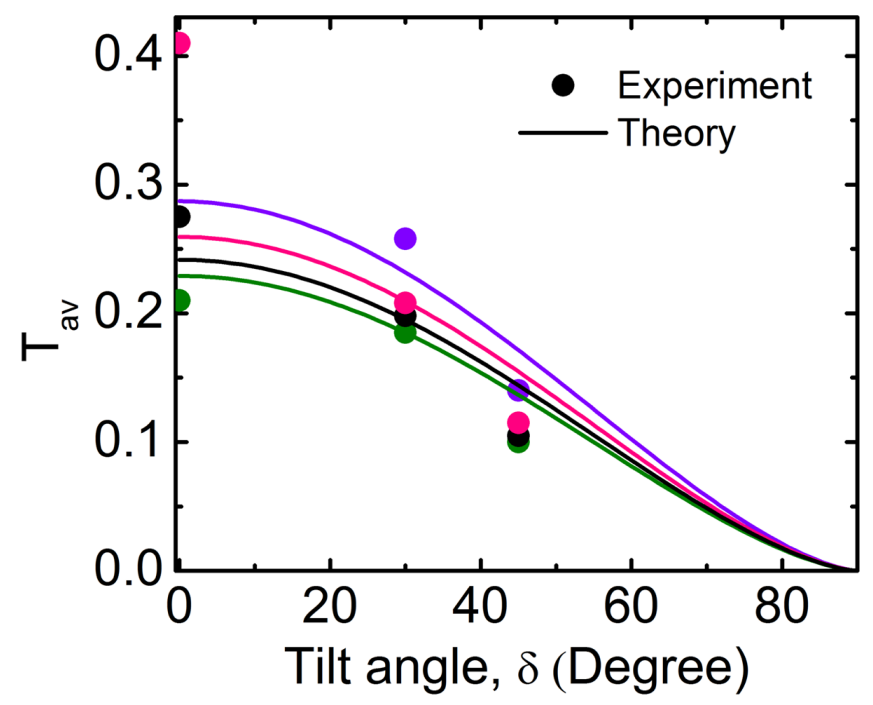

Figure 3.3: Benchmarking $T_{a v}$ with experiment [2] for a single tilted split junction for several gate voltages. Experiment shows good agreement with the theory confirming the angular shift of $T(\theta)$ and the scaling law with tilt.

state across the $n^{+} n$ collimator which is maintained at unequal doping to avoid a large voltage swing, whereupon the wavefunction mismatch leads to lower current than uniform graphene. Fully ballistic transport and assuming high quality contact yields an intrinsic ON current in the $\mathrm{mA} / \mu \mathrm{m}$ regime. In this calculation the gate parameters are $\left|\delta_{1}\right|=\left|\delta_{2}\right|=\delta=45^{0}$, $d_{1}=d_{2}=20 \mathrm{~nm}, V_{G 1}=V_{G 3}=+1 \mathrm{~V}, V_{D S}=0.4 \mathrm{~V}$.

Critical to the geometric switching is the prominence of angle-dependent chiral transmission across a tilted junction. Fig. 3.3 shows the mode averaged transmission $T_{a v}$ extracted (Appendix B) from the measured junction resistance for a single split junction, for varying tilt angles [2]. For an abrupt tilted junction $T_{a v}=\frac{2}{3} \cos ^{4}(\delta / 2)$ in the symmetric $p n$ doping limit and represents an electronic analog of Malus' law, the quenching of light transmission through an polarizer-analyzer pair. The reduction in $T_{a v}$ originates from the angular shift of the transmission lobe (Fig. 3.1b) in the low angular mode density region [54]. The numerically evaluated $T_{a v}$ generalized for a tilted split junction (solid lines) agrees well with experimental data (dots) for different gate voltages. The scaling of $T_{a v}$ in the experiment thus confirms the angular shift of the transmission lobe and forms the basis of the proposed device. The data shows an absence of specular edge scattering and persistence in the presence of charge 
puddles. The detailed theoretical model can be found in Ref. [54].

\subsection{Biasing scheme and impact on subthreshold slope}

For a semiconductor with fixed bandgap, rate of change of current with gate voltage is $K T \ln 10 / q$ and limits the energy dissipation in binary switching. The limit arises from the rate of change in overlap between the band-edge and the Fermi-Dirac distribution, normally set by the Boltzmann tail. In our device, the transmission gap is sensitive to how gate voltages are varied, since it is a gate-voltage-dependent transport gap. There can be two ways to turn ON the device by getting rid of both junctions (Fig. 3.1c, biasing scheme 1: vary $V_{G 2}$ for $n p n \rightarrow n n n$ ) or only one junction (Fig. 3.1d, biasing scheme 2 : vary $V_{G 3}$ for $n p n \rightarrow n p p$ ). Earlier we showed the transfer curve $I_{D}-V_{G}$ (Fig. 3.1c) as a function of $V_{G 2}$. Changing $V_{G 2}$ compromises the collimation action of the first junction and the condition of transmission gap is decided by the bipolarity (Eq. 3.5). Therefore the effective conduction band moves by the same amount as the applied gate voltage, yielding $\sim K T \ln 10 / q \mathrm{mV} /$ Dec change in current. In Fig. 3.1d, $V_{G 3}$ is changed and a drastically different transfer characteristic emerges. For simplicity, we keep the first junction split gated but without any tilt. The first junction limits transmission primarily around normal incidence $(\theta \approx 0)$, while the second junction, tilted at $\delta$, increases the effective angle of incidence by the gate tilt angle $\delta$ [54]). All the electrons are then reflected if the critical angle of the second junction is less than $\delta$,

$$
\theta_{C}=\sin ^{-1}\left|\frac{n_{R}}{n_{L}}\right|<\delta
$$

where $n_{R}$ and $n_{L}$ are doping concentrations on the two sides of junction 2 . The resulting transmission vanishes over a range of energies (following from Eq. 3.9), which can be expressed as $[40]$

$$
E_{G}=V_{0} \frac{2 \sin \delta}{\cos ^{2} \delta}
$$




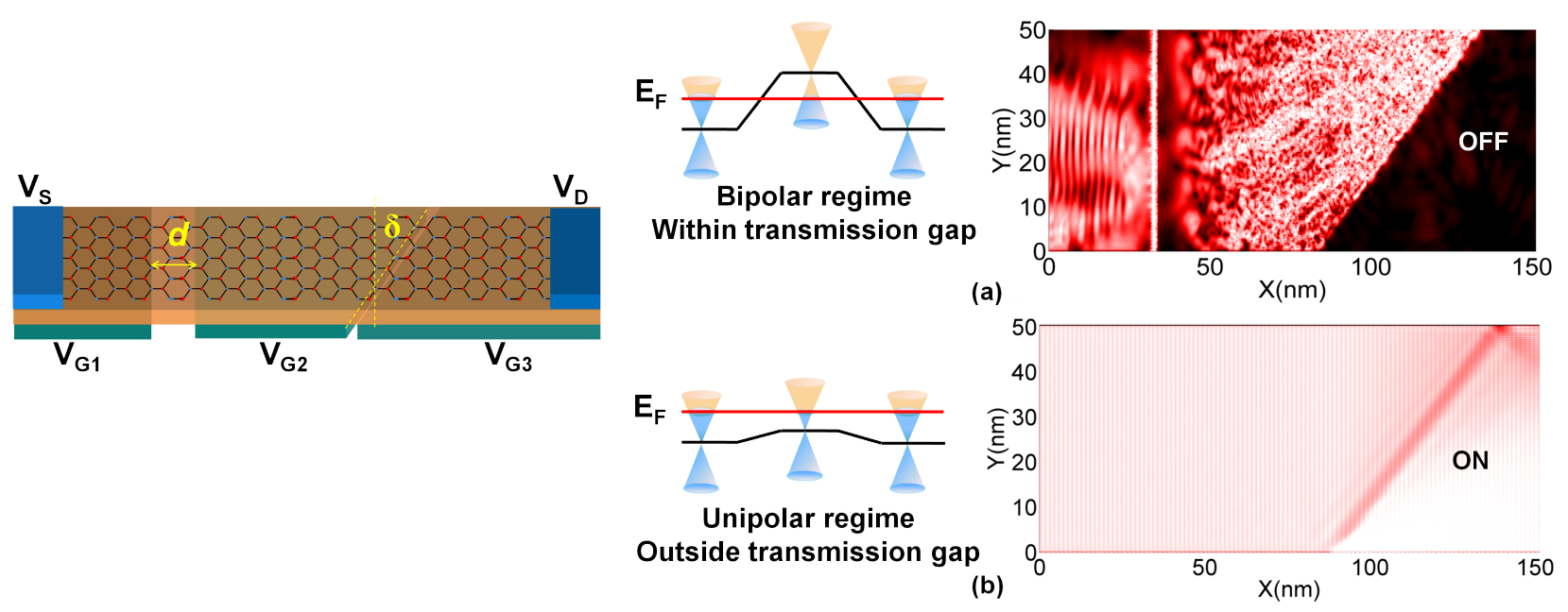

Figure 3.4: Electron flow in the proposed GPNJ device. Schematic of collimator-barrier pair that sequentially filters all propagating modes is shown on left. Numerical current density plot from NEGF showing (a) blocking of carrier flow in the bipolar npn OFF state, (b) unipolar $n n^{-} n$ ON state, current flowing from source to drain. White (black) areas indicate high (low) local current density.

analogous to Ref. [40] despite being a different (simpler) geometry, with the tilt angle $\delta$ replacing the barrier angle $\theta_{B}$. Such tunability of the transmission gap for an abrupt junction bears a direct impact on the sub-threshold slope. Once we enter the unipolar regime for the second junction ( $p n$ to $p p$ ), the critical angle from Snell's law (discussed earlier) comes into play and the effective band edge shifts by $-q \cdot V_{G 3} /(1-\sin \delta)$ at the thin oxide limit. That means the transmission gap overlaps with the Fermi distribution at a higher rate than usual with change in gate bias, leading to a subthermal slope, $\mathrm{SS}=\alpha K T \ln 10(1-\sin \delta) / q[40]$ steeper than the Landauer limit, where $\alpha$ is inversely proportional to the filtering strength $\left(\sqrt{k_{F} d}\right)$ of the first junction. However, since the first junction is always present under this scheme, it will reduce the ON current and the ON-OFF ratio by a factor of $\sqrt{k_{F} d}[43]$ compared to Eq. 3.7-3.8.

To demonstrate carrier trajectories in the proposed device, we numerically simulate the device (150 nm x $50 \mathrm{~nm}$ ) using the Non-Equilibrium Green's function Formalism (NEGF). Fig. 3.4a,b shows the local current density. The source and drain Fermi levels are at $\mu_{S}=0$ and $\mu_{D}=-q V_{D S}$. To visualize the current distribution in the device at the Fermi level, 
we apply a small drain bias $V_{D S}$ so that all electrons are at the same quasi-Fermi level (as indicated by the red line in Fig. 3.4). The $n n^{-} n$ ON state (Fig. 3.4b) shows current from source to drain while the npn OFF state (Fig. 3.4a) shows very little current inside the final wedge connected to the drain. Most of the electrons that do not cross the tilted junction are redirected toward the source. Some electrons, especially the secondary modes $(\theta>0)$, are rejected by the initial collimator after edge reflections and tend to build up in the central wedge. The build-up of carriers increases the local quasi-Fermi level, $\mu$, until the injection rate at the left junction, which is set by the transmission rate in Eq. 3.2, equals the leakage rate at the right tilted junction. The leakage is given by the exponentially reduced transmission in Eq. 3.4 plus additional edge scattering based pathways (a model was presented in Ref. [54] including an edge reflection parameter $\eta$ ) and direct tunneling through the central region depending upon its length.

\subsection{NEGF simulation with edge effects: large scale graphene}

Until now, we have presented $I_{D}-V_{G}$ calculation from analytical formalism which ignores the effect of edge reflection. A detailed discussion was presented in Chapter 2 on this subject. The experimental observation and theoretical fit summarizes that edge effects may not be a dominant factor, since a number of physical processes such as edge roughness, incoherent scattering at the edges can in fact dilute the effect of edge effects. However, it is worth investigating how much the specular edge reflections would affect device performance. In this section, we perform NEGF simulation of the device for large width $(>500 \mathrm{~nm}$ and up to 2 $\mu \mathrm{m})$. To be able to go to such large width, we used a k.p Hamiltonian representation of the Hamiltonian instead of atomistic Hamiltonian. We have verified that such representation is as accurate as the atomistic model up to relevant energy range for most cases $(>0.6 \mathrm{eV})$. 


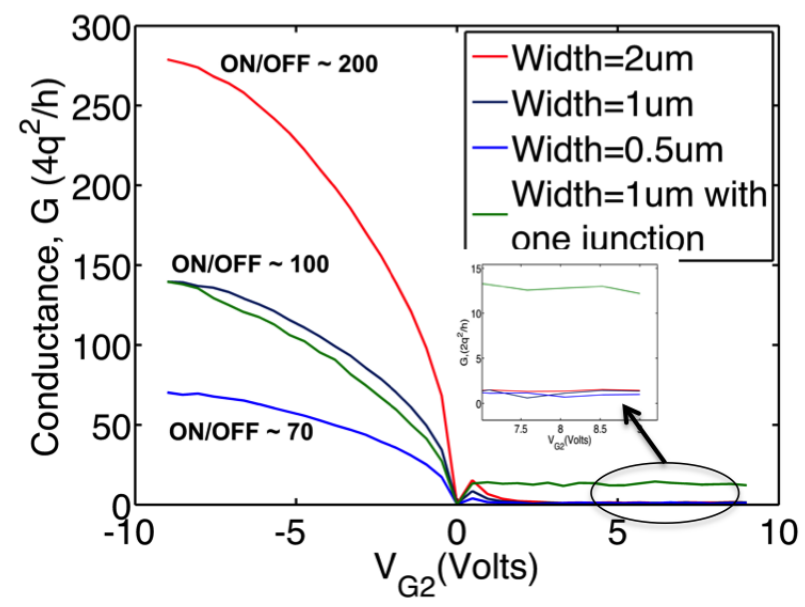

Figure 3.5: Simulation of multiple GPNJ FET for large width. ON-OFF ratio improves with width while the smaller structures show degradation of performance due to edge effects.

A hard wall boundary condition is used at the edges so that we have specular edge reflections. Fig. 3.5 shows the low bias transfer curve for several widths. As we go to larger width, the ON conductance (nnn regime) increases linearly, but the OFF conductance ( $n p n$ regime) is weakly coupled with width and remains pinned around $q^{2} / h$. Thus the ON-OFF ratio increases with width while the smaller structures show significant degradation of the ON-OFF ratio due to edge effects.

\subsection{NEGF simulation without edge effects: smaller struc- tures with open boundary condition}

To verify whether the edge effects are indeed the reason for the degradation of performance, we next show simulation results for confined structure (graphene nano-ribbons) of width $\sim$ 30nm, this time with open boundary condition.

We include two extra transverse contacts in the system to apply open boundary condition. Since this becomes a multi-terminal system and we are only interested in current from source to drain, we apply the Büttiker formalism to calculate the source-drain current. With the open boundary condition (no edge effects), the ON-OFF ratio is again quite high (well over 


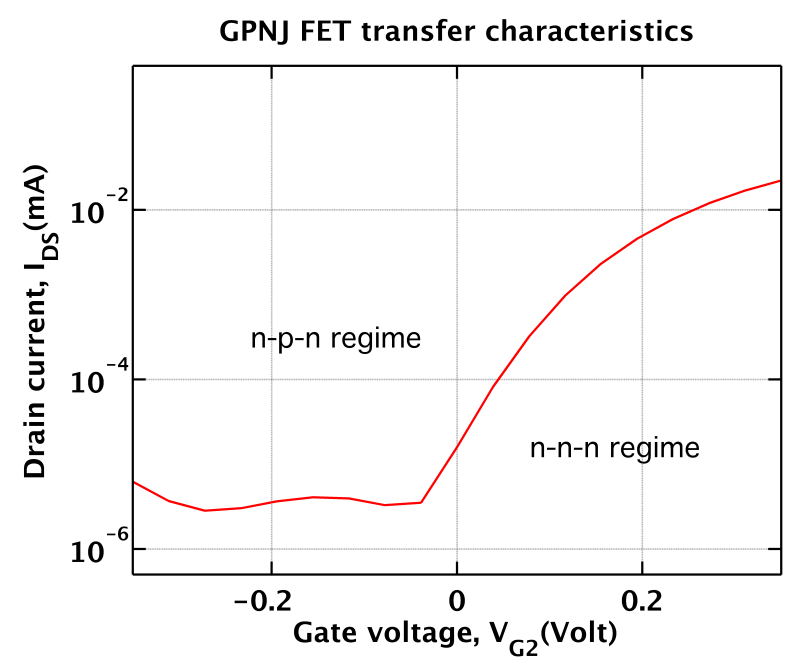

Figure 3.6: Simulation of multiple GPNJ FET for small width but with open boundary condition at the edges. ON-OFF ratio is back up to $10^{3}$.

$\left.10^{3}\right)$ at finite drain bias $\left(V_{D}=0.3 \mathrm{~V}\right)$. Such result is in agreement with the analytical formalism previously presented.

In experiments, we expect to see an ON-OFF ratio somewhere in between the two extremes (no edge effects and specular edge reflections).

\subsection{Removing Klein tunnel mode with external barrier}

In this section, we show an alternate device structure that exploits the same $p n$ junction physics. We explore a graphene junction where electrons injected by a point source are spectrally separated by a local gate, and those refracted across the junction are collected with an extended drain (Fig. 3.7). When electrons are injected from a higher electrostatically 'doped' to a lower doped side, the ones with high incident angle, low longitudinal energy are eliminated by total internal reflection. An additional tunnel barrier removes the normally incident, hotter electrons as well, resulting in a gate voltage-dependent transmission gap that allows significant gate modulation of the electron current (Figs. 3.8, 3.9). Furthermore, as we vary the gate voltage, moving progressively towards a homogenous doping across the junction, 


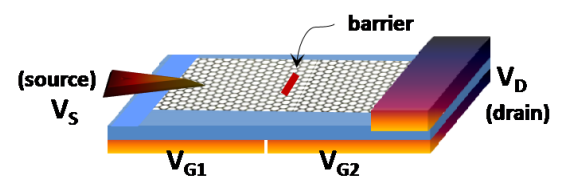

(a)

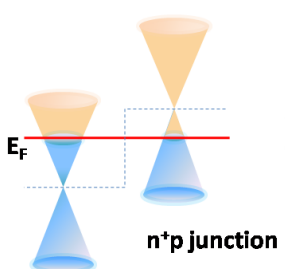

(b)

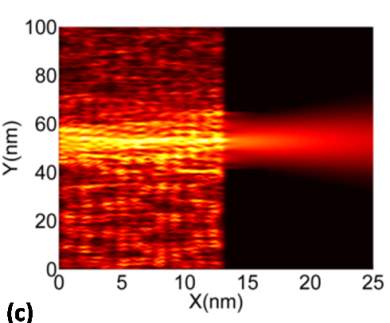

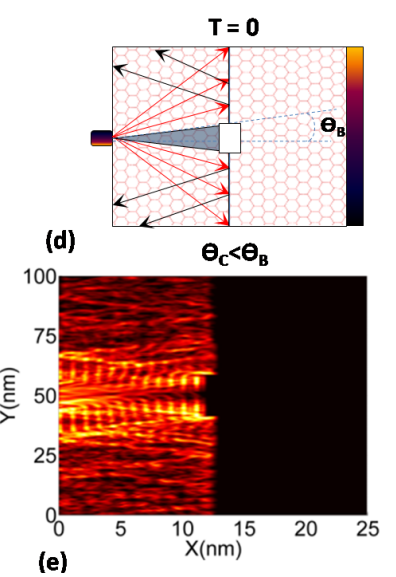

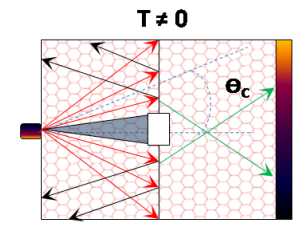

$\theta_{\mathrm{C}}>\boldsymbol{\theta}_{\mathrm{B}}$

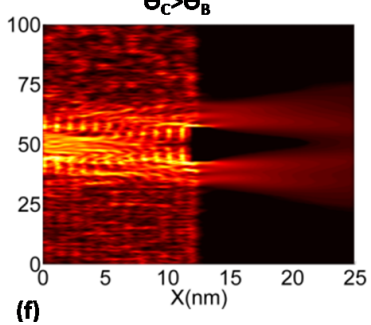

Figure 3.7: (a) Schematic diagram of the proposed device, showing a point source that angularly spreads electrons while an extended drain collects those that refract around a tunnel barrier. The point source could be lithographically patterned, an STM or electrostatically produced using analogous convex lens. Charge densities in the two segments created by the split gates are $\rho_{1}$ and $\rho_{2}$. (b) Energy band diagram for $\left|\mathrm{V}_{G 1}\right|>\left|\mathrm{V}_{G 2}\right|$. (c) With very small drain bias, atomistic current density plot calculated with Non-Equilibrium Green's function (NEGF) formalism illustrates that current flows only within the critical angle, $\theta_{C}=\sin ^{-1}\left(\left|V_{G 2} / V_{G 1}\right|\right)$. (d) Schematic Snell's Law predictions as well as (e) NEGF simulation illustrate how a barrier (void in this case) eliminates transmission within the critical angle when $\theta_{C}<\theta_{B}$, where $\theta_{B}$ is the occlusion half angle created by the barrier. (f) Transmission is non zero for $\theta_{C}>\theta_{B}$ where electrons can refract around the barrier.

we see an effective up-conversion of the local voltage, leading to a subthreshold swing lower than the thermal limit of $K T \ln 10 / q$ per decade increase of current.

Fig. 3.7a shows the schematic of the device we propose. The source injects electrons at all angles. A barrier placed at the center of the graphene sheet eliminates the normally incident electrons, so that the transmission probability with barrier can be written as

$$
T_{B}\left(\theta_{1}, E, V_{G}\right)=\Theta\left(\theta_{1}-\theta_{B}\right) T\left(\theta_{1}, E, V_{G}\right)
$$

where $\theta_{B}=\tan ^{-1} D / 2 L$ is the occlusion half angle subtended at the source by the barrier, D is length of the rectangular barrier and $\mathrm{L}$ is its perpendicular distance from the source. The barrier can be realized by a void or directed assembly of insulating molecules as demonstrated by Hersam et.al. [56]. The transmission now acts as a band-pass filter along the longitudinal energy axis $E_{L}$ (Fig. 3.8d), allowing only electrons with angles $\theta_{B}<\theta_{1}<\theta_{C}$, in other words, 


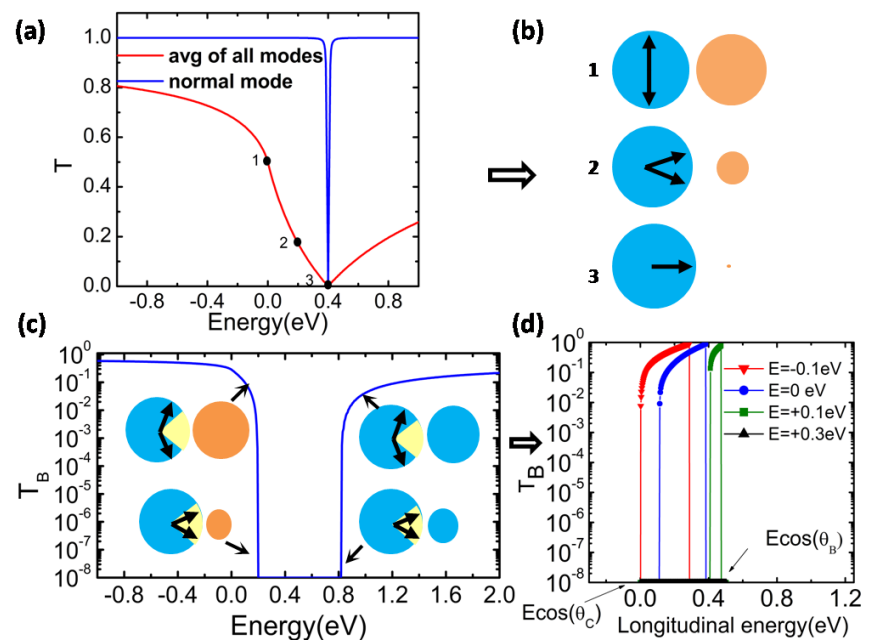

Figure 3.8: GPNJ Transmission vs. Fermi energy (E) for $V_{G 1}-V_{G 2}=+0.8 \mathrm{~V}$. Unless $\rho_{2}$ is 0 , the normal mode always transmits perfectly, making the average transmission nonzero. (b) Constant Fermi energy slices of the Dirac cones across the junction, corresponding to points on the $T(E)$ curve with arrows indicating critical angle. (c) Modification of transmission from part (a) due to the barrier, transmission within the yellow region is forbidden (d) T vs $E_{L}$ at specific total energies $E$, indicating an effective band-pass behavior for states outside transmission gap.

within a longitudinal energy window $E \cos \theta_{C}<E_{L}<E \cos \theta_{B}$ to transmit. Since no electrons can transmit when $\theta_{C}<\theta_{B}$, there is a range of energies for which we get a transmission gap (Fig. 3.8c). The creation of transmission gap is reproduced by atomistic NEGF calculation (Fig. 3.7e) with nearest neighbor $p_{z}$ orbital basis Hamiltonian. A recursive algorithm is employed to speed up the calculation of device Green's function [49] in the experimentally relevant size range $-100 \mathrm{~nm}$ wide graphene sheet containing $\sim 100,000$ atoms. We see how modes near normal incidence are reflected by the barrier, while those incident at a large angle are eliminated by total internal reflection. The gate voltage dependance of $\theta_{C}$ leads thereby to a voltage-tunable transmission gap.

We can extract an effective 'valence band-edge' $E_{V}$, given by the condition $\sin \theta_{B}=$ $\sin \theta_{C}=-\left(E_{V}+q V_{G 2}\right) /\left(E_{V}+q V_{G 1}\right)$. One can get a similar expression for the "conduction 

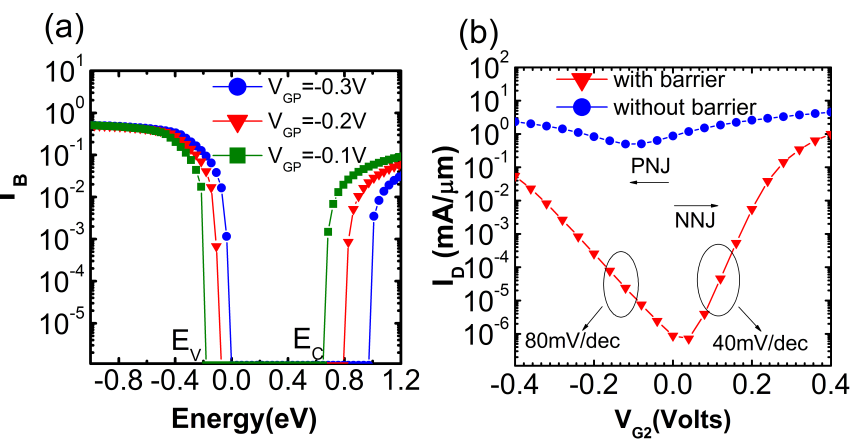

Figure 3.9: (a) Transmission shifts as we vary $V_{G 1}-V_{G 2}$, but is accompanied with a change in transmission gap (b) $\mathrm{I}_{-} \mathrm{V}_{G 2}$ of the device, showing a sharp increase in current modulation with barrier, and a subthreshold swing that is larger than the thermal limit at the PN end and smaller at the $\mathrm{N}^{+} \mathrm{N}$ end. Here, $\theta_{B}=20^{0}$ and drain bias $=0.2 \mathrm{~V}$.

band-edge' $E_{C}$, and thus the effective transmission gap $E_{G}$

$$
\begin{array}{r}
E_{V, C}=-q V_{G 2} \mp q \frac{\left(V_{G 1}-V_{G 2}\right) \sin \theta_{B}}{1 \pm \sin \theta_{B}} \\
E_{G}=E_{C}-E_{V}=q\left(V_{G 1}-V_{G 2}\right) \frac{2 \sin \theta_{B}}{\cos ^{2} \theta_{B}}
\end{array}
$$

Thus $E_{G}$ is proportional to the built-in potential, $V_{0}=q\left(V_{G 1}-V_{G 2}\right)$. There are two distinct contributions to the resulting transmission plot (Fig. 3.9a)-(i) an overall shift given by $-q V_{G 2}$, and (ii) a voltage modulation of the band-gap $E_{G}$. The first term will lead to the electrostatic gating effect seen for regular band-gapped semiconductors (this leads to $\sim 60$ $\mathrm{mV} /$ decade at room temperature), while the latter term creates a deviation from this result, effectively captured through a gate tunable, continuous metal-insulator transition associated with voltage dependence of $E_{G}$. The gap vanishes in the homogeneous doping limit $\left(V_{0}=0\right)$, requiring the 'valence band-edge' to slow down while the 'conduction band-edge' to catch up.

Fig. 3.9b shows the corresponding current extracted using the Landauer formalism for various gate voltages $V_{G 2}$ while $V_{G 1}$ is kept fixed. While the $\mathrm{ON}$ current reduces a little, the tunnel barrier primarily reduces the OFF current by nearly five orders of magnitude. Overall, an ON-OFF ratio of $\sim 10^{4}$ is achievable with just $0.25 \mathrm{~V}$ change in gate voltage on the $\mathrm{nn}$ 
regime with $\theta_{B}$ of $20^{\circ}$. This will require a $70 \mathrm{~nm}$ long barrier for $1 \mu \mathrm{m} \times 1 \mu \mathrm{m}$ graphene sheet, if the source is placed at $200 \mathrm{~nm}$ from the $p n$ interface. The unusual transconductance and subthreshold swing can be attributed to the fact that transmission gap changes every time the gate voltage $V_{G 2}$ is changed. From Eq. 3.12 the subthreshold swing can be calculated as,

$$
S=\left(d \log _{10} I / d V_{G 2}\right)^{-1}=K T \ln 10\left[1 \mp \sin \theta_{B}\right] / q
$$

which is less than $60 \mathrm{mV} / \mathrm{dec}$ ('-' sign above) at the $n n$ end, and more than $60 \mathrm{mV} / \mathrm{dec}($ '+' sign) at the $n p$ end. At heart of the unusual behavior is the coupling of angular and energy filtering in the barrier driven Klein tunnel-switch, which differs from most materials in that its effective band-gap can be collapsed with a gate voltage.

To summarize this section, we have shown that the physics of regular tunneling coupled with Klein tunneling opens up the opportunity for high performance low power switching based on graphene $p n$ junction. The additional barrier creates a transmission gap and increases the ON-OFF ratio substantially by removing the high longitudinal energy electrons which are responsible for high OFF current. The gate voltage dependence of the transmission gap leads to overcoming thermal switching limit of $K T \ln 10 / q$ per decade. Therefore, despite being different architecture, the two devices shown in this chapter in essence produce very similar result, driven the filtering of pseudospins across pn junctions.

This chapter thus provides two different device designs that aim to eliminate the problem of high leakage current in graphene by filtering out both high and low angle modes either by multiple pn junctions or external barrier. Such devices yield high ON-OFF ratio without the need of a bandgap but with a gate tunable transmission gap yielding sub-thermal switching. Next chapter discusses how we can use such devices as building blocks of novel electronic and spintronic systems.

\section{Related publications:}

1. Redwan N. Sajjad, Avik Ghosh, "Manipulating Chiral Transmission by Gate Geometry: 
Chapter 3 | Transmission gap: a new way of switching

Switching in Graphene with Transmission Gaps", ACS Nano, vol. 7, no. 11, pp. 9808 (2013).

2. Redwan N. Sajjad, Avik Ghosh, "High efficiency switching using graphene based electron “optics"', Applied Physics Letters vol. 99, pp. 123101(2011). 


\section{Chapter 4}

\section{What we can do with pseudospin(spin) filtering by $p n$ junction}

In previous chapters, we showed how gate modulation of current is possible in graphene $p n$ junction devices. In this chapter we show how this can be useful as a logic device ${ }^{1}$. In the second segment of this chapter, we discuss how we can modulate spin current (instead of pseudo-spins) in topological insulators (TI $)^{2}$ based $p n$ junction with implications in spintronic devices.

\subsection{GPNJ as a Boolean logic device}

A. Gate-Level Model: In this section, we present the models for GPNJ based inverter and NAND gates, which we use for evaluating the circuit level performance.

Inverter: A GPNJ inverter is composed by two GPNJ switches that share one contact in the middle of the inverter as the output, shown in Fig. 4.1. Green and yellow gates are applied with positive and negative control voltages. The input A is connected to two trapezoid top gates that can turn on either side of the GPNJ switch depending on the input. If the input

\footnotetext{
${ }^{1}$ This work was done in collaboration with Chenyun Pan and Azad Naeemi, ECE, Georgia Tech.

${ }^{2}$ This part of the work was done in collaboration with K. M. Masum Habib, ECE, UVa
} 

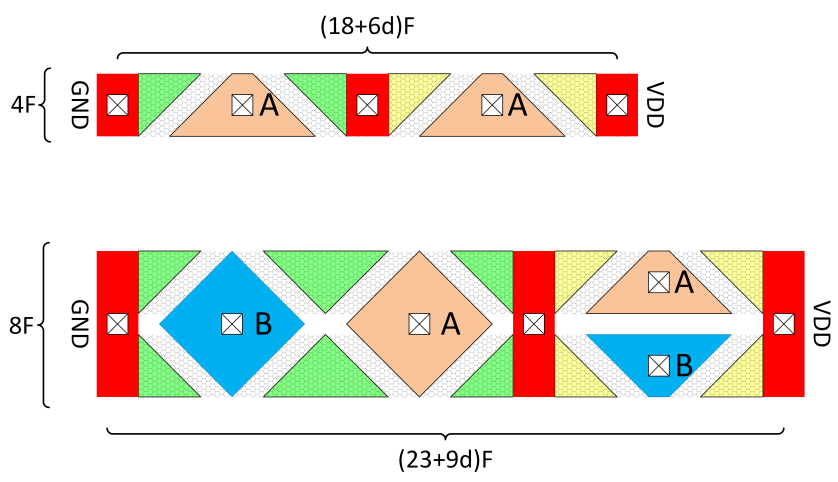

Figure 4.1: Gate-Level GPNJ layout: (a) Inverter (b) NAND gate. Here, D is the gap distance of the GPNJ junction, and F is the minimum feature size, which is $16 \mathrm{~nm}$ in this work.

is logic ' 1 ', the output will be connected with the bottom ' 0 '; if the input is logic ' 0 ', the output will be connected with the top logic '1'. In Fig. 4.1a, the footprint area model for a GPNJ inverter is depicted to be compatible with the standard CMOS process, the design rules such as the minimum spacing between two nearby vias follow from Ref. [57].

NAND Gate: The layout for a NAND gate is shown in Fig. 4.1b, where $20 \%$ of the footprint area reduction can be achieved by using the chemical doping control method. The resistance and capacitance values for a GPNJ NAND gate can also be obtained based on the width of the graphene sheet in the pull-up and pull-down network.

TABLE I presents circuit-level metrics for GPNJ- and CMOS-based logic devices and circuits. The $16 \mathrm{~nm}$ CMOS numbers are from the Predictive Technology Model (PTM) [58]. In terms of delay, a GPNJ inverter provides a faster intrinsic speed due to its smaller output resistance. The larger gate capacitance of the GPNJ inverter is caused by the large input trapezoid gates. For a 32-bit adder, the GPNJ circuit can offer about 70\% delay and energy-delay-product (EDP) reduction due to its excellent current driving capability compared to their Si CMOS counterpart. Additionally, the smaller footprint area of the GPNJ devices can shorten the interconnect length. Therefore, it reduces the parasitic capacitance and dynamic power dissipation for wires, which contributes to the speed improvement of the circuit. 
TABLE I

CirCUIT-LEVEl COMPARISON OF VARIOUS PERFoRMANCE METRICS BETWEEN

SI CMOS CIRCUITS AND GPNJ CIRCUITS

\begin{tabular}{|c|c|c|c|}
\hline \multicolumn{2}{|r|}{ Metrics } & CMOS & GPNJ \\
\hline \multirow{4}{*}{ INV } & Intrinsic Speed (ps) & 1.42 & 1.31 \\
\hline & Switching Energy (aJ) & 13.50 & 26.52 \\
\hline & Static Power $(\mathrm{nW})$ & 7.41 & 6.73 \\
\hline & $\operatorname{Area}\left(\mathrm{F}^{2}\right)$ & 160 & 120 \\
\hline \multirow{4}{*}{ NAND } & Intrinsic Speed (ps) & 4.35 & 1.47 \\
\hline & Switching Energy (aJ) & 18.00 & 39.78 \\
\hline & Static Power $(\mathrm{nW})$ & 7.76 & 10.10 \\
\hline & $\operatorname{Area}\left(\mathrm{F}^{2}\right)$ & 336 & 328 \\
\hline \multirow{6}{*}{$\begin{array}{l}\text { 32-bit } \\
\text { Adder }\end{array}$} & Delay (ps) & 214.94 & 59.91 \\
\hline & Dynamic Power $(\mu \mathrm{W} / \mathrm{GHz})$ & 3.41 & 4.62 \\
\hline & Energy (fJ) & 4.54 & 4.99 \\
\hline & $\begin{array}{l}\text { Energy-Delay Product } \\
\text { (fJ*ps) }\end{array}$ & 975.36 & 298.94 \\
\hline & Static Power $(\mu \mathrm{W})$ & 5.26 & 6.19 \\
\hline & Area $\left(\mu \mathrm{m}^{2}\right)$ & 65.27 & 60.16 \\
\hline
\end{tabular}

Figure 4.2: The delay and power dissipation components of GPNJ logic circuits for inverter, NAND and adder.

For the same feature size $(\mathrm{F}=16 \mathrm{~nm})$, the GPNJ logic is always faster than CMOS logic, due to its high $\mathrm{ON}$ current. The difference goes up as we go into larger systems: from inverter to NAND and Adder, where higher number of devices are connected in series. For CMOS, the series resistance goes up but for GPNJ, it stays the same due to its ballistic nature of electron transport. The switching are for GPNJ is a bit larger due to its angled gates. This results in a higher switching energy $\left(\frac{1}{2} C_{G} V_{D D}^{2} f\right)$, but the interconnect lengths are determined by the total area, where GPNJ has advantage over CMOS. Both the interconnect lengths and lower output resistance play role in lowering delay. In the end, the energy delay product for GPNJ is much lower (298.94 fJ-ps) compared to the CMOS counterpart (975.36 fJ-ps).

\subsection{Spin filtering in topological insulators}

In this section, we show that the conservation of momentum of massless, Dirac-Fermionic surface states of 3D topological insulator pn junction leads to spin filtering effect analogous to the pseudospin filtering in graphene. Due to spin-momentum locking in topological insulator, 

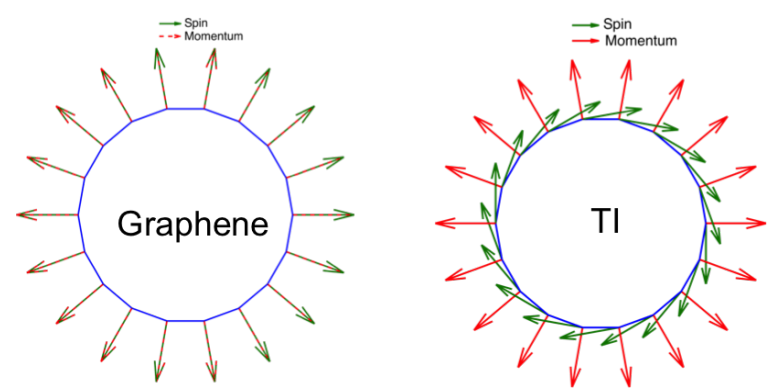

Figure 4.3: spin (pseudospin) locking in graphene (a) and topological insulator (b).

the spin of electron is flipped when it is reflected back from the pn junction interface which enhances the spin current at the source contact. As a result, the spin current increases and the charge current decreases leading to extremely high spin Hall angle (up to 20). Moreover, both the spin current and the spin Hall angle are gate tunable which makes the Topological Insulator pn junction a promising device in spintronics.

Topological insulator (TI) has been in the center of attention in condensed matter physics in the past decade because of its unusual electronic properties classified as a new state of matter [59]. The spin orbit coupling leads to a bulk bandgap in this class of materials and at the same time exhibits gapless edge (for 2D) and surface (for 3D) states protected by time reversal symmetry. TI's are theoretically predicted and experimentally verified in quantum wells [60] and bulk crystals [17, 61]. The low energy effective Hamiltonian to describe the surface states of TI has been shown to be $H=v_{F} \hat{z} \cdot(\boldsymbol{\sigma} \times \mathbf{p})$ by fitting the ab initio calculations in Ref. [17]. The Hamiltonian is much similar to that of graphene Hamiltonian at low energy given by $H=v_{F} \boldsymbol{\sigma} \cdot \mathbf{p}$ with the exception that for TI, the Pauli matrices $\sigma$ operate on the real spins while for graphene it operate on pseudospins representing the A-B sublattices. In both systems, the spin (or pseudospin) is locked with the momentum, for TI they are at $90^{\circ}$ to each other and for graphene they are in parallel as shown in Fig. 4.3. The spin-momentum locking can have important implication in spintronics applications where a highly spin polarized current is required to switch a magnet with as low charge current as possible to reduce the Ohmic dissipation. Magnetic tunnel junction (MTJ) has been used for Spin -transfer Torque devices to perform basic read and write operation with spin degree of freedom [62, 63]. A 


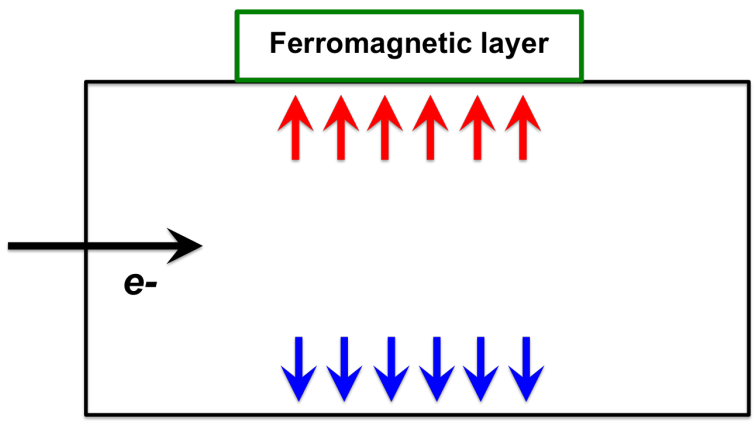

Figure 4.4: Spin Hall effect, where the charge current is accompanied with a transverse spin current.

free layer (where the data is to be kept) is separated from a fixed reference magnet by a tunneling barrier. A non-polarized current is passed through the fixed layer of magnet, gets spin polarized and performs spin torque (write) if the spin current exceeds a critical limit. A smaller current in the device will not produce spin switching, but we can read the spin state from the resulting charge current in the device. A parallel spin configuration will produce a lower resistance, whereas an anti-parallel configuration produces a higher resistance (read).

It has been shown theoretically in the past $[64,65]$ that a charge current is always accompanied with a spin current transverse to the direction of charge current in materials with large spin orbit coupling. Opposite spins accumulate in either side of the sample just like Hall effect, where opposite charges accumulate on opposite sides. But in this case the spins accumulate without the assistance of a magnetic field and thus have potential device applications. Fig. 4.4 shows the spin accumulation and how it can switch the ferromagnetic layer (on top of the original material) by hitting the magnet with spins. Recently, the discovery of Giant Spin Hall Effect (GSHE) has been shown to generate sufficient spin current to spin-torque switch a ferromagnet at room temperature[66, 67] and logic schemes based on GSHE have been proposed [63], but the problem of high charge dissipation persists. The key parameter to describe the effectiveness of a material for spintronic applications is the spin Hall angle $\theta_{H}=\frac{2 I_{s} / \hbar}{I_{q} / q}$ where $I_{s}$ and $I_{q}$ are the spin and charge currents respectively. Spin Hall angles for various metals and metal alloys has been found to vary between 0.07-0.3 


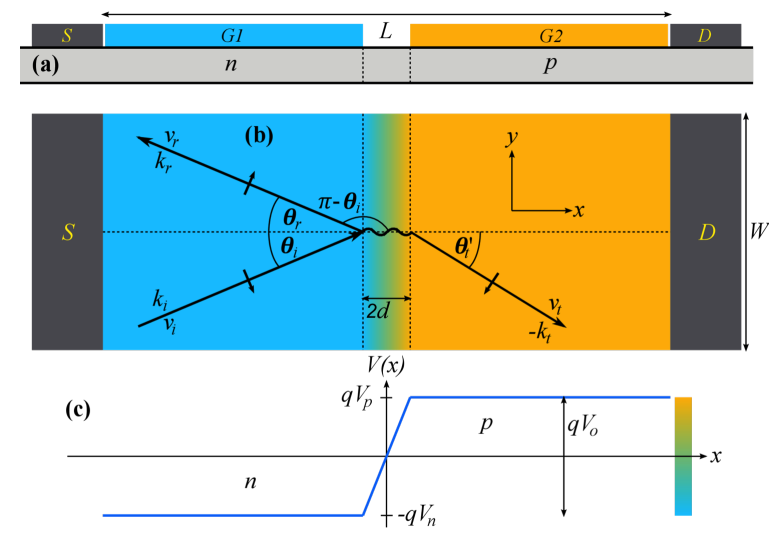

Figure 4.5: (a) Cross section and (b) top view of 3D TI pn junction with incident, reflected and transmitted electron waves. The long arrows indicate the direction of group velocity and wavevector at a given energy $-q V_{n}<E<q V_{p}$. The short arrows indicate the direction of spin. (c) Potential profile of the device. For simplicity, linear potential profile is assumed between $n$ and $p$ regions.

$[68,69,66,70,71,72,67]$. Recently, spin hall measurements on $\mathrm{Bi}_{2} \mathrm{Se}_{3}$ based TI has been reported to have $\theta_{H}=2-3.5[73]$.

In recent years, the transport physics of graphene based $p n$ junction has been studied both theoretically $[37,43,74,75,54,55]$ and experimentally $[41,45,2,76]$ because of its unique tunneling characteristics. As we described earlier, the tunneling across the junction demonstrates an angle (transverse mode) dependent transmission probability (perfect at normal incidence called Klein tunneling) [37] originating from the chiral nature of graphene electrons. With a split $p n$ junctions, the junction acts as a filter for psuedospins and only allows very low angle electrons to pass through [43]. The similarity of the Hamiltonian of graphene and the surface of 3D TI suggests that similar filtering of real spins should occur in TI pn junction.

Momentum filtering in spit gate TI pn junction (Fig. 4.5) only allows electrons with very small incident angles to pass through and all other electrons are reflected back to the source [77]. As a result, charge current going through the junction decreases. Due to spin-momentum locking, the injected electrons have down spin but the reflected electrons have up spin which enhances the spin current at the source contact. This results in an extraordinarily large 


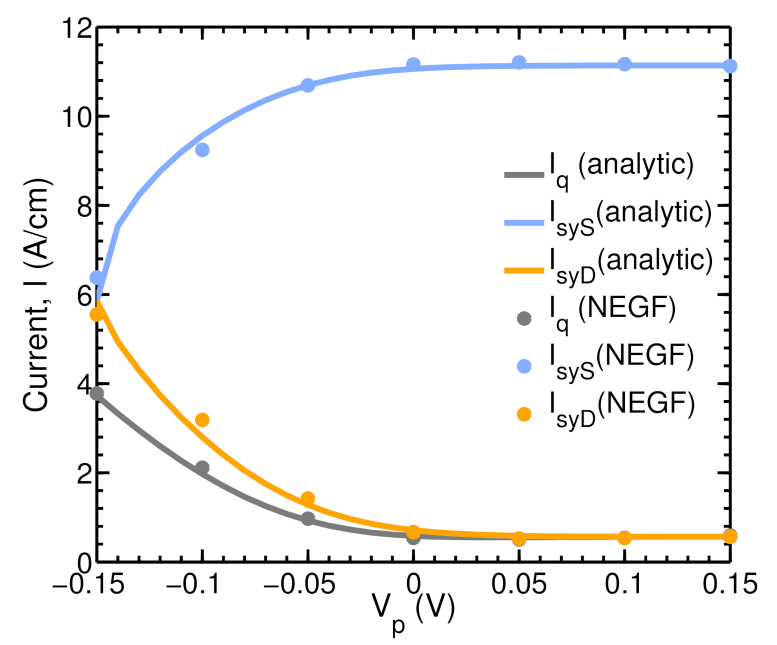

Figure 4.6: Normalized charge and spin current as functions of gate voltage $V_{p}$ for a device with width $W=100 \mathrm{~nm}$, length $L=120 \mathrm{~nm}$ and split length $2 d=100 \mathrm{~nm}$, drain bias $V_{D}=0.1 \mathrm{~V}$ and gate voltage $V_{n}=0.15 \mathrm{~V}$. Thus, built in potential $V_{0}=0.3 \mathrm{eV}$ when $V_{p}=0.15$ $\mathrm{V}$.

spin to charge current ratio (can be called longitudinal spin Hall angle). We find that in a split-gate, symmetrically doped TI $p n$ junction, the spin Hall angle at the source contact is (see Appendix $\mathrm{C}$ for detail derivation),

$$
\theta_{H} \approx \pi \sqrt{\frac{2 d V_{0}}{\hbar v_{F}}}-1
$$

$V_{0}$ is the built in potential of the $p n$ junction and $2 d$ is the spit between the gates. For a device with $2 d=100 \mathrm{~nm}$ and $V_{0}=0.3 \mathrm{eV}$, the spin Hall angle is $\sim 20$.

The top view of the model $p n$ junction device is shown in Fig. 4.5a. The source $(\mathrm{S})$ and the drain (D) contact are placed on the top surface of a 3D TI slab. Electrons are injected from source and collected at drain by a bias voltage $V_{D}$. The $p$ and $n$ regions are electrically doped using two external gates separated by the split distance $d$. The device has a built-in potential $V_{0}=V_{p}+V_{n}$ distributed between the $p$ and $n$ regions such that the electrostatic potential energy, $V(x)=-q V_{n}, q\left[\frac{V_{0}}{d} x+\frac{V_{0}}{2}-V_{n}\right]$ and $-q V_{p}$ for $x<d,-d<x<d$ and $x>d$ respectively as shown in Fig. 4.5 c.

Fig. 4.6 shows the charge and spin current vs. gate bias of the $p$ region calculated using 


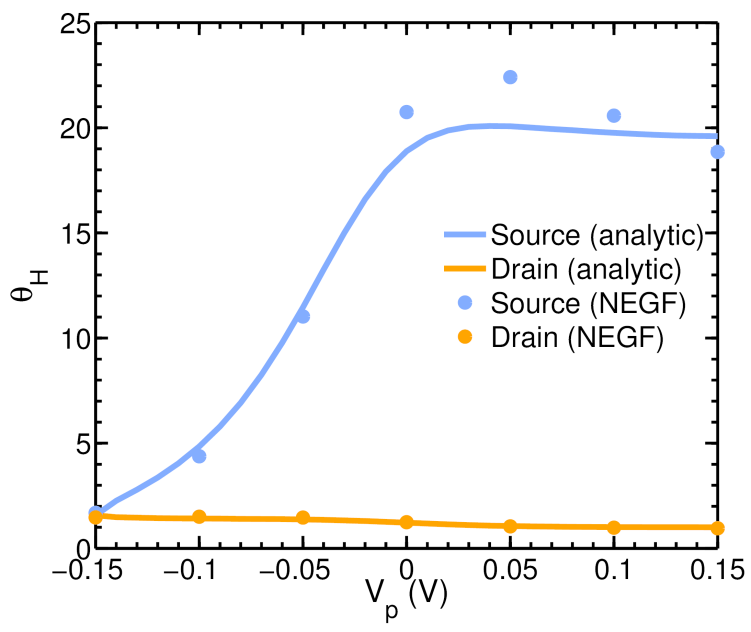

Figure 4.7: Spin Hall angle $\theta_{H}$ as a function of $V_{p}$.

Eq. C.23 for a device with length $L=120 \mathrm{~nm}$, width $W=100 \mathrm{~nm}$, split length $2 d=100$ nm, the drain bias $V_{D}=0.1 \mathrm{~V}$ and gate voltage $V_{n}=0.15 \mathrm{~V}$. When the gate voltage of $p$ region $V_{p}=-0.15 \mathrm{~V}$, the channel is a uniform $n n$ material with uniform potential profile. Thus, all the modes are allowed to transmit from the source to the drain and there is no reflection. Hence, the charge current is maximum, spin current at the source and drain are equal and $\theta_{S}=\pi / 2$ as shown in Fig. 4.7. When the gate voltage $V_{p}$ is increased, the potential profile is no longer uniform, the channel becomes a $n-n^{+}$junction and some of the electrons are reflected back from the junction. As a result, the charge current and the spin current at drain decreases and the spin current at source decreases. Therefore, the spin Hall angle changes from 1.5 to 20 at source contact and 1.5 to 1 at the drain when the gate voltage $V_{p}$ changes from $-0.15 \mathrm{~V}$ to $0.15 \mathrm{~V}$. This is by far the highest spin Hall angle reported in the literature. This may have application in spin based logic and memory devices, which require spin polarized current to switch magnetic materials with as low charge current as possible to reduce power dissipation.

\section{Related publications:}

1. K. M. Masum Habib, Redwan N. Sajjad, Avik Ghosh, "Chiral tunneling of topological states for giant longitudinal spin Hall angle", arxiv:1402:6222v1 (2014). 


\section{Chapter 5}

\section{Non-Equilibrium Green's Function (NEGF) formalism: a rigorous transport model}

So far, we presented numerical results along with analytical results throughout the dissertation. In this chapter, we describe the details of the NEGF based numerical model and show its usefulness to simulate devices for not only quantum effects, but also diffusive transport as well. We already presented results for GPNJ devices. We now present electron, spin and magneto-transport for regular graphene devices (no junction) in this chapter.

\subsection{NEGF: an efficient Schrödinger solver}

An electronic system in the quantum regime is described by Schrödinger equation,

$$
E \Psi=[H] \Psi
$$

This is the time independent version where $[H]$ is the Hamiltonian of the system containing the information of the energy levels. $\Psi$ and $E$ are the eigenfunction and eigenvalue respectively. 
Chapter 5 | Non-Equilibrium Green's Function (NEGF) formalism: a rigorous transport model58

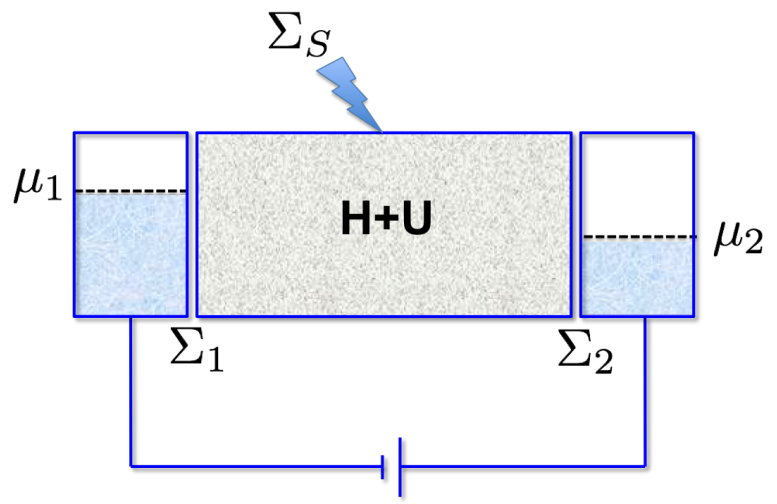

Figure 5.1: Device Hamiltonian $[H]$, contact information (self-energy $[\Sigma]$ and electro-chemical potentials $\mu_{1,2}$ ), device potential $U$ and scattering self-energy $\left[\Sigma_{S}\right]$ go as inputs to NEGF formalism and it provides the transport properties such as carrier density and terminal current.)

We argue that the system described by Eq. 5.1 is that of an isolated system, without any contact with the outside world. The solution of the time dependent Schrödinger equation describes how the electron evolves in time and space,

$$
\begin{aligned}
i \hbar \frac{\partial \Psi}{\partial t} & =H \Psi \\
\Psi(\mathbf{r}, \mathbf{t}) & =\Psi(r) e^{-i E t / \hbar}
\end{aligned}
$$

thus $\frac{\partial\left(\Psi \Psi^{\dagger}\right)}{\partial t}=0$, meaning the electron will always stay in the eigenstate without any decay, since the system is isolated (infinite lifetime $\tau->\infty$ ). Practical electronic devices are almost always connected to two contacts (may be called source and drain). As shown in Fig. 5.1, the channel is connected to two contacts and the Fermi energies are at $\mu_{1}$ and $\mu_{2}$ defined by the intrinsic Fermi energy and the applied drain bias. The effects of the contacts are to be incorporated through a correction to the Hamiltonian, called self energy, $[\Sigma]$ matrices.

\subsubsection{Modified Schrödinger equation}

The Schrödinger equation can be modified for the above system can be written as,

$$
E\{\Psi\}=\left[H+\Sigma_{1}+\Sigma_{2}\right]\{\Psi\}+\{S\}
$$


The effect of the contacts are incorporated in two ways, one is the outflow of electrons into the contact $([\Sigma]\{\Psi\}$ term) and the other is the inflow $\{S\}$ term which injects electrons into the channel from contact. The aim is to solve the modified Schrödinger equation Eq. 5.4 to get the relevant device quantities such as electron density $G^{n}=\{\Psi\}\{\Psi\}^{\dagger}$ and terminal current $I_{1,2}$. Non-zero channel potential $U$ can be added to $[H]$ and any form of scattering (either elastic or inelastic) are to be incorporated with either $U$ or additional self energies $\left[\Sigma_{S}\right]$. Let us now limit our discussion in the absence of $U$ and scatterings.

From Eq. 5.4, $\{\Psi\}$ can be written as,

$$
\{\Psi\}=[E \mathbb{I}-H-\Sigma]^{-1}\{S\}
$$

where $\Sigma=\Sigma_{1}+\Sigma_{2}$. Let us define the retarded Green's function,

$$
G^{R}=[E \mathbb{I}-H-\Sigma]^{-1}
$$

where $\mathbb{I}$ is the identity matrix with dimension equal to that of $[H]$. From Eq. 5.5, the $G^{R}$ is the solution of the system under impulse input.

To get the carrier density, we can write

$$
\{\Psi\}\{\Psi\}^{\dagger}=G^{R}\{S\}\{S\}^{\dagger} G^{A}
$$

We next show how to the strength of the source $\{S\}\{S\}^{\dagger}$ depends on the self energy matrix.

\subsubsection{Broadened density of states and electron escape rate}

We started the section by showing how an electron lifetime is infinite for an isolated system. For the system with contacts, it is no longer isolated and the electron density with time should change. 
Chapter 5 Non-Equilibrium Green's Function (NEGF) formalism: a rigorous transport model60

Let us assume that $\Sigma=i \frac{\gamma}{2}$. We will later see that the quantity $\gamma$ (the anti-Hermitian component of $\sigma$ from the definition) is the escape rate $(\hbar / \tau)$ of electrons and therefore decides the broadening of energy levels. We have

$$
\frac{\partial\left(\Psi \Psi^{\dagger}\right)}{\partial t}=e^{i\left(E-i \frac{\gamma}{2}\right) t / \hbar-i\left(E-i \frac{\gamma}{2}\right) t / \hbar}=e^{-\gamma t / \hbar}
$$

thus the self-energy correction to the Hamiltonian makes electrons have a decay rate determined by $\gamma$. This makes sense since electron in the channel connected to an open system will leak into the contact and decay gradually until $\Psi$ is 0 . For a single level channel (eigenvalue at $\epsilon$ ) and using $\psi=G^{R}$ s, we can write for density of states $(D)$,

$$
\begin{array}{r}
D=\Psi \Psi^{\dagger}=\frac{s s^{*}}{(E-\epsilon)^{2}+(\gamma / 2)^{2}} \\
D=\frac{\gamma / 2 \pi}{(E-\epsilon)^{2}+(\gamma / 2)^{2}}
\end{array}
$$

which has a broadening proportional to $\gamma$. Since the energy level broadening is related to the escape rate (inverse lifetime) through uncertainty relation, it makes sense to define the broadening as $\gamma=\frac{\hbar}{\tau}$ where $\tau$ is the lifetime. It can be shown [78] that the strength of the source $s s^{*}$ is related to the energy broadening,

$$
2 \pi s s^{*}=\gamma f
$$

Using this in Eq. 5.7 we get for Carrier density,

$$
G^{n}=G^{R} \Sigma^{i n} G^{A}
$$

where $\left[\Sigma^{i n}\right]=[\Gamma] f$, where $f$ is the Fermi function. Some variable above are used in lowercase (such as $\gamma$ ) when working with a one level system. The capital letter variables signify the matrix version of the same quantities for a multi-level system. 


\subsubsection{Current and transmission: Landauer formula}

Current at one terminal is given by $\frac{d N}{d t}$. To find this, we go back to time dependent Schrödinger equation,

$$
\begin{array}{r}
i \hbar \frac{d\{\Psi\}}{d t}=[H+\Sigma]\{\Psi\}+\{S\} \\
-i \hbar \frac{d\{\Psi\}^{\dagger}}{d t}=\{\Psi\}^{\dagger}\left[H+\Sigma^{\dagger}\right]+\{S\}^{\dagger}
\end{array}
$$

By using $N=\{\Psi\}\{\Psi\}^{\dagger}$ and $\{\Psi\}=G^{R}\{S\}$, it can be shown [78] current at terminal $m$,

$$
I_{m}(E)=\frac{q}{h} \operatorname{Trace}\left[\Sigma_{m}^{i n} A-\Gamma_{m} G^{n}\right]
$$

Using $G^{n}=G^{R} \sum^{i n} G^{A}$ and $A=i\left(G^{R}-G^{A}\right)$, Eq. 5.13 can be reduced to,

$$
\tilde{I_{m}}(E)=\frac{q}{h} \operatorname{Trace}\left[\Gamma_{1} G^{R} \Gamma_{2} G^{A}\right]\left(f_{1}(E)-f_{2}(E)\right)
$$

The term within square bracket is Conductance $(G)$ or total Transmission $(T)$

$$
G=\frac{q^{2}}{h} \operatorname{Trace}\left[\Gamma_{1} G^{R} \Gamma_{2} G^{A}\right]
$$

at zero temperature. Eq. 5.14 shows energy resolved current. Total terminal current,

$$
I_{m}=\frac{q}{h} \int T(E)\left[f_{1}(E)-f_{2}(E)\right] d E
$$

The finite temperature conductance can be deduced from Eq. 5.16. For small bias $f_{1}(E)-$ $f_{2}(E)$ can be Taylor expanded and with just the leading term, we get

$$
\begin{aligned}
I_{m} & =\frac{q}{h} \int G(E)\left(-\frac{\partial f}{\partial E}\right)\left(\mu_{1}-\mu_{2}\right) d E \\
I_{m} & =\frac{q}{h}\left(\mu_{1}-\mu_{2}\right) \int\left(-\frac{\partial f}{\partial E}\right) G(E) d E
\end{aligned}
$$


Chapter 5 Non-Equilibrium Green's Function (NEGF) formalism: a rigorous transport model62

Giving us the conductance $G\left(E_{f}\right)=I_{m} / V_{D}$,

$$
G\left(E_{f}\right)=\frac{q^{2}}{h} \int\left(-\frac{\partial f}{\partial E}\right) G(E) d E
$$

using $\mu_{1}-\mu_{2}=q \cdot V_{D}$. At zero temperature this can be written as,

$$
G\left(E_{f}\right)=\frac{q^{2}}{h} \sum_{n} T_{n}
$$

The total conductance is the sum of transmissions from all transverse modes. Eq. 5.20 is known as the Landauer formula, named after Ralf Landauer who first explained current in terms of transmission probability of electrons [79].

This concludes our discussion on NEGF and how to calculate relevant device quantities given $[H]$ and $[\Sigma]$. We next show simulation results of graphene using this approach for various aspects of transport.

\subsection{Ballistic electron transport in graphene}

\subsubsection{Electronic conductance}

We perform the NEGF simulation to calculate the density of states (DOS) and conductance of graphene sheets of various widths $(W)$. Fig. 5.2a shows the DOS (and conductance in inset) for $W=10$ and $100 \mathrm{~nm}$. Narrower nanoribbons show a gap as well as Van Hove singularities due to quantization for select chiralities while wider sheets show a quasi-linear bulk graphene density of states. In practice, nanoribbons also need attention to edge state dynamics, particularly the presence of strain and roughness [80].

We next focus on how the total conductance varies with channel doping (Fig. 5.3) with emphasis on the lowest mode in single-layer graphene (SLG) and Bilayer graphene (BLG). The expected quantum interference oscillations at low temperature and conductance asymmetry 

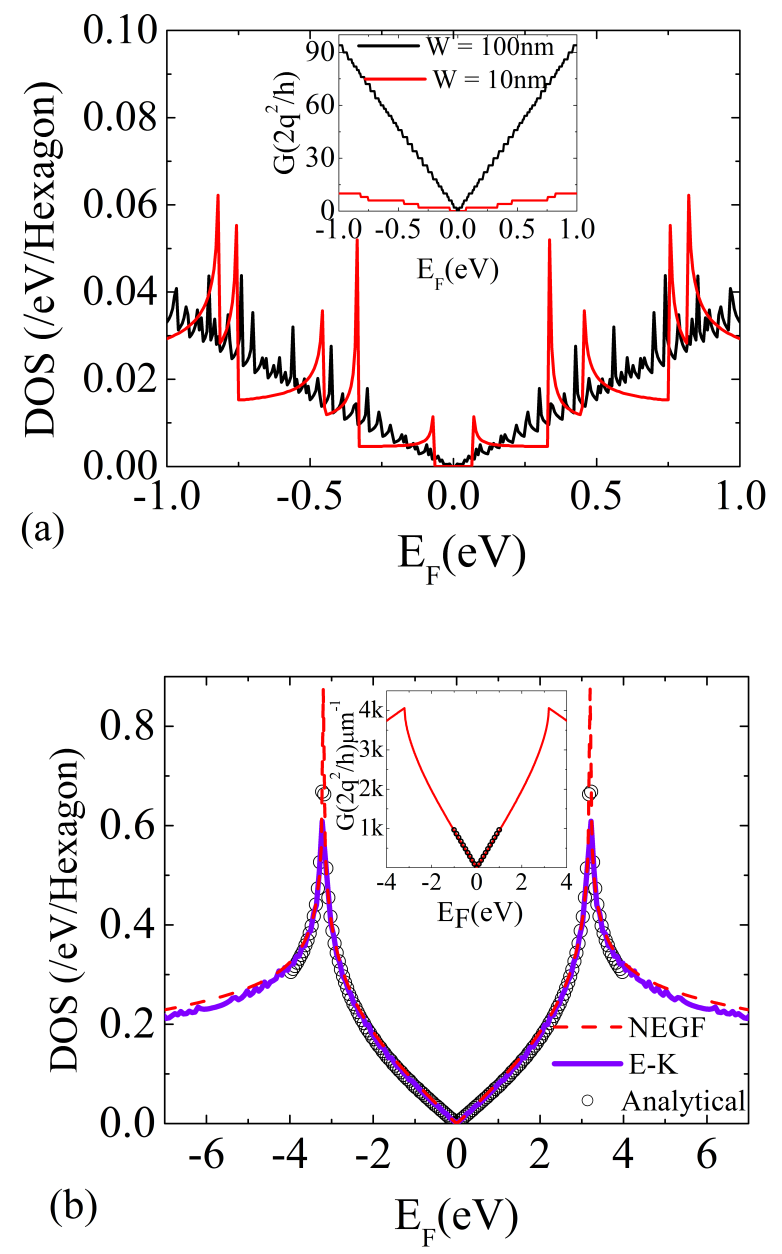

Figure 5.2: (a) Density of states (DOS) and conductance (inset) calculation from atomistic tight binding RGFA for two different widths, (b) Similar calculations over a wider energy range done with $\mathrm{KSF}$, an integration of the $E-k$ dispersion and from analytical expression in Ref. [3]. Conductance from KSF (red line, inset) matches with simple linear approximation of no. of modes (black circles) at low energy.

are seen due to formation of Fabry-Pérot cavity from non-uniform dopings. The lowest mode show almost no oscillation for SLG while the BLG shows oscillations just like the higher order modes. The Fabry-Pérot oscillations originate primarily from non perfect transmission and the subsequent reflection and transmission within the cavity. The lowest mode in single layer graphene has linear E-K with near perfect transmission through the heterojunction due to Klein tunneling, making the oscillations negligible. For the bilayer graphene, the parabolic nature of the lowest mode leads to non-unity transmission giving rise to the oscillation. Such 
Chapter 5 | Non-Equilibrium Green's Function (NEGF) formalism: a rigorous transport model64

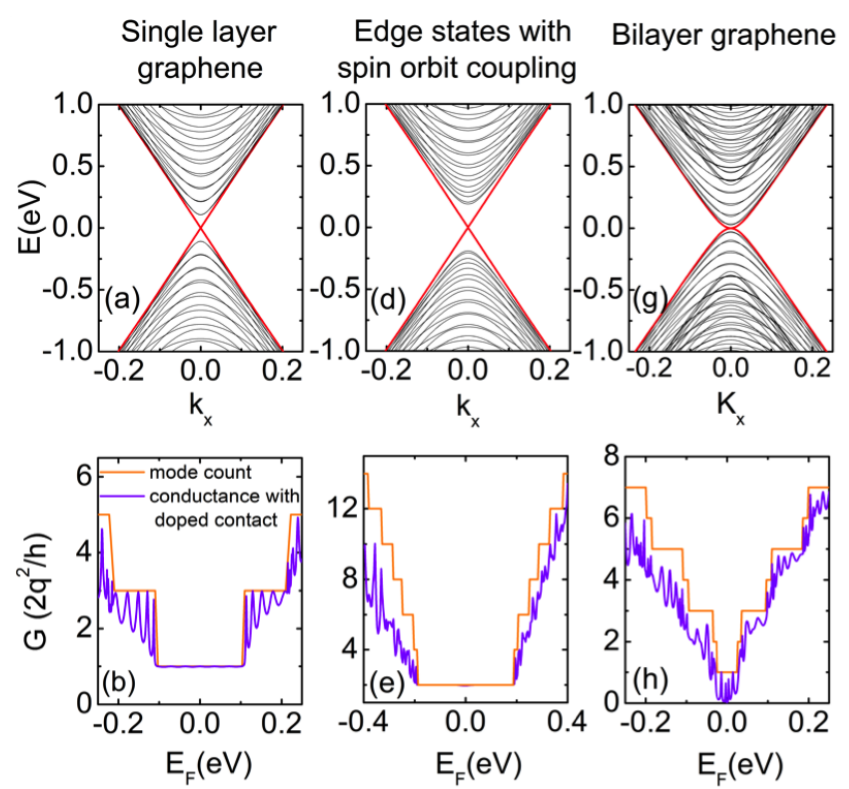

Figure 5.3: Ballistic NEGF calculation of total conductance $G$ of single layer graphene and bilayer graphene reveals electron hole asymmetry and Fabry-Pérot oscillation. The lowest mode in single layer does not show such oscillation but the bilayer does manifesting Klein tunnel (or reflection).

transport behavior near the Dirac point is another incarnation of Klein transmission and reflection in graphene heterojunctions [37].

\subsubsection{Conductance of a double gated structure}

Fig. 5.4 shows transport properties of a double gated graphene device. The middle part of the channel is controlled by both top and bottom gates while the remaining part is controlled by the back gate alone. Fig. 5.4a shows the behavior for non-zero top gate voltage $V_{T G}$ values. A second peak at $\sim-8 \mathrm{~V}$ indicates the shifted Dirac point for the middle part of the channel, which is under top gate. Fig. 5.4c shows the transfer characteristic from experimental measurement [9]. Fig. 5.4c shows the variation of conductance for different lengths for $V_{T G}=0$. For short channels, the boundary potential $V_{B}$ dopes most part of the channel thereby decreasing the peak resistance and shifting its position (experiment in [8], Fig. 5.4d). For diffusive samples, the electron hole asymmetry (EHA) is washed out at larger length due to resistance contribution from the charged impurity scattering, while at 

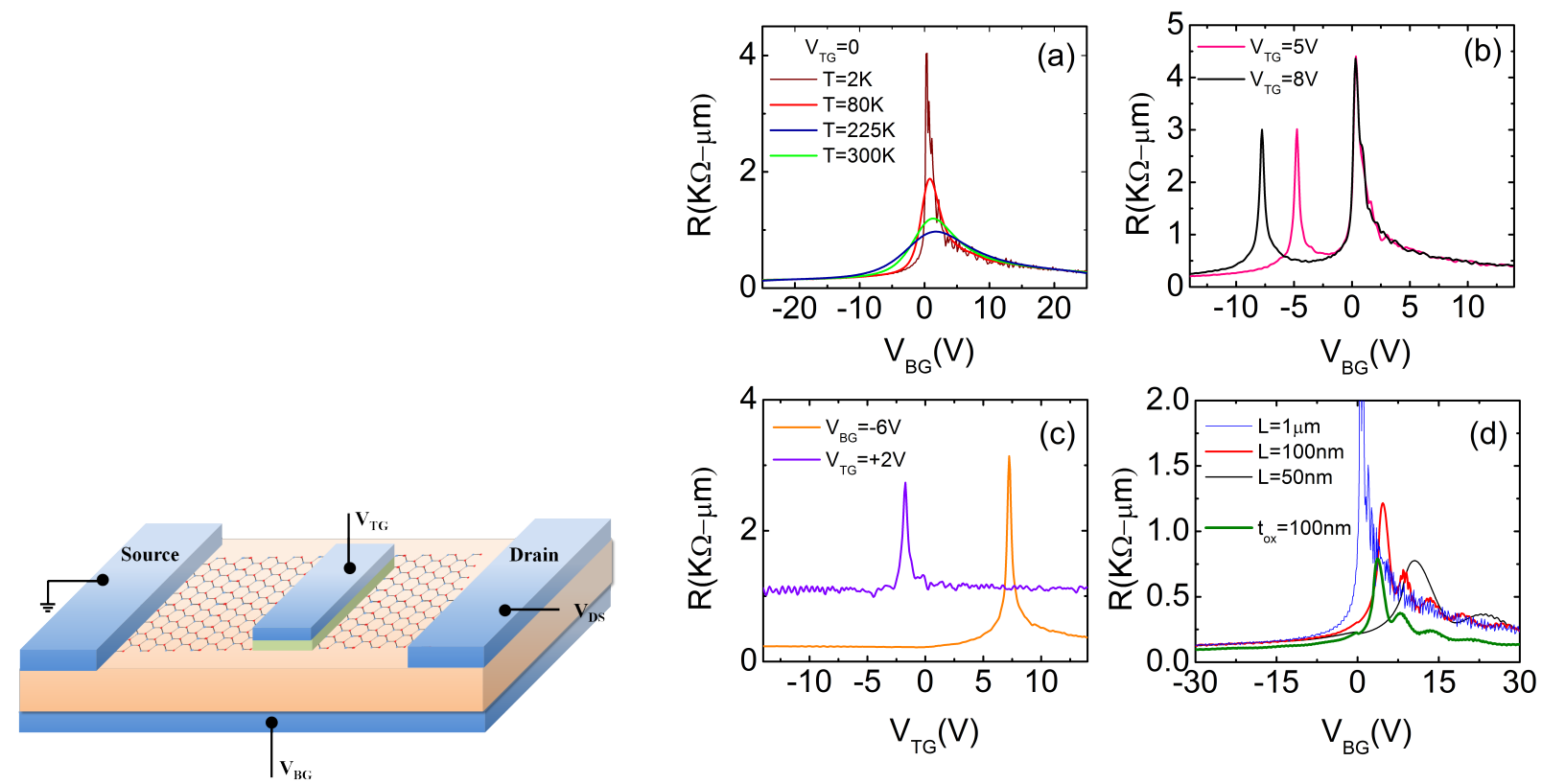

Figure 5.4: Device schematic of a double gate structure (W=1 $\left.\mathrm{Wm}, \mathrm{L}=3 \mu \mathrm{m}, L_{\text {top }}=1 \mu \mathrm{m}\right)$. We show detailed transport behavior, a-b-c) Resistance variation as a function of gate voltages at various temperatures, d) Short channel effects in the device, the boundary potential works as an effective doping and shifts the Dirac point. Decreasing gate oxide thickness increases gate control with peak resistance remaining the same.

shorter lengths the asymmetry along with the shift in peak resistance is recovered. Increasing the gate capacitance on the other hand has the effect of shifting the position of the peak resistance without changing the peak value (Green line in Fig. 5.4c).

\subsubsection{Modeling metal-graphene contact with NEGF}

We briefly revisit the main equations used in our numerical calculations for NEGF. The central quantity calculated in the formalism is the Green's function,

$$
\mathcal{G}(E)=\left[E \mathbb{I}-H-U-\Sigma_{1}-\Sigma_{2}\right]^{-1}
$$

$[H]$ is the tight-binding Hamiltonian matrix of graphene, with a minimal one $p_{z}$ orbital basis per carbon atom. $U$ is the electrostatic potential in the device. $\left[\Sigma_{1,2}\right]$ are the self energy matrices for the semi-infinite source and drain leads calculated from surface Green's 
Chapter 5 | Non-Equilibrium Green's Function (NEGF) formalism: a rigorous transport model66

function $g_{s}$. We calculate $g_{s}$ assuming a doped graphene, with the doping corresponding to the particular metal induced doping (MID, effective doping $\Delta E_{F}$, Fig. 5.5) and thus energy independent,

$$
g_{s}=\left[\Delta E_{F} \mathbb{I}-H-\tau^{\dagger} g_{s} \tau\right]^{-1}
$$

solved iteratively using a decimation technique $[81]$. Then $[\Sigma]=\left[\tau^{\dagger}\right]\left[g_{s}\right][\tau]$, where $[\tau]$ is unit cell to unit cell coupling matrix. The total conductance is calculated from, $G=\operatorname{Tr}\left[\Gamma_{1} \mathcal{G} \Gamma_{2} \mathcal{G}^{\dagger}\right]$ in units of $G_{0}=2 q^{2} / h$, a factor 2 is for spin degeneracy, $\left[\Gamma_{1,2}\right]$ are the anti-Hermitian parts of self energy representing the energy level broadening associated with charge injection and removal in and out of the contact. We adopt a fast partial inversion technique, Recursive Green's Function Algorithm (RGFA) $[82,49]$ version of $[\mathcal{G}]$ to simulate device structures from nano meter up to micron regime. The transmission probability from metal to graphene $T_{M G}$ is assumed to be unity, which is justified for ballistic transport [83]. Therefore, the total conductance at zero temperature from NEGF is equivalent to $G=G_{0} M T_{a v}$, where $M T_{a v}$ accounts for the product of available number of modes $M$ and an average transmission per mode $T_{a v}$ across the heterojunctions [84]. We adopt the MID and boundary potential $V_{B}$ values from Ref. [85], which outlines the nature of the screening potential inside the channel for various metals/metal stacks. The device schematic is shown in Fig. 5.5 along with the potential profile. The channel is controlled by the back gate with oxide thickness $\left(t_{o x}\right) 100 \mathrm{~nm}$. The channel width $(W)$ is set at $1 \mu \mathrm{m}$ and the length $(L) 50 \mathrm{~nm}$. For certain metals (e.g. $\mathrm{Pd} / \mathrm{Au}$ ), one sharp $p n$ junction is always formed at the interface of free standing graphene and the graphene under metal, depending on the relative sign of $\Delta E_{F}$ and $V_{B}$. Due to the asymptotic nature of the decay of boundary potential, another smooth junction is formed inside the channel if the sign of the back gate voltage is different from $V_{B}$. The smooth junction formed within the device contributes to the EHA. The sharp junction on the other hand plays no role in EHA except to increase the contact resistance since it is present 

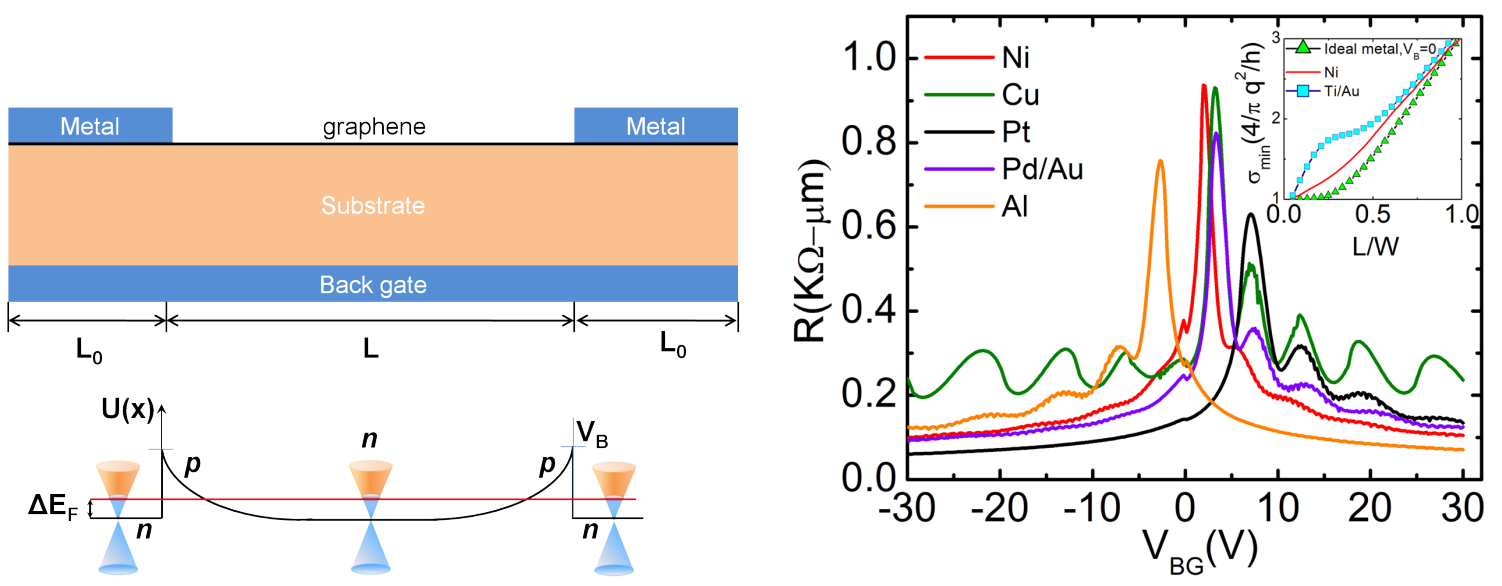

Figure 5.5: Left: device schematic to study metal-graphene interaction. Graphene under metal is doped with $\Delta E_{F}$ and the potential at the boundary (middle) between metal and graphene is $V_{B}$, which decays as $\sim 1 / \sqrt{x / l_{s}}, l_{s}$ being the scaling length. Right: zero temperature transfer characteristics for common metals from NEGF calculation of $50 \mathrm{~nm} \times 1 \mu \mathrm{m}$ graphene, $t_{o x}=100 \mathrm{~nm}$. The boundary potential $V_{B}$ plays the important role of deciding electron hole asymmetry, position and value of the peak resistance while $\Delta E_{F}$ determines the lowest achievable resistance. Inset shows the variation of minimum conductivity $\sigma_{m i n}$, realistic metals show deviation from the ideal picture at short lengths.

regardless of gate voltage. The device potential including both contacts becomes [85]

$$
\begin{aligned}
U(x)= & \operatorname{sign}[\sigma(x)] \hbar v_{f} \sqrt{\frac{\sigma(x)}{q}} \\
\frac{q \cdot \sigma(x)}{\epsilon_{0} \kappa}= & \frac{4}{\pi x}\left(V_{B 2}+V_{B 1} \frac{\pi x / t_{o x}}{e^{\pi x / t_{o x}}-1}\right)-\frac{q \cdot V_{G}}{t_{o x}}+ \\
& \frac{4}{\pi(L-x)}\left(V_{B 2}+V_{B 1} \frac{\pi(L-x) / t_{o x}}{e^{\pi(L-x) / t_{o x}}-1}\right)
\end{aligned}
$$

where $V_{B 1}$ and $V_{B 1}$ are constants based on the work functions. The first and last terms in the definition of carrier density $\sigma$ come from the contacts and the second term is the gate induced carriers. $\kappa \approx 2.5$ is the effective dielectric constant for graphene on $\mathrm{SiO}_{2}$ substrate. Fig. 5.5 shows the transfer characteristics for various metals. We choose five metals, Ni, $\mathrm{Cu}$, $\mathrm{Pt}, \mathrm{Pd} / \mathrm{Au}, \mathrm{Al}$ each of which are fairly distinct from one another. The minimum contact resistance achievable is a function of the MID. For example, $\mathrm{Cu}$ shows the highest resistance [86] $(\sim 300 \Omega-\mu \mathrm{m})$ due its low MID, $\Delta E_{F}=0.08 \mathrm{eV}$. Even though Ni has the highest $\Delta E_{F}$, 
Pt has the lowest resistance $(\sim 80 \Omega-\mu \mathrm{m})$ among all, mainly because of the unipolarity of the sharp junction $\left(p p^{\prime}\right)$ as well as lower potential step at the interface. For non-zero boundary potential $V_{B}$, the shift of the Dirac point, the degree of EHA and the maximum resistance are all determined by $V_{B}$, with $\Delta E_{F}$ playing no role. Pt has the highest $V_{B}=0.51 \mathrm{eV}$, thus showing a shift of $\sim 8 \mathrm{~V}$ of the Dirac point, with Ni showing the lowest shift $\left(V_{B}=0.04\right.$ $\mathrm{eV})$. At the same time, $\mathrm{Pt}$ and $\mathrm{Ni}$ shows the highest and lowest EHA respectively. The peak resistance is lowest for $\mathrm{Pt}$, because of its high $V_{B}$, with $\mathrm{Ni}$ showing the highest peak. The transport is ballistic in the simulation, therefore the total resistance shown in this paper is effectively the 'contact' resistance and should roughly approach the contact resistance extracted from experimental data, commonly by Transfer Length Method (TLM) [83, 87]. The lowest contact resistance is achieved when the channel doping reaches $\Delta E_{F}$, the simulation shows good agreement with experiments in this regard, e.g. for $\mathrm{Pd} / \mathrm{Au}$ stack, it is $~ 110$ $\Omega-\mu \mathrm{m}$, vs. $110 \pm 20 \Omega-\mu \mathrm{m}$ in experiment [83], with roughly 1.5 times increase in resistance in the electron side. All metals considered here except $\mathrm{Al}$ show higher resistance for positive $V_{G}$ because of positive $V_{B}$ (holes) [85], showing increased resistance and higher oscillation in the bipolar regime $\left(V_{G}>0\right)$. Inset in Fig. 5.5 shows the variation of the $\sigma_{\min }$ for a realistic metal, in comparison with the ideal picture as shown in Fig. 5.3. $\sigma_{m i n}$ deviates from $\frac{4 q^{2}}{\pi h}$ especially at short length, where the asymptotic potential effectively dopes the graphene sheet and increases $\sigma_{\min }$.

\subsubsection{Ballistic graphene FET output characteristics}

In this section, we simulate the output characteristics of graphene based field effect transistors. The graphene channel is controlled with a single global back gate. After the self-consistency is achieved between carrier density from NEGF and channel potential from Poisson solver, current is calculated by applying Landauer formalism at room temperature. Fig. 5.6a shows the current calculation for a graphene sheet without the effect of contact induced doping. Since the intrinsic electron hole symmetry, the $I-V$ is symmetric. Lack of bandgap in 

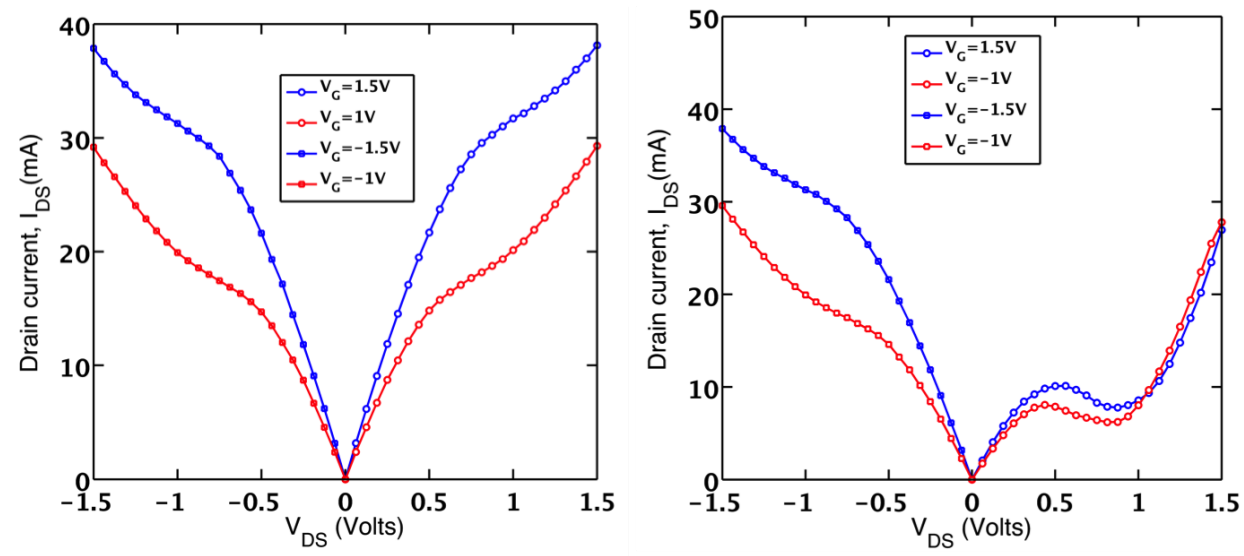

Figure 5.6: Output characteristics of graphene, a) without contact effects showing no saturation due to lack of bandgap, b) with contacts effects (doped contacts) showing negative differential resistance (NDR)
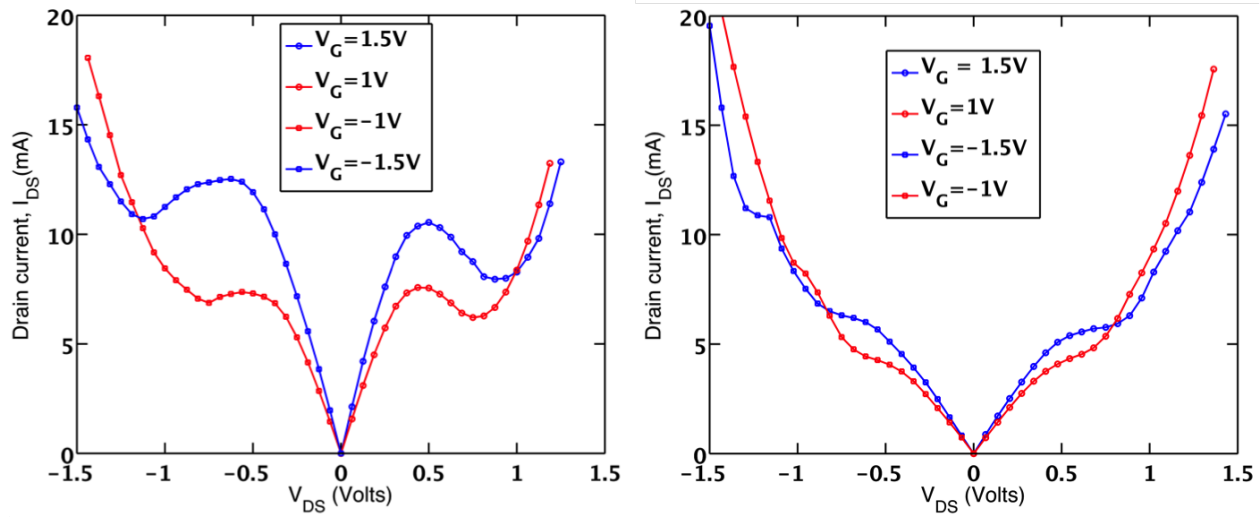

Figure 5.7: Output characteristics of a single graphene pn junction, a) Abrupt junction showing strong NDR, b) Smooth junction with suppressed NDR.

graphene bandstructure leads to no saturation and band to band tunneling. Fig. 5.6b shows the impact of contact induced doping on the $I-V$, which is highly asymmetric with negative MID. For positive $V_{G}$, a bipolar pnp (contact-channel-contact) doping profile is formed (higher resistance) along the channel, compared to unipolar $p p p$ (lower resistance) for negative $V_{G}$. The asymmetry is accompanied with negative differential resistance (NDR) for positive gate voltage, while the negative gate voltage does not produce NDR. The NDR occurs as a result of lower density of states with drain bias in graphene under contact (drain end).

Fig. 5.7 shows the $I-V$ for a single graphene $p n$ junction. Because of the same physics (as contact effects), abrupt GPNJ $I-V$ shows strong NDR. For a smooth GPNJ however, 

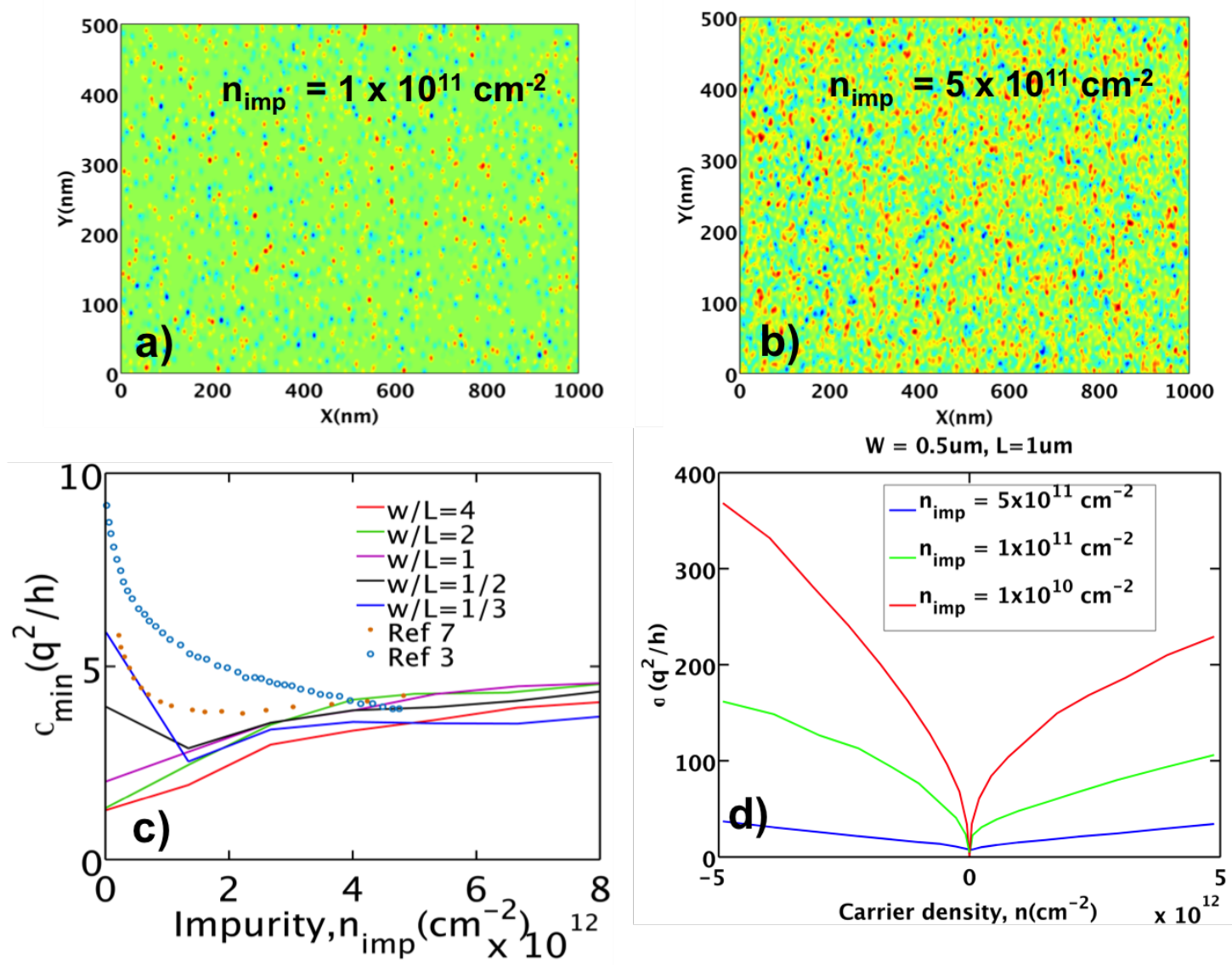

Figure 5.8: Modeling diffusive transport in graphene, a-b) potential landscape for various impurity concentration, c) evolution of minimum conductivity at the Dirac point, d) total conductivity as a function of channel carrier density from ballistic to diffusive. Channel length, $\mathrm{L}$ is $1 \mu \mathrm{m}$ and width, $\mathrm{W}=0.5 \mu \mathrm{m}$

the NDR is suppressed. Such suppression is related to the low conductance of a smooth GPNJ that slows down the increase in current from the beginning.

\subsection{Diffusive transport in graphene}

In this section, we describe how we model diffusive transport of graphene with NEGF. We use a sequence of Gaussian potential profiles for the impurity scattering centers [50], $U(r)=\sum_{n=1}^{N_{i m p}} U_{n} \exp \left(-\left|r-r_{n}\right|^{2} / 2 \zeta^{2}\right)$ specifying the strength of the impurity potential at atomic site $r$, with $r_{n}$ being the positions of the impurity atoms and $\zeta$ the screening length $\left(\approx 3 \mathrm{~nm}\right.$ for long range scatterers). The amplitudes $U_{n}$ are random numbers following a 
Gaussian profile [88], $N_{i m p}$ is the impurity concentration. With $U$ added to $[H]$ (potential landscape shown in Fig. 5.8a-b), we study the evolution of graphene transport from ballistic to diffusive; both near the Dirac point and away from it. Minimum conductivity $\sigma_{\min }$ as a function $N_{i m p}$ is shown in Fig. 5.8c, calculated from an average conductance over hundreds of random impurity configurations. In the ballistic limit, $\sigma_{\min }$ varies linearly with $\mathrm{L} / \mathrm{W}$, but as the sample gets dirtier, the $\sigma_{\min }$ becomes less dependent of L/W. At high impurity limit, $\sigma_{m i n}$ becomes weekly dependent on $N_{i m p}$ and saturates around $3-5 q^{2} / h$. In most experiments, the device length $L$ is larger than width $W$ and therefore see a decreasing trend for $\sigma_{\min }$ vs. $N_{\text {imp }}$ such as Ref. [89]. The evolution of $\sigma_{\min }$ from $\frac{4 q^{2}}{\pi h}$ to $\sim 4 q^{2} / h$ and therefore the missing $\pi$ effect can only be seen for devices with $W>>L$. Fig. 5.8d shows graphene conductivity at various channel carrier density for several impurity concentrations. At ballistic limit, electron-hole asymmetry is due to the formation of $p n$ junction between metal-graphene. At high impurity concentration, metal-graphene contact becomes less dominant and the electron-hole asymmetry vanishes similar to experiments. At the diffusive limit, $\sigma \propto n$ for a sample dominated by long range scatterers and for ballistic limit $\sigma \propto \sqrt{n}$, in consistent with experiments [89].

\subsection{Magnetotransport}

A striking property of graphene is its anomalous integer quantum Hall effect. When the Fermi energy lies between two Landau levels (LL) in presence of a magnetic field, conventional twodimensional electron gases show a vanishing longitudinal resistance $\rho_{x x}=0$ and a quantized Hall conductance $G_{H}=2 q^{2} N / h$ where $N$ is a non-negative integer representing the number of filled Landau levels. In contrast, chiral quasiparticles in graphene exhibiting Berry phase show $[90,91]$ a Hall conductance $G_{H}=\frac{4 q^{2}}{h}(N+1 / 2)$, with LL defined as $E_{n}=\frac{\hbar v_{F}}{r_{C}} \operatorname{sign}(n) \sqrt{2|n|}$, where $r_{C}=\sqrt{\frac{\hbar}{q B}}$ is the cyclotron radius. This non-conventional sequence can be explained with the presence of a LL at $\mathrm{E}=0$ resulting in a four fold degeneracy at zero carrier density 
(a)
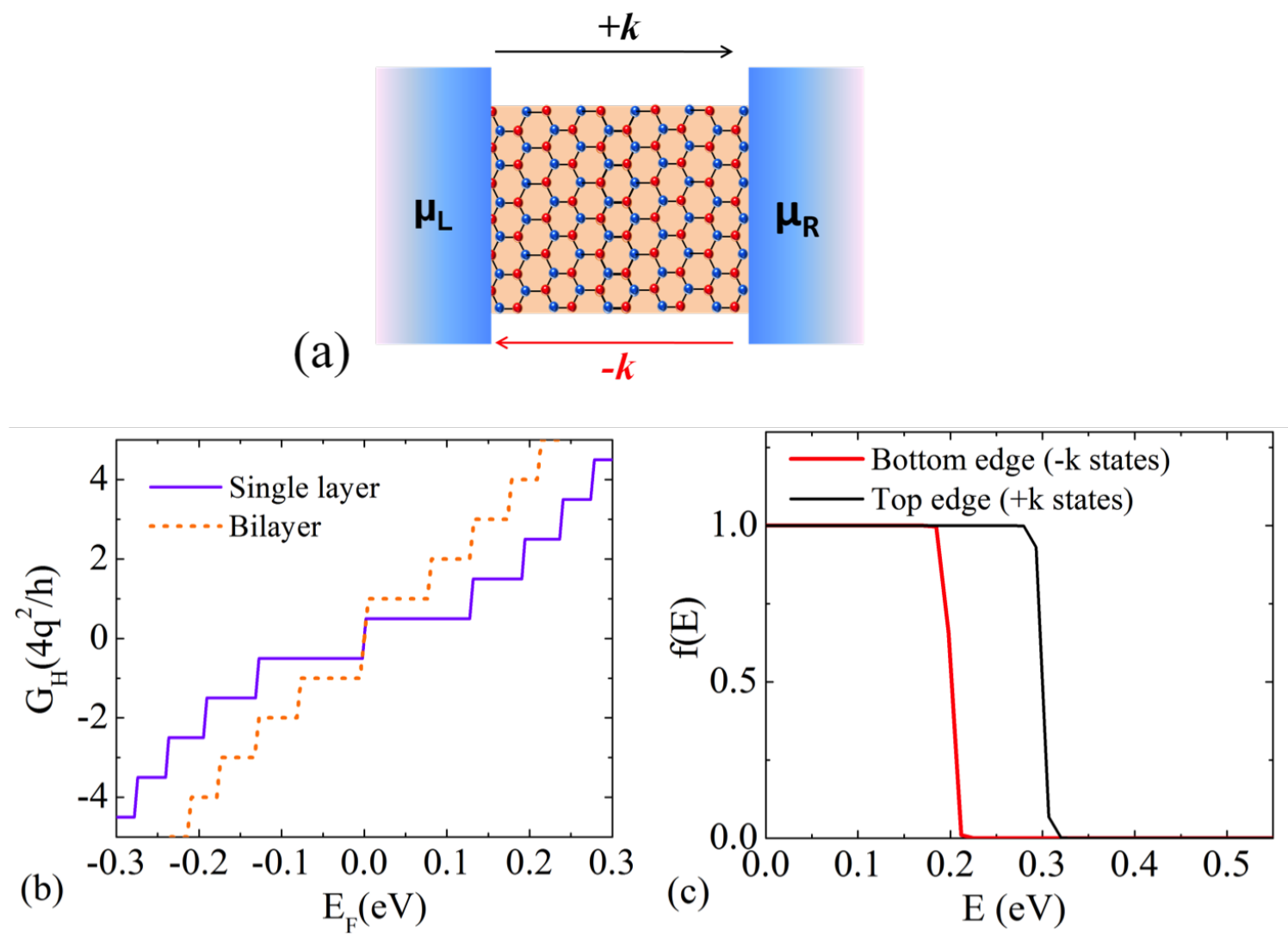

Figure 5.9: (a) In presence of a magnetic field, the current is carried by the edge states that separate into $+k$ states at the upper edge in equilibrium with the left contact and $-k$ states at the lower edge in equilibrium with the right contact. (b) The Hall voltage goes through plateaus at $\frac{4 q^{2}}{h}(N+1 / 2)$ for single layer and $\frac{4 q^{2}}{h}(N+1)$ for bilayer graphene with $N$ a non-negative integer. Note the presence of a jump at zero energy, which is not seen in a two-dimensional free electron gas (and arises from a half-filled Landau level at the Dirac point), and the additional factor of 2 in the plateau heights, arising from valley degeneracy. (c) For a placement of the Fermi energy between two Landau levels, electron distribution function $\mathrm{f}(\mathrm{E})$ at the top and bottom edges resemble Fermi-Dirac distributions of the corresponding contacts. Since each current carrying state sees a constant electrochemical potential (along transport direction $x$ ), the longitudinal resistance vanishes.

(from spin and valley degeneracy). On the contrary, Bilayer graphene LLs are defined as, $E_{n}=\hbar \omega_{C} \sqrt{n(n-1)}$, where $\omega_{C}$ is the cyclotron frequency. Now both $E_{0}$ and $E_{1}$ are at zero energy producing thereby an overall eightfold degeneracy at zero carrier density and QHE plateaus of $G_{H}=\frac{4 q^{2}}{h}(N+1)[92]$.

Numerical modeling of magnetotransport in graphene requires a minor modification to the transport scheme outlined earlier. We replace the kinematic momentum with the 
quasi-momentum $\vec{k} \rightarrow \vec{k}-q \vec{A} / h$, so that the plane wave terms in the Bloch representation pick up an additional phase of $e^{-i q / h \int \vec{A} \cdot \overrightarrow{d l}}$ where $A$ is the magnetic vector potential such that $\vec{\nabla} \times \vec{A}=\vec{B}$. Thus the hopping parameters between atoms 'm' and 'n' are now given by

$$
t_{m n}=t_{0} \exp \left[i \frac{q}{h} \int_{m}^{n} \vec{A} \cdot \overrightarrow{d l}\right]
$$

For $z$ directed magnetic field, our guage is, $A=(-B y, 0,0)$. We can now turn on a magnetic field perpendicular to the sheet, modify the hopping terms as above, and extract the transverse Hall conductance as a function of varying Fermi energy location. The Hall conductance $G_{H}=I / V_{H}$, where $I=(2 q / h) N\left(\mu_{L}-\mu_{R}\right)$ is the current, $N$ is the number of filled Landau levels and the Hall voltage $V_{H}=q \times$ [the difference between the electrochemical potentials of the $+k$ and $-k$ edge states]. These states represent skipping orbits along the edges created by the cyclotron orbits in the bulk, and are separately in equilibrium with the left and right contact Fermi energies Fig. 5.9a.

Fig. 5.9b shows the calculated Hall conductance yielding a series of plateaus for both single layer and bilayer graphene. Fig. 5.9c shows the local carrier distribution functions $f( \pm \vec{k})$ obtained from the ratio of the local carrier density and the local density of states, in other words, the ratio

$$
f(E)=\mathcal{G}_{L, L}^{n}(l, l) / i\left(\mathcal{G}_{L, L}(l, l)-\mathcal{G}_{L, L}(l, l)^{\dagger}\right)
$$

where $l$ is the index for an atom belonging to either top or bottom edge. The Hall voltage turns out to be $V_{H}=q\left(\mu_{L}-\mu_{R}\right)$ (Fig. 5.9c) giving conductance plateaus for $G_{H}$. The electrochemical potentials $\left(\mu_{L}, \mu_{R}\right)$ don't change along transport direction $x$ so that the longitudinal resistance (given by the drop in electrochemical potential along the channel) is zero. 
Chapter 5 | Non-Equilibrium Green's Function (NEGF) formalism: a rigorous transport model74

\subsection{Spin transport}

Given the density matrix, $G^{n}=2 \pi\{\Psi\}\{\Psi\}^{\dagger}$, we can calculate the expected value of an observable $\hat{O}$,

$$
\langle\hat{O}\rangle=\frac{1}{2 \pi} \operatorname{Tr}\left[\hat{O} G^{n}\right]
$$

According to the above equation, the total spin angular momentum and the rate of change of it can be written as,

$$
\begin{aligned}
\langle\vec{S}\rangle & =\frac{\hbar}{4 \pi} \operatorname{Tr}\left\{\tilde{\sigma} \mathrm{G}^{\mathrm{n}}\right\} \\
\frac{\partial\langle\vec{S}\rangle}{\partial t} & =\frac{1}{2 \pi} \operatorname{Tr}\left\{\tilde{\sigma} \mathrm{I}_{\mathrm{op}}\right\}
\end{aligned}
$$

where $\vec{S}=\frac{\hbar}{2} \vec{\sigma} . I_{o p}$ is the charge current operator. From the basic NEGF equation, it can be shown that,

$$
I_{o p}=\frac{i}{2 \pi \hbar}\left[G^{n} H-H G^{n}+G^{n} \Sigma^{\dagger}-\Sigma G^{n}+G \Sigma^{i n}-\Sigma^{i n} G^{\dagger}\right]
$$

And the terminal spin current,

$$
I_{m \vec{s}}=\frac{i}{4 \pi} \operatorname{Tr}\left\{\vec{\sigma}\left[G^{n} \Sigma_{m}^{\dagger}-\Sigma_{m} G^{n}+G \Sigma_{m}^{i n}-\Sigma_{m}^{i n} G^{\dagger}\right]\right\}
$$

This allows us to calculate spin current using NEGF.

\section{Related publications:}

1. Redwan N. Sajjad, Carlos Polanco, Avik Ghosh, "Atomistic deconstruction of current flow in graphene based heterojunctions", Journal of Computational Electronics, invited vol. 12, no. 2, pp. 232 (2013). 


\section{Chapter 6}

\section{Conclusion}

The aim of the research is to design and quantify a novel switching mechanism with graphene, based on the physics of angle dependent tunneling in $p n$ junction. We have demonstrated the concept of transmission gap instead of bandgap in order to get high ON-OFF ratio in graphene. The transmission gap is achieved by filtering out all propagating modes by multiple junctions oriented in such a way so to selectively suppress different modes based on their angles. Such approach retains the intrinsic mobility of graphene intact and decreases the OFF state current without compromising the ON state current. We have shown that the transmission gap results from geometry dependent transport in graphene $p n$ junction. We have worked out a detailed theoretical model of gate geometry dependent transport in GPNJ, that forms the basis of the device designs. Our understanding of the GPNJ transport is complemented by our modeling of recent experiments on similar devices. We have also shown that the transmission gap is gate voltage dependent, it exists only in bipolar $p n$ regime and collapses in the uniform $n n$ (or $p p$ ) limit. Such voltage dependent transmission gap yields steep subthreshold slope in the transfer characteristic of the devices, since the gap changes with the applied gate voltage. This constitutes a new method of beating thermal switching limit $(K T \ln (10) / q)$. We have shown that combining the high mobility of graphene with the steep subthreshold slope, GPNJ based logic circuits can be energy efficient compared to 
Silicon CMOS based logic. Future work in this area can focus on the challenges of GPNJ based logic implementation in presence of non-idealities such as charged impurity scattering, phonon scattering and poor contact resistance. It is also worth investigating the scope of Binary Decision Diagram (BDD) and reconfigurable logic using GPNJ, especially using multiple contacts and using GPNJ's ability to deflect electrons at particular angles. We identified that edge reflection is the main challenge to overcome for the device idea, therefore device deigns that are immune to edge reflections will be quite attractive.

We have also presented quantum transport calculation of graphene devices using NEGF simulation. We have benchmarked a number of experiments in both ballistic and diffusive regime. Future work in this regard may include a comprehensive phonon scattering model in graphene using NEGF. Also, studies on the effect of self-heating at high bias and the role of various substrates are lacking in the literature. In addition, the competition between phonon scattering, impurity scattering and self-heating on the current saturation is not well understood from a bottom up quantum transport model and can be the topic of future work.

Topological Insulator (TI) is considered to be attractive for spintronic devices because of its intrinsic spin polarized current on its surface. We have shown how the chiral properties of TI can be used with a single pn junction to increase spin current and reduce charge current at the same time, in much the same way as graphene. A pn junction in graphene like systems where the electronic structure is described by a Dirac type Hamiltonian with chiral properties, only allows low angle electrons (up pseudospin) to go through. We have demonstrated that such filtering takes place in TI, originating from the similar bandstructure on its surface, but this time the filtering occurs on real spins, instead of pseudospins. Using a smooth junction with practical transition length and width, we have shown that the spin to charge current ratio can be as high as 20 , almost two orders of magnitude higher than giant spin Hall systems. Spin polarized current accompanied by low charge current has important application in spin based logic and memory devices. Although the magnitude of spin current on the surface of TI is smaller compared to a 3D system, TI can still be appealing because 
we have demonstrated a gate controllable spin current. Future research may pursue possible logic and memory schemes based on the device we proposed. 


\section{Appendix A}

\section{GPNJ transmission equations}

In this appendix, we derive the transmission probability equations in GPNJ.

\section{A.1 Abrupt step potential (abrupt GPNJ)}

Graphene's wave-functions are composed of a two-component spinor $\left(\begin{array}{lll}\psi_{1} & \psi_{2}\end{array}\right)$ signifying the contribution from $A$ and $B$ sub-lattices. We can write

$$
\begin{gathered}
\psi_{1}= \begin{cases}\left(e^{i k_{x} x}+r e^{-i k_{x} x}\right) e^{i k_{y} y} & x<0 \\
t e^{i q_{x} x+i k_{y} y} & x>0\end{cases} \\
\psi_{2}= \begin{cases}s_{1}\left(e^{i k_{x} x+i \theta_{1}}-r e^{-i k_{x} x-i \theta_{1}}\right) e^{i k_{y} y} & x<0 \\
s_{2} t e^{i q_{x} x+i k_{y} y+i \theta_{2}} & x>0\end{cases}
\end{gathered}
$$

The transverse momentum $k_{y}$ is conserved across the junction,

$$
k_{F i} \sin \theta_{i}=k_{F i} \sin \theta_{r}=k_{F t} \sin \theta_{t}
$$

In case of GPNJ, the transmitted wavevector's $x$ component must change sign to keep the 
velocity in the same direction. $q_{x}=-k_{x}$ and $\theta_{t}=-\theta_{i}$ for symmetric GPNJ. Therefore, $\sin \theta_{i} / \sin \theta_{t}=-k_{F t} / k_{F i}=-n$. This is Snell's law for Graphene with negative refractive index ratio. For asymmetric junction, $\left|q_{x}\right|=\left|k_{x}\right| \sqrt{\sin ^{2} \theta_{C}-\tan ^{2} \theta_{i} \cos ^{2} \theta_{C}}$. Now let us calculate the transmission and reflection co-efficients by matching the boundary conditions

$$
\begin{aligned}
& \psi=\psi_{i}+\psi_{r} ; \quad x<0 \\
& \psi=\psi_{t} ; \quad x>0
\end{aligned}
$$

for $\mathrm{x}=\mathrm{y}=0$, from the first and second row (Eq. A.1-A.2)

$$
\begin{aligned}
1+r & =t \\
e^{i \theta_{i}}-r e^{-i \theta_{i}} & =t e^{i \theta_{t}}
\end{aligned}
$$

solving the two equations for $r$ and $t$ gives

$$
\begin{aligned}
t & =\frac{2 \cos \theta_{i}}{e^{-i \theta_{i}}+e^{i \theta_{t}}} \Rightarrow|t|^{2}=\frac{\cos ^{2} \theta_{i}}{\cos ^{2}\left(\frac{\theta_{i}+\theta_{t}}{2}\right)} \\
r & =\frac{e^{i \theta_{i}}-e^{i \theta_{t}}}{e^{-i \theta_{i}}+e^{i \theta_{t}}} \Rightarrow|r|^{2}=\frac{1-\cos \left(\theta_{i}-\theta_{t}\right)}{1+\cos \left(\theta_{i}+\theta_{t}\right)}
\end{aligned}
$$

And the reflection and transmission probabilities

$$
\begin{aligned}
R & =|r|^{2}=\frac{1-\cos \left(\theta_{i}-\theta_{t}\right)}{1+\cos \left(\theta_{i}+\theta_{t}\right)} \\
T & =\frac{v_{x t}}{v_{x i}}|t|^{2}=\frac{v_{F} \cos \theta_{t}}{v_{F} \cos \theta_{i}}|t|^{2}=\frac{\cos \theta_{i} \cos \theta_{t}}{\cos ^{2}\left(\frac{\theta_{i}+\theta_{t}}{2}\right)}
\end{aligned}
$$

for $\theta_{i}<\theta_{C}$ and 0 otherwise. It can be shown that $1=R+T$, as expected. The transmission probability can be written as

$$
T\left(\theta_{i}, E, V_{G}\right)=\Theta\left(\theta_{c}-\theta_{i}\right) \frac{\cos \theta_{i} \cos \theta_{t}}{\cos ^{2}\left(\frac{\theta_{i}+\theta_{t}}{2}\right)}
$$


where $\Theta$ is the unit step function. The energy dependence of T comes through $\theta_{t}$ and $\theta_{C}$. From Snell's law -

$$
\begin{aligned}
\theta_{t} & =\sin ^{-1}\left(\frac{k_{F i}}{k_{F t}} \times \sin \theta_{i}\right)=\sin ^{-1}\left(\frac{\Delta E_{1}}{\Delta E_{2}} \times \sin \theta_{i}\right) \\
\theta_{C} & =\sin ^{-1}\left(\frac{k_{F t}}{k_{F i}}\right)=\sin ^{-1}\left(\frac{\Delta E_{1}}{\Delta E_{2}}\right)
\end{aligned}
$$

Here, $\Delta E_{1,2}$ are doping levels on both sides set by the gate voltages $V_{G 1}$ and $V_{G 1}$.

Now let us analyze the transmission equation for some specific cases

Case 1: Same material $\theta_{i}=\theta_{t}, \mathrm{~T}(\theta)=1$

Case 2: Focus $\theta_{i}=-\theta_{t}, \mathrm{~T}(\theta)=\cos ^{2} \theta_{i}$

Case 3: Total internal reflection $\theta_{i}=\theta_{C}, \theta_{t}=90^{\circ}, \mathrm{T}(\theta)=0$. Equations A.9 come from continuity equation for the Dirac Hamiltonian.

\section{Transmission from continuity equation of Graphene}

$$
i \hbar \frac{\partial \psi}{\partial t}=H \psi
$$

Hamiltonian for graphene

$$
\begin{gathered}
H=v_{F} \vec{\sigma} \cdot \vec{p} \\
i \hbar \frac{\partial}{\partial t}\left(\begin{array}{c}
\psi_{1} \\
\psi_{2}
\end{array}\right)=\hbar v_{F}\left(\begin{array}{cc}
0 & k_{x}-i k_{y} \\
k_{x}+i k_{y} & 0
\end{array}\right)\left(\begin{array}{l}
\psi_{1} \\
\psi_{2}
\end{array}\right)
\end{gathered}
$$


Now using $k_{x}=-i \frac{\partial}{\partial x}$ and $k_{y}=-i \frac{\partial}{\partial y}$ we get

$$
\begin{aligned}
& \frac{\partial \psi_{1}}{\partial t}=-v_{F}\left(\frac{\partial}{\partial x}-i \frac{\partial}{\partial y}\right) \psi_{2} \\
& \frac{\partial \psi_{2}}{\partial t}=-v_{F}\left(\frac{\partial}{\partial x}+i \frac{\partial}{\partial y}\right) \psi_{1}
\end{aligned}
$$

And carrier density

$$
\begin{aligned}
\rho & =\psi^{*} \psi=\left(\begin{array}{ll}
\psi_{1}^{*} & \psi_{2}^{*}
\end{array}\right)\left(\begin{array}{c}
\psi_{1} \\
\psi_{2}
\end{array}\right) \\
\rho & =\psi_{1}^{*} \psi_{1}+\psi_{2}^{*} \psi_{2} \\
\frac{\partial \rho}{\partial t} & =\psi_{1}^{*} \frac{\partial \psi_{1}}{\partial t}+\frac{\partial \psi_{1}^{*}}{\partial t} \psi_{1}+\psi_{2}^{*} \frac{\partial \psi_{2}}{\partial t}+\frac{\partial \psi_{2}^{*}}{\partial t} \psi_{2} \\
\frac{\partial \rho}{\partial t} & =-v_{F}\left[\frac{\partial}{\partial x}\left(\psi_{1}^{*} \psi_{2}+\psi_{1} \psi_{2}^{*}\right)+i \frac{\partial}{\partial y}\left(\psi_{1} \psi_{2}^{*}-\psi_{1}^{*} \psi_{2}\right)\right] \\
\frac{\partial \rho}{\partial t} & ==-\left(\frac{\partial J_{x}}{\partial x}+\frac{\partial J_{y}}{\partial y}\right)=-\vec{\nabla} \cdot \vec{J}
\end{aligned}
$$

where current density $J$ can be defined as

$$
\begin{gathered}
J_{x}=v_{F}\left(\psi_{1}^{*} \psi_{2}+\psi_{1} \psi_{2}^{*}\right)=v_{F} \psi^{*} \sigma_{x} \psi \\
J_{y}=i v_{F}\left(\psi_{1} \psi_{2}^{*}-\psi_{1}^{*} \psi_{2}\right)=v_{F} \psi^{*} \sigma_{y} \psi
\end{gathered}
$$

enabling us to write,

$$
J=v_{F} \psi^{*} \sigma \psi
$$


We consider the two component wavevector of graphene,

$$
\begin{aligned}
& \psi_{i}=\left(\begin{array}{c}
1 \\
e^{i \theta_{i}}
\end{array}\right) e^{i \overrightarrow{k_{i} \cdot \vec{r}}} \\
& \psi_{r}=r\left(\begin{array}{c}
1 \\
-e^{-i \theta_{i}}
\end{array}\right) e^{i \overrightarrow{k_{r} \cdot \vec{r}}} \\
& \psi_{t}=t\left(\begin{array}{c}
1 \\
e^{i \theta_{t}}
\end{array}\right) e^{i \overrightarrow{k_{t} \cdot \vec{r}}}
\end{aligned}
$$

Taking only the $x$ components of the current density

$$
\begin{aligned}
J_{i}=v_{F}\left(\psi_{1 i}^{*} \psi_{2 i}+\psi_{1 i} \psi_{2 i}^{*}\right) & =v_{F}\left(e^{i \theta_{i}}+e^{-i \theta_{i}}\right) \\
J_{r}=v_{F}\left(\psi_{1 r}^{*} \psi_{2 r}+\psi_{1 r} \psi_{2 r}^{*}\right) & =-v_{F}|r|^{2}\left(e^{i \theta_{i}}+e^{-i \theta_{i}}\right) \\
J_{t}=v_{F}\left(\psi_{1 t}^{*} \psi_{2 t}+\psi_{1 t} \psi_{2 t}^{*}\right) & =v_{F}|t|^{2}\left(e^{i \theta_{t}}+e^{-i \theta_{t}}\right)
\end{aligned}
$$

And transmission and reflection co-efficients,

$$
\begin{array}{r}
R=\frac{\left|J_{r}\right|}{\left|J_{i}\right|}=|r|^{2} \\
T=\frac{\left|J_{t}\right|}{\left|J_{i}\right|}=|t|^{2} \frac{e^{i \theta_{t}}+e^{-i \theta_{t}}}{e^{i \theta_{i}}+e^{-i \theta_{i}}} \\
T=\frac{v_{F} \cos \theta_{t}}{v_{F} \cos \theta_{i}}|t|^{2}=\frac{v_{x t}}{v_{x i}}|t|^{2}
\end{array}
$$

\section{General expression}

We can derive a general expression that will work even if the incidence angle is more than 
the critical angle.

$$
\begin{aligned}
r & =\frac{e^{i \theta_{i}}-e^{i \theta_{2}}}{e^{-i \theta_{i}}+e^{i \theta_{2}}} \\
r^{*} & =\frac{e^{-i \theta_{i}}-e^{-i \theta_{2}^{*}}}{e^{i \theta_{i}}+e^{-i \theta_{2}^{*}}} \\
R=|r|^{2}=r . r^{*} & =\frac{\left(e^{i \theta_{i}}-e^{i \theta_{2}}\right)}{\left(e^{-i \theta_{i}}+e^{i \theta_{2}}\right)} \frac{\left(e^{-i \theta_{i}}-e^{-i \theta_{2}^{*}}\right)}{\left(e^{i \theta_{i}}+e^{-i \theta_{2}^{*}}\right)}
\end{aligned}
$$

Thus,

$$
\begin{aligned}
R & =\frac{1-e^{i\left(\theta_{2}-\theta_{1}\right)}-e^{-i\left(-\theta_{1}^{*}+\theta_{2}^{*}\right)}+e^{i\left(\theta_{2}-\theta_{2}^{*}\right)}}{1+e^{i\left(\theta_{2}+\theta_{1}\right)}+e^{-i\left(\theta_{1}^{*}+\theta_{2}^{*}\right)}+e^{i\left(\theta_{2}-\theta_{2}^{*}\right)}} \\
R & =\frac{1-e^{-2 \theta_{2 I}}\left[2 \cos \left(\theta_{2 R}-\theta_{1}\right)-e^{\left.-\theta_{2 I}\right]}\right.}{1+e^{-2 \theta_{2 I}\left[2 \cos \left(\theta_{2 R}+\theta_{1}\right)+e^{-\theta_{2 I}}\right]}}
\end{aligned}
$$

And

$$
T=\frac{4 e^{-2 \theta_{2 I}} \cos \theta_{2 R} \cos \theta_{1}}{1+e^{-2 \theta_{2 I}\left[2 \cos \left(\theta_{2 R}+\theta_{1}\right)+e^{-\theta_{2 I}}\right]}}
$$

When the incident angle $\theta_{i}>\theta_{C}$, then the expression of the refracted angle becomes

$$
\theta_{t}=\alpha+i \beta=\pi / 2+i \cosh ^{-1}(A)
$$

Putting this back to Eq. A.23

$$
R=\frac{1+e^{-2 \theta_{2 I}}-2 e^{-\theta_{2 I}} \sin \theta_{1}}{1+e^{-2 \theta_{2 I}}-2 e^{-\theta_{2 I}} \sin \theta_{1}}=1
$$

For cases $\theta_{1}<\theta_{C}, \theta_{2 I}=0$ and we have

$$
R=\frac{1-\cos \left(\theta_{2}-\theta_{1}\right)}{1+\cos \left(\theta_{2}+\theta_{1}\right)}
$$

and $T$ reduces to what we derived earlier. 


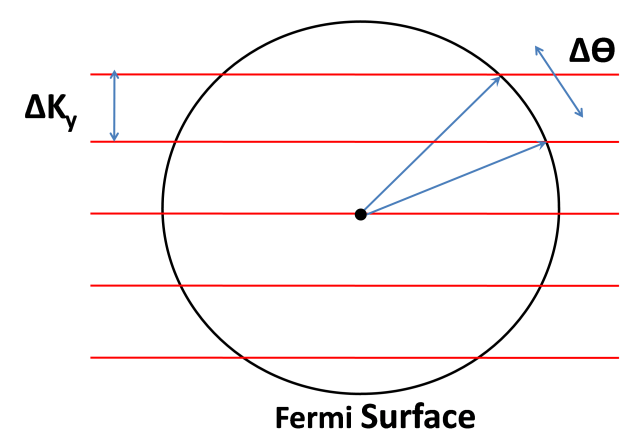

Figure A.1: Transverse modes indicated by red horizontal lines

\section{Conductance calculation}

Conductance at Fermi Energy $E_{F}$ for a graphene sheet according to Landauer formula,

$$
G=\frac{2 q^{2}}{h} M\left(E_{F}\right) T_{a v}
$$

where $M\left(E_{F}\right)$ is the number of modes and $\theta=\tan ^{-1}\left(\frac{k_{y}}{k_{x}}\right)$ is determined by individual modes. For uniformly gated graphene, $T_{a v}$ is unity for ballistic transport. The number of modes is given by

$$
\begin{gathered}
M\left(E_{F}\right)=\frac{2 W|E|}{\pi \hbar v_{F}} \\
G=\frac{4 q^{2}}{\pi h} k_{F} W
\end{gathered}
$$

following $E_{F}=\hbar v_{F} k_{F}$. For a symmetric $p n$ junction

$$
T(\theta)=\cos ^{2}(\theta)
$$

The conductance becomes, 


$$
\begin{aligned}
G\left(E_{F}\right) & =G_{0} \sum T(\theta)=G_{0} \int \frac{T(\theta)}{\Delta \theta} d \theta \\
& =G_{0} \frac{k_{F}}{\Delta k_{y}} \int T(\theta) \cos \theta d \theta \quad\left[\text { Using } \Delta k_{y}=k_{F} \cos \theta \Delta \theta \text { from Fig. A.1 }\right] \\
& =G_{0} M\left(E_{F}\right) \frac{1}{2} \int \cos ^{2} \theta \cos \theta d \theta \\
& =\frac{2}{3} G_{0} M\left(E_{F}\right)
\end{aligned}
$$

$2 / 3 \mathrm{rd}$ of uniformly doped graphene.

\section{A.2 Split gated structure: smooth variation of poten- tial and collimation}

For a smoothly varying potential, the transverse momentum $k_{y}$ is still conserved across the junction. Therefore the longitudinal wavevector $k_{x}$ becomes evanscent due to the reduction of doping over a region $a<x<b$ defined by

$$
\begin{aligned}
& E-U(a)=\hbar v_{F} k_{y} \\
& E-U(b)=-\hbar v_{F} k_{y}
\end{aligned}
$$

And the transmission is calculated from WKB approximation [43]

$$
\begin{aligned}
T(E, \theta) & =e^{-2 S} \\
S & =\int_{a}^{b} \kappa(x) d x \\
& =\int_{a}^{b} \sqrt{k_{y}^{2}-\left(\frac{E-U_{0} x /(2 d)}{\hbar v_{F}}\right)^{2}} d x
\end{aligned}
$$




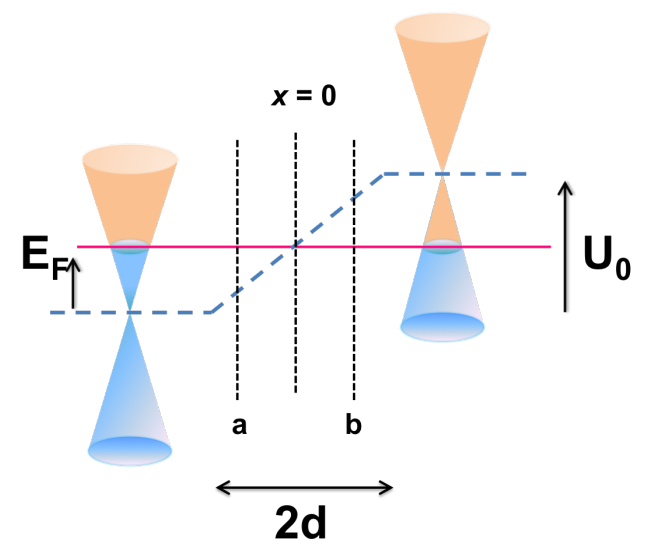

Figure A.2: Energy band diagram of a smooth $p n$ junction with the forbidden region

After performing the integration, we get

$$
T(E, \theta)=e^{-\pi \frac{d_{e f f}}{2} k_{F 1}|\sin \theta|}
$$

where the effective barrier width is defined as,

$$
d_{e f f}=b-a=\frac{2 \hbar v_{F}\left|k_{y}\right|}{U_{0}}(2 d)
$$

\section{Conductance calculation:}

For a smooth pn junction

$$
T(\theta)=e^{-\pi k_{F} d \sin ^{2}(\theta)}
$$

This gives

$$
\begin{aligned}
G & =\frac{4 q^{2}}{h} \int \frac{1}{\Delta \theta} e^{-k_{F} d \sin ^{2} \theta} d \theta \\
G & =\frac{4 q^{2}}{h} \frac{k_{F} W}{2 \pi} \int e^{-k_{F} d \sin ^{2} \theta} d \theta
\end{aligned}
$$


It selectively transmits modes only within $\theta_{0} \leq\left(\pi k_{F} d\right)^{-1 / 2}$ [43]. The conductance becomes

$$
\begin{aligned}
G & \approx \frac{4 q^{2}}{h} \frac{k_{F} W}{\pi} \frac{1}{2} \frac{\sqrt{\pi}}{\sqrt{\pi k_{F} d}} \\
G & =\frac{4 q^{2}}{\pi h} \frac{1}{2} \sqrt{\frac{k_{F}}{d}} W
\end{aligned}
$$

assuming $k_{F} d>>1$ and angles $\theta$ not too close to $\pi / 2$. The conductance is lower than uniformly doped graphene by a factor of factor of $2 \sqrt{k_{F} d}$ (compare with Eq. A.31).

General expression of $T_{a v}$ vs. $E$ for a smooth junction We know, $k_{F 1}=E / \hbar v_{F}$, $k_{F 2}=\left(U_{0}-E\right) / \hbar v_{F}$ so, $U_{0}=\hbar v_{F}\left(k_{F 1}+k_{F 2}\right)$, putting this back to Eq. A.35 gives,

$$
T(E, \theta)=e^{\frac{-\sqrt{\pi} k_{F 1}^{2} 2 d \sin ^{2} \theta}{\sqrt{n} 1+\sqrt{n}} 2}
$$

The critical angle condition comes into play through the effective barrier width, which becomes infinitely wide if,

$$
\begin{array}{r}
b<d_{2} \\
\frac{E+\hbar v_{F} k_{y}}{U_{0}} d<\frac{U_{2}}{U_{0}} d \\
\sin \theta_{1}<\frac{E-U_{2}}{E-U_{1}}
\end{array}
$$

which defined critical angle. $U_{1,2}$ are the Dirac points on both sides and $d_{1,2}$ are the positions of the Dirac points. Combining the two formulae, and taking the average over all modes, we get Fig. A.3 which matches closely with the numerical calculation presented in Chapter 2. Average transmission per mode as a function of energy,

$$
T_{a v}(E)=\frac{1}{2} \int_{-\pi / 2}^{\pi / 2} d \theta f(\theta) e^{-\pi d\left(\frac{E-U_{1}}{U_{0}}\right) k_{F 1} \sin ^{2} \theta}
$$




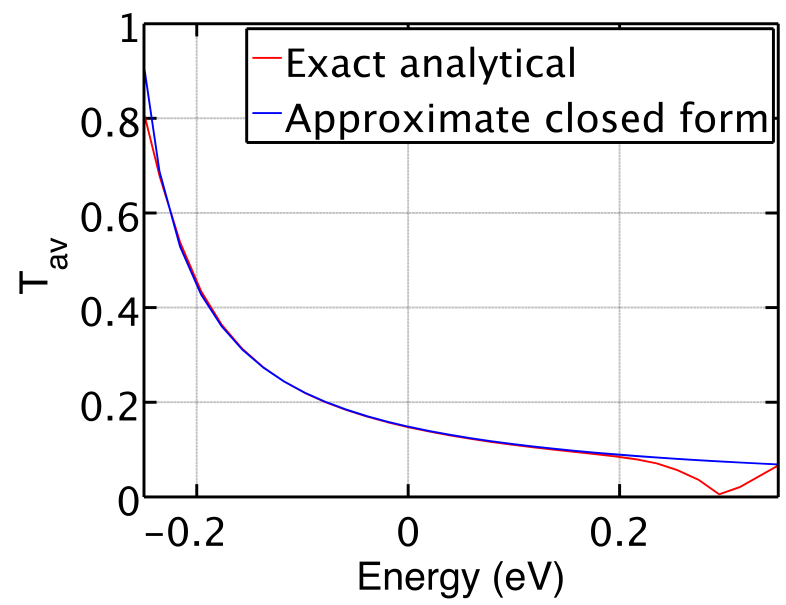

Figure A.3: Exact analytical vs. approximate closed form expression, Eq. A.47

where $f(\theta)$ is the wavefunction mismatch we derived earlier. Within the barrier, where transmission is dominated by tunneling, we can write

$$
T_{a v}(E) \approx \frac{1}{2}\left(\frac{1}{E-U_{1}}\right) \sqrt{\frac{U_{0} \hbar v_{F}}{2 d}}
$$

for energy not too close to the Dirac points. Outside the barrier, the transmission depends on the wavefunction mismatch only. 


\section{Appendix B}

\section{Transmission gap in multiple $p n$ junction device}

The average transmission per mode

Total transmission through a graphene heterojunction can be written as,

$$
\begin{aligned}
G\left(E_{F}\right) & =G_{0} \sum T(\theta)=G_{0} \int \frac{T(\theta)}{\Delta \theta} d \theta \\
& =G_{0} \frac{k_{F}}{\Delta k_{y}} \int T(\theta) \cos \theta d \theta \\
& =G_{0} M\left(E_{F}\right) \frac{1}{2} \int T(\theta) \cos \theta d \theta
\end{aligned}
$$

Here we have used, mode spacing $\Delta k_{y}=2 \pi / W$, angular spacing, $\Delta \theta=\Delta k_{y} /\left(k_{F} \cos \theta\right)$ and number of modes, $M\left(E_{F}\right)=W k_{F} / \pi$. Comparing Eq. B with Eq. 3.1, we can write,

$$
T_{a v}\left(E_{F}\right)=\frac{1}{2} \int T(\theta) \cos \theta d \theta
$$

To recap, transmission through a single $p n$ junction, where the potential changes smoothly from $p$ to $n$ over a distance $2 d$ is given by,

$$
T(\theta)=e^{-\pi k_{F} d \sin ^{2} \theta}
$$


ignoring the wave-function prefactor, this is valid for moderate gate split distance $2 d$. Let us consider the $T_{a v}$ for a single split junction and a tilted junction separately.

$$
\begin{aligned}
G & \approx G_{0} M\left(E_{F}\right) \frac{1}{2} \int_{-\theta_{0}}^{\theta_{0}} d \theta e^{-\pi k_{F} d \theta^{2}} \\
& =G_{0}\left[\frac{1}{2 \sqrt{k_{F} d}}\right] M
\end{aligned}
$$

$T_{a v} \approx \frac{1}{2 \sqrt{k_{F} d}}$ with gate split. For an abrupt tilted junction,

$$
\begin{aligned}
G & \approx G_{0} \int_{-\pi / 2}^{\pi / 2-\delta} \frac{T(\theta+\delta)}{\Delta \theta} d \theta \\
& =G_{0}\left[\frac{2}{3} \cos ^{4}\left(\frac{\delta}{2}\right)\right] M
\end{aligned}
$$

due to reduced density of modes at the higher angular region, $T_{a v}=\frac{2}{3} \cos ^{4}\left(\frac{\delta}{2}\right)$ is scaled with $\delta$. Therefore, a resistance measurement $\left(R_{\text {Total }}=1 / G\right)$ will show an increase for a tilted device.

Transmission through dual tilt GPNJ device In Fig. 3.1, we have two such junctions, each of them are tilted. Individual transmissions through the junctions becomes,

$$
\begin{aligned}
& T_{1}(\theta)=e^{-\pi k_{F} d \sin ^{2}\left(\theta+\delta_{1}\right)} \\
& T_{2}(\theta)=e^{-\pi k_{F} d \sin ^{2}\left(\theta-\delta_{2}\right)}
\end{aligned}
$$

Since the tilt angle $\delta$ only modifies the angles of the incoming modes.

To get the total transmission, we combine the above two equations ignoring phase coherence [48],

$$
\begin{aligned}
\frac{1-T_{\text {eff }}}{T_{\text {eff }}} & =\frac{1}{T_{1}}+\frac{1}{T_{2}}-2 \\
& =e^{\pi k_{F} d \sin ^{2}\left(\theta+\delta_{1}\right)}+e^{\pi k_{F} d \sin ^{2}\left(\theta-\delta_{2}\right)}-2
\end{aligned}
$$


Overall transmission becomes,

$$
T_{e f f}(\theta)=\frac{1}{e^{\pi k_{F} d \sin ^{2}\left(\theta+\delta_{1}\right)}+e^{\pi k_{F} d \sin ^{2}\left(\theta-\delta_{2}\right)}-1}
$$

And

$$
\begin{aligned}
T_{a v}\left(E_{F}\right) & =\frac{1}{2} \int_{-\pi / 2}^{\pi / 2} \frac{d \theta \cos \theta}{e^{\pi k_{F} d \sin ^{2}\left(\theta+\delta_{1}\right)}+e^{\pi k_{F} d \sin ^{2}\left(\theta-\delta_{2}\right)}-1} \\
T_{a v}\left(E_{F}\right) & \approx \frac{1}{8} \frac{1}{\sqrt{k_{F} d}\left(e^{\pi k_{F} d \sin ^{2} \delta}\right)}
\end{aligned}
$$

For $\delta_{1}=\delta_{2}$.

Extracting $T_{a v}$ from transport measurement: In the experiment [2], the junction resistance was extracted from

$$
\begin{array}{r}
R j_{\text {expt }}=\left[R\left(V_{G 1}, V_{G 2}\right)+R\left(V_{G 2}, V_{G 1}\right)\right. \\
-R\left(V_{G 1}, V_{G 1}\right)-R\left(V_{G 2}, V_{G 2}\right] / 2,
\end{array}
$$

The above equation eliminates contact and device resistance due to scatterings and leaves out the resistance contribution from the $p n$ junction only. Theoretically the total resistance $R_{\text {Total }}=1 / G$ can be divided into two parts (contact and device resistance). From Eq. 3.1,

$$
\begin{aligned}
R_{\text {Total }} & =\left[G_{0}\right]^{-1} \frac{1}{M T_{a v}} \\
& =\left[G_{0}\right]^{-1}\left[\frac{1}{M}+\frac{1-T_{a v}}{M T_{a v}}\right]
\end{aligned}
$$

In presence of a $p n$ junction with non-unity $T_{a v}$, the second term can be considered as the junction resistance,

$$
R j=\left[G_{0}\right]^{-1}\left[\frac{1-T_{a v}}{M T_{a v}}\right]
$$


The theoretical $T_{a v}$ is already known (Eq. B.2). The experimental $T_{a v}$ can be found by plugging the value of $R j_{\text {expt }}$ from measurement in Eq. B.13. The only unknown value remains is the number of modes, $M=\frac{W}{\pi} \frac{\Delta E\left(V_{G}\right)}{\hbar v_{F}}$, where $\Delta E=\hbar v_{F} \sqrt{\pi C_{G} V_{G} / q}$ is the shift of Dirac point with gate voltage $V_{G}$. The gate capacitance is calculated from a simple parallel plate capacitor model $C_{G}=\frac{\epsilon}{t_{o x}}$ where gate oxide thickness $t_{o x}$ is $100 \mathrm{~nm}$. 


\section{Appendix $\mathrm{C}$}

\section{Spin filtering in TI $p n$ junction}

Let us consider a surface (top or bottom) of a TI, where an electron injected from the source at angle $\theta_{i}$ and energy $E$ is transmitted from $n$ to $p$ and collected at drain.

\section{C.1 Charge current density in TI}

Following the same procedure as graphene (Eq. A.18), we get

$$
\begin{array}{r}
J_{x}=-q v_{F} \psi^{*} \sigma_{y} \psi \\
J_{y}=q v_{F} \psi^{*} \sigma_{x} \psi
\end{array}
$$

From the TI Hamiltonian, $H=v_{F} \vec{\sigma} \times \vec{p}$, wavefunction have the form

$$
\psi=\left(\begin{array}{c}
1 \\
-i e^{i \theta}
\end{array}\right)
$$


To keep things simple, we ignore the normalizing factors. We are interested in $x$ directed charge current density. We use $\psi_{t}=t\left(\begin{array}{c}1 \\ -i e^{i \theta_{t}}\end{array}\right)$ and the transmitted current becomes

$$
J_{q t}=q v_{F}|t|^{2} \cos \theta_{t}
$$

where $\theta_{t}$ is the transmission angle for a given incident angle $\theta_{i}$. The transmission co-efficient $t$ can be calculated in the same way as graphene, taking into account both the wavefunction mismatch and tunneling contribution from evanescent modes. For a symmetric $p n$ junction, $\cos \theta_{i}=\cos \theta_{t}$, so $T=|t|^{2}$ and for smooth variation of potential, $\cos \theta_{t} T(\theta) \approx T(\theta)$ where $T(\theta)=e^{-\pi k_{F} d \sin ^{2} \theta}$. Thus Eq. C.4 can be simplified

$$
J_{q t}(\theta) \approx q v_{F}(1-R(\theta))
$$

\section{C.2 Spin current density in TI}

Let us derive the spin current operators for TI.

$$
i \hbar \frac{\partial \psi}{\partial t}=H \psi
$$

Surface states of 3D topological insulators have the following Hamiltonian (in the basis of $\mid \uparrow_{z}>$ and $\left.\mid \downarrow_{z}>\right)$

$$
H=\hbar v_{F}\left(\sigma^{x} k_{y}-\sigma^{y} k_{x}\right)
$$

$\sigma$ s are Pauli matrices. From Eq. C.6, we can write,

$$
i \hbar \frac{\partial}{\partial t}\left(\begin{array}{c}
\psi_{1} \\
\psi_{2}
\end{array}\right)=\hbar v_{F}\left(\begin{array}{cc}
0 & i k_{x}+k_{y} \\
-i k_{x}+k_{y} & 0
\end{array}\right)\left(\begin{array}{l}
\psi_{1} \\
\psi_{2}
\end{array}\right)
$$


Now using $k_{x}=-i \frac{\partial}{\partial x}$ and $k_{y}=-i \frac{\partial}{\partial y}$ we get

$$
\begin{array}{r}
i \hbar \frac{\partial \psi_{1}}{\partial t}=\hbar v_{F}\left(\frac{\partial}{\partial x}-i \frac{\partial}{\partial y}\right) \psi_{2} \\
i \hbar \frac{\partial \psi_{2}}{\partial t}=-\hbar v_{F}\left(\frac{\partial}{\partial x}+i \frac{\partial}{\partial y}\right) \psi_{1}
\end{array}
$$

\section{C.2.1 Spin current carried by $\left|\uparrow_{x}\right\rangle$}

Spin density,

$$
\begin{aligned}
& S_{x}=\psi^{*} \sigma^{x} \psi=\left(\psi_{1}^{*} \psi_{2}^{*}\right)\left(\begin{array}{cc}
0 & 1 \\
1 & 0
\end{array}\right)\left(\begin{array}{l}
\psi_{1} \\
\psi_{2}
\end{array}\right) \\
& S_{x}=\psi_{1}^{*} \psi_{2}+\psi_{2}^{*} \psi_{1}
\end{aligned}
$$

Now we take the time derivative to calculate spin current density,

$$
\frac{\partial S_{x}}{\partial t}=\psi_{1}^{*} \frac{\partial \psi_{2}}{\partial t}+\frac{\partial \psi_{1}^{*}}{\partial t} \psi_{2}+\psi_{2}^{*} \frac{\partial \psi_{1}}{\partial t}+\frac{\partial \psi_{2}^{*}}{\partial t} \psi_{1}
$$

Using the relations in Eq. C.9,

$$
\frac{\partial S_{x}}{\partial t}=-i v_{F}\left[-\psi^{*} \sigma^{z} \nabla_{x} \psi+\nabla_{x} \psi^{*} \sigma^{z} \psi\right]-v_{F}\left[\frac{\partial}{\partial y}\left(\psi_{1}^{*} \psi_{1}+\psi_{2}^{*} \psi_{2}\right)\right]
$$

Continuity equation for spin,

$$
\frac{\partial S_{x}}{\partial t}=-\left(\frac{\partial J_{x}}{\partial x}+\frac{\partial J_{y}}{\partial y}\right)+\vec{J}_{\omega}=-\vec{\nabla} \cdot \vec{J}_{s}+\vec{J}_{\omega}
$$


$\vec{J}_{\omega}$ represents the non-conservative part of spin density, usually arising from external magnetic field and spin orbit coupling. We can write,

$$
\begin{aligned}
& J_{s x}^{x}=0 \\
& J_{s y}^{x}=v_{F}\left[\left(\psi_{1}^{*} \psi_{1}+\psi_{2}^{*} \psi_{2}\right)\right]=v_{F}\left[\psi^{*} \psi\right] \\
& J_{\omega}^{x}=2 v_{F} \operatorname{Im}\left[\psi^{*} \sigma^{z} \nabla_{x} \psi\right]=0
\end{aligned}
$$

\section{C.2.2 Spin current carried by $\left|\uparrow_{y}\right\rangle$}

Similar procedure for $\left|\uparrow_{y}\right\rangle$ current gives us,

$$
\frac{\partial S_{y}}{\partial t}=-\left(\frac{\partial J_{x}}{\partial x}+\frac{\partial J_{y}}{\partial y}\right)+\overrightarrow{J_{\omega}}=-\vec{\nabla} \cdot \vec{J}_{s}+\overrightarrow{J_{\omega}}
$$

where current density $\vec{J}$ can be defined as,

$$
\begin{aligned}
J_{s x}^{y} & =v_{F}\left(\psi_{1}^{*} \psi_{1}+\psi_{2}^{*} \psi_{2}\right)=v_{F} \psi^{*} \psi \\
J_{s y}^{y} & =0 \\
J_{\omega}^{y} & =2 v_{F} \operatorname{Im}\left[\psi^{*} \sigma^{z}\left(\nabla_{y}\right) \psi\right]=0
\end{aligned}
$$

\section{C.2.3 Spin current carried by $\mid \uparrow_{z}>$}

Similar procedure for $\left|\uparrow_{z}\right\rangle$ current gives us,

$$
\frac{\partial S_{z}}{\partial t}=-\left(\frac{\partial J_{x}}{\partial x}+\frac{\partial J_{y}}{\partial y}\right)+\overrightarrow{J_{\omega}}=-\vec{\nabla} \cdot \vec{J}_{s}+\vec{J}_{\omega}
$$


So we can write,

$$
\begin{aligned}
J_{s x}^{z} & =0 \\
J_{s y}^{z} & =0 \\
J_{\omega}^{z} & =-2 v_{F} \operatorname{Im}\left[\psi^{*} \sigma^{x}\left(\nabla_{x}\right) \psi\right]+2 v_{F} \operatorname{Im}\left[\psi^{*} \sigma^{y}\left(\nabla_{y}\right) \psi\right]
\end{aligned}
$$

Again, we are only interested in $x$ component of spin currents and only $J_{s x}^{y}$ is non-zero, carried by $\mid \uparrow_{y}>$. Using Eq. C.16, the transmitted side (drain , D) and incident side (source, $S$ ) spin current densities can be written as,

$$
\begin{aligned}
& J_{s D}=-\frac{\hbar}{2} v_{F} \psi_{t}^{*} \psi_{t}=-\frac{\hbar}{2} v_{F}|t|^{2}=-\frac{\hbar}{2} v_{F}(1-R(\theta)) \\
& J_{s S}=J_{s i}+J_{s r}=-\frac{\hbar}{2} v_{F}\left(\psi_{i}^{*} \psi_{i}+\psi_{r}^{*} \psi_{r}\right)=-\frac{\hbar}{2} v_{F}\left(1+|r|^{2}\right)=-\frac{\hbar}{2} v_{F}(1+R(\theta))
\end{aligned}
$$

\section{C.3 Spin to charge current ratio}

We use $T(\theta)+R(\theta)=1$ and integrate over all angles to get the spin Hall angle $\left(\theta_{H}\right)$ at low drain bias,

$$
\begin{array}{r}
\frac{J_{s}(\theta)}{J_{q}(\theta)}=\frac{2-T(\theta)}{T(\theta)} \\
\theta_{H}=\frac{2 \int d \theta-\int T(\theta) d \theta}{\int T(\theta) d \theta}
\end{array}
$$

When the Fermi energy is at the middle of the barrier, $\hbar v_{F} k_{F}=V_{0} / 2$, we can use A.47 to simplify the integration and find

$$
\theta_{H} \approx \pi \sqrt{\frac{2 d V_{0}}{\hbar v_{F}}}-1
$$

From the expression of charge current and spin current at Drain side, the spin Hall angle for the drain side is unity. 
Total current at large drain bias is calculated from,

$$
I=\int d E D(E)\left[f_{S}(E)-f_{D}(E)\right] \int d \theta J(E, \theta)
$$

where $D$ is the density of states, $f_{S, D}$ are the Fermi-Dirac distribution of source, drain. 


\section{Bibliography}

[1] Barbaros Özyilmaz, Pablo Jarillo-Herrero, Dmitri Efetov, Dmitry A. Abanin, Leonid S. Levitov, and Philip Kim. Electronic transport and quantum hall effect in bipolar graphene $p-n-p$ junctions. Phys. Rev. Lett., 99:166804, 2007.

[2] S. Sutar, E. S. Comfort, J. Liu, T. Taniguchi, K. Watanabe, and J. U. Lee. Angledependent carrier transmission in graphene pn junctions. Nano Letters, 12(9):44604464, 2012.

[3] A. H. Castro Neto, F. Guinea, N. M. R. Peres, K. S. Novoselov, and A. K. Geim. The electronic properties of graphene. Rev. Mod. Phys., 81(1):109-162, Jan 2009.

[4] K. S. Novoselov, A. K. Geim, S. V. Morozov, D. Jiang, Y. Zhang, S. V. Dubonos, I. V. Grigorieva, and A. A. Firsov. Electric field effect in atomically thin carbon films. Science, 306(5696):666-669, 2004.

[5] S. Y. Zhou, G.-H. Gweon, J. Graf, A. V. Fedrov, C. D. Spataru, R. D. Diehl, Y. Kopelevich, D. H. Lee, Steven G. Louie, and A. Lanzara. First direct observation of dirac fermions in graphite. Nature, 2(9):595-599, 2006.

[6] S. V. Morozov, K. S. Novoselov, M. I. Katsnelson, F. Schedin, D. C. Elias, J. A. Jaszczak, and A. K. Geim. Giant intrinsic carrier mobilities in graphene and its bilayer. Phys. Rev. Lett., 100(1):016602, Jan 2008.

[7] K.I. Bolotin, K.J. Sikes, Z. Jiang, M. Klima, G. Fudenberg, J. Hone, P. Kim, and H.L. Stormer. Ultrahigh electron mobility in suspended graphene. Solid State Communications, 146(9-10):351 - 355, 2008.

[8] L Wang, I Meric, PY Huang, Q Gao, Y Gao, H Tran, T Taniguchi, K Watanabe, LM Campos, DA Muller, et al. One-dimensional electrical contact to a two-dimensional material. Science, 342(6158):614-617, 2013.

[9] Alexander A. Balandin, Suchismita Ghosh, Wenzhong Bao, Irene Calizo, Desalegne Teweldebrhan, Feng Miao, and Chun Ning Lau. Superior thermal conductivity of single-layer graphene. Nano Letters, 8(3):902-907, 2008.

[10] Changgu Lee, Xiaoding Wei, Jeffrey W. Kysar, and James Hone. Measurement of the elastic properties and intrinsic strength of monolayer graphene. Science, 321(5887):385$388,2008$. 
[11] RR Nair, P Blake, AN Grigorenko, KS Novoselov, TJ Booth, T Stauber, NMR Peres, and AK Geim. Fine structure constant defines visual transparency of graphene. Science, 320(5881):1308-1308, 2008.

[12] Supriyo Datta. Quantum transport: atom to transistor. Cambridge University Press, 2005.

[13] Melinda Y. Han, Barbaros Özyilmaz, Yuanbo Zhang, and Philip Kim. Energy band-gap engineering of graphene nanoribbons. Phys. Rev. Lett., 98:206805, May 2007.

[14] Xinran Wang, Yijian Ouyang, Xiaolin Li, Hailiang Wang, Jing Guo, and Hongjie Dai. Room-temperature all-semiconducting sub-10-nm graphene nanoribbon field-effect transistors. Phys. Rev. Lett., 100(20):206803, May 2008.

[15] Young-Woo Son, Marvin L. Cohen, and Steven G. Louie. Energy gaps in graphene nanoribbons. Phys. Rev. Lett., 97(21):216803, Nov 2006.

[16] Yang Lu and Jing Guo. Band gap of strained graphene nanoribbons. Nano Research, 3(3):189-199, 2010.

[17] Yuanbo Zhang, Tsung-Ta Tang, Caglar Girit, Zhao Hao, Michael C. Martin, Alex Zettl, Michael F. Crommie, Y. Ron Shen, and Feng Wang. Direct observation of a widely tunable bandgap in bilayer graphene. Nature, 459(7248):820-823, 2009.

[18] Xiaolin Li, Xinran Wang, Li Zhang, Sangwon Lee, and Hongjie Dai. Chemically derived, ultrasmooth graphene nanoribbon semiconductors. Science, 319(5867):1229$1232,2008$.

[19] Liying Jiao, Xinran Wang, Georgi Diankov, Hailiang Wang, and Hongjie Dai. Facile synthesis of high-quality graphene nanoribbons. Nature nanotechnology, 5(5):321-325, 2010.

[20] Frank Schwierz. Graphene transistors. Nat Nano, 5:487-496, 2010.

[21] Rui Cheng, Jingwei Bai, Lei Liao, Hailong Zhou, Yu Chen, Lixin Liu, Yung-Chen Lin, Shan Jiang, Yu Huang, and Xiangfeng Duan. High-frequency self-aligned graphene transistors with transferred gate stacks. Proceedings of the National Academy of Sciences, 109(29):11588-11592, 2012.

[22] Lei Liao, Yung-Chen Lin, Mingqiang Bao, Rui Cheng, Jingwei Bai, Yuan Liu, Yongquan Qu, Kang L. Wang, Yu Huang, and Xiangfeng Duan. High-speed graphene transistors with a self-aligned nanowire gate. Nature, 467(7313):305-308, 2010.

[23] Yanqing Wu, Vasili Perebeinos, Yu-ming Lin, Tony Low, Fengnian Xia, and Phaedon Avouris. Quantum behavior of graphene transistors near the scaling limit. Nano letters, 12(3):1417-1423, 2012.

[24] Shu-Jen Han, Dharmendar Reddy, Gary D Carpenter, Aaron D Franklin, and Keith A Jenkins. Current saturation in submicrometer graphene transistors with thin gate dielectric: Experiment, simulation, and theory. ACS nano, 6(6):5220-5226, 2012. 
[25] Jingwei Bai, Lei Liao, Hailong Zhou, Rui Cheng, Lixin Liu, Yu Huang, and Xiangfeng Duan. Top-gated chemical vapor deposition grown graphene transistors with current saturation. Nano letters, 11(6):2555-2559, 2011.

[26] Yanqing Wu, Yu-ming Lin, Ageeth A Bol, Keith A Jenkins, Fengnian Xia, Damon B Farmer, Yu Zhu, and Phaedon Avouris. High-frequency, scaled graphene transistors on diamond-like carbon. Nature, 472(7341):74-78, 2011.

[27] Anya L Grushina, Dong-Keun Ki, and Alberto F Morpurgo. A ballistic pn junction in suspended graphene with split bottom gates. Applied Physics Letters, 102:223102, 2013.

[28] Peter Rickhaus, Romain Maurand, Ming-Hao Liu, Markus Weiss, Klaus Richter, and Christian Schönenberger. Ballistic interferences in suspended graphene. Nature communications, 4, 2013.

[29] VE Calado, Shou-En Zhu, S Goswami, Q Xu, K Watanabe, T Taniguchi, GCAM Janssen, and LMK Vandersypen. Ballistic transport in graphene grown by chemical vapor deposition. Applied Physics Letters, 104(2):023103, 2014.

[30] CR Dean, AF Young, I Meric, C Lee, L Wang, S Sorgenfrei, K Watanabe, T Taniguchi, P Kim, KL Shepard, et al. Boron nitride substrates for high-quality graphene electronics. Nature nanotechnology, 5(10):722-726, 2010.

[31] F Amet, JR Williams, K Watanabe, T Taniguchi, and D Goldhaber-Gordon. Insulating behavior at the neutrality point in single-layer graphene. Physical Review Letters, 110(21):216601, 2013.

[32] Qingkai Yu, Jie Lian, Sujitra Siriponglert, Hao Li, Yong P Chen, and Shin-Shem Pei. Graphene segregated on ni surfaces and transferred to insulators. Applied Physics Letters, 93(11):113103, 2008.

[33] Jian-Hao Chen, Chaun Jang, Shudong Xiao, Masa Ishigami, and Michael S Fuhrer. Intrinsic and extrinsic performance limits of graphene devices on sio2. Nature nanotechnology, 3(4):206-209, 2008.

[34] Vadim V. Cheianov, Vladimir Fal'ko, and B. L. Altshuler. The Focusing of Electron Flow and a Veselago Lens in Graphene p-n Junctions. Science, 315(5816):1252-1255, 2007.

[35] József Cserti, András Pályi, and Csaba Péterfalvi. Caustics due to a negative refractive index in circular graphene $p$-n junctions. Phys. Rev. Lett., 99:246801, 2007.

[36] Victor Georgievich Veselago. The electrodynamics of substances with simultaneously negative values of img align $=$ absmiddle alt $=$ eps $/$ img and $\mu$. Physics-Uspekhi, 10(4):509-514, 1968.

[37] M. I. Katsnelson, K. S. Novoselov, and A. K. Geim. Chiral tunnelling and the klein paradox in graphene. Nat Phys, 2(9):620-625, 2006. 
[38] T. Low, Seokmin Hong, J. Appenzeller, S. Datta, and M.S. Lundstrom. Conductance asymmetry of graphene p-n junction. Electron Devices, IEEE Transactions on, 56(6):1292 -1299, 2009.

[39] Andrei V. Shytov, Mark S. Rudner, and Leonid S. Levitov. Klein backscattering and fabry-pérot interference in graphene heterojunctions. Phys. Rev. Lett., 101:156804, 2008.

[40] Redwan N. Sajjad and Avik W. Ghosh. High efficiency switching using graphene based electron "optics". Appl. Phys. Lett., 99(12):123101, 2011.

[41] Andrea F. Young and Philip Kim. Quantum interference and klein tunnelling in graphene heterojunctions. Nat Phys, 5(3):222-226, 2009.

[42] N. Stander, B. Huard, and D. Goldhaber-Gordon. Evidence for klein tunneling in graphene p-n junctions. Phys. Rev. Lett., 102:026807, Jan 2009.

[43] Vadim V. Cheianov and Vladimir I. Fal'ko. Selective transmission of dirac electrons and ballistic magnetoresistance of $n-p$ junctions in graphene. Phys. Rev. B, 74:041403, Jul 2006.

[44] B. Huard et al. Transport measurements across a tunable potential barrier in graphene. Phys. Rev. Lett., 98(23):236803, Jun 2007.

[45] Roman V. Gorbachev, Alexander S. Mayorov, Alexander K. Savchenko, David W. Horsell, and Francisco Guinea. Conductance of p-n-p graphene structures with airbridge top gates. Nano Letters, 8(7):1995-1999, 2008.

[46] S. Sutar, E. Comfort, and Ji Ung Lee. Incidence angle-dependent transport across a single graphene p-n junction. International Semiconductor Device Research Symposium (ISDRS), pages $1-2$, dec. 2011.

[47] Tony Low and Joerg Appenzeller. Electronic transport properties of a tilted graphene $p-n$ junction. Phys. Rev. B, 80(15):155406, Oct 2009.

[48] S. Datta. Electronic Transport in Mesoscopic Systems. Cambridge University Press, 1997.

[49] A. Svizhenko, M. P. Anantram, T. R. Govindan, B. Biegel, and R. Venugopal. Twodimensional quantum mechanical modeling of nanotransistors. Journal of Applied Physics, 91(4):2343-2354, 2002.

[50] J. W. Kłos and I. V. Zozoulenko. Effect of short- and long-range scattering on the conductivity of graphene: Boltzmann approach vs tight-binding calculations. Phys. Rev. $B, 82: 081414,2010$.

[51] N. M. R. Peres. Colloquium : The transport properties of graphene: An introduction. Rev. Mod. Phys., 82:2673-2700, 2010. 
[52] C. H. Lewenkopf, E. R. Mucciolo, and A. H. Castro Neto. Numerical studies of conductivity and fano factor in disordered graphene. Phys. Rev. B, 77:081410, 2008.

[53] M. M. Fogler, D. S. Novikov, L. I. Glazman, and B. I. Shklovskii. Effect of disorder on a graphene $p$ - $n$ junction. Phys. Rev. B, 77:075420, 2008.

[54] Redwan N Sajjad, S Sutar, JU Lee, and Avik W Ghosh. Manifestation of chiral tunneling at a tilted graphene pn junction. Physical Review B, 86(15):155412, 2012.

[55] Redwan N Sajjad, Carlos A Polanco, and Avik W Ghosh. Atomistic deconstruction of current flow in graphene based hetero-junctions. Journal of Computational Electronics, $12: 232-247,2013$.

[56] Qing Hua Wang and Mark C. Hersam. Room-temperature molecular-resolution characterization of self-assembled organic monolayers on epitaxial graphene. Nat Chem, 1(3):206-211, 2009.

[57] Dmitri E Nikonov and Ian A Young. Uniform methodology for benchmarking beyondcmos logic devices. In Electron Devices Meeting (IEDM), 2012 IEEE International, pages 25-4. IEEE, 2012.

[58] Wei Zhao and Yu Cao. New generation of predictive technology model for sub-45 nm early design exploration. Electron Devices, IEEE Transactions on, 53(11):2816-2823, 2006.

[59] Xiao-Liang Qi and Shou-Cheng Zhang. Topological insulators and superconductors. Reviews of Modern Physics, 83(4):1057, 2011.

[60] B Andrei Bernevig, Taylor L Hughes, and Shou-Cheng Zhang. Quantum spin hall effect and topological phase transition in hgte quantum wells. Science, 314(5806):1757-1761, 2006.

[61] Y Xia, Dong Qian, David Hsieh, L Wray, A Pal, Hsin Lin, Arun Bansil, DHYS Grauer, YS Hor, RJ Cava, et al. Observation of a large-gap topological-insulator class with a single dirac cone on the surface. Nature Physics, 5(6):398-402, 2009.

[62] Supriyo Datta, Vinh Quang Diep, and Behtash Behin-Aein. What constitutes a nanoswitch? a perspective. arXiv preprint arXiv:1404.2254, 2014.

[63] Supriyo Datta, Sayeef Salahuddin, and Behtash Behin-Aein. Non-volatile spin switch for boolean and non-boolean logic. Applied Physics Letters, 101(25):-, 2012.

[64] MI Dyakonov and VI Perel. Current-induced spin orientation of electrons in semiconductors. Physics Letters A, 35(6):459-460, 1971.

[65] JE Hirsch. Spin hall effect. Physical Review Letters, 83(9):1834, 1999.

[66] Ming-Hao Liu and Klaus Richter. Efficient quantum transport simulation for bulk graphene heterojunctions. Physical Review B, 86(11):115455, 2012. 
[67] Chi-Feng Pai, Luqiao Liu, Y Li, HW Tseng, DC Ralph, and RA Buhrman. Spin transfer torque devices utilizing the giant spin hall effect of tungsten. Applied Physics Letters, 101(12):122404, 2012.

[68] O. Mosendz, J. E. Pearson, F. Y. Fradin, G. E. W. Bauer, S. D. Bader, and A. Hoffmann. Quantifying spin hall angles from spin pumping: Experiments and theory. Phys. Rev. Lett., 104:046601, Jan 2010.

[69] B. Gu, I. Sugai, T. Ziman, G. Y. Guo, N. Nagaosa, T. Seki, K. Takanashi, and S. Maekawa. Surface-assisted spin hall effect in au films with pt impurities. Phys. Rev. Lett., 105:216401, Nov 2010.

[70] WJ Liu, HY Yu, SH Xu, Q Zhang, X Zou, JL Wang, KL Pey, J Wei, HL Zhu, and MF Li. Understanding asymmetric transportation behavior in graphene field-effect transistors using scanning kelvin probe microscopy. Electron Device Letters, IEEE, 32(2):128-130, 2011.

[71] Ioan Mihai Miron, Kevin Garello, Gilles Gaudin, Pierre-Jean Zermatten, Marius V Costache, Stéphane Auffret, Sébastien Bandiera, Bernard Rodmacq, Alain Schuhl, and Pietro Gambardella. Perpendicular switching of a single ferromagnetic layer induced by in-plane current injection. Nature, 476(7359):189-193, 2011.

[72] Y. Niimi, Y. Kawanishi, D. H. Wei, C. Deranlot, H. X. Yang, M. Chshiev, T. Valet, A. Fert, and Y. Otani. Giant spin hall effect induced by skew scattering from bismuth impurities inside thin film cubi alloys. Phys. Rev. Lett., 109:156602, Oct 2012.

[73] AR Mellnik, JS Lee, A Richardella, JL Grab, PJ Mintun, MH Fischer, A Vaezi, A Manchon, E-A Kim, N Samarth, et al. Spin transfer torque generated by the topological insulator bi_2se_3. arXiv preprint arXiv:1402.1124, 2014.

[74] Vadim V Cheianov, Vladimir Fal'ko, and BL Altshuler. The focusing of electron flow and a veselago lens in graphene pn junctions. Science, 315(5816):1252-1255, 2007.

[75] T. Low, Seokmin Hong, J. Appenzeller, S. Datta, and M.S. Lundstrom. Conductance asymmetry of graphene p-n junction. Electron Devices, IEEE Transactions on, 56(6):1292-1299, 2009.

[76] J. R. Williams, Tony Low, M.S. Lundstrom, and C.M. Marcus. Gate-controlled guiding of electrons in graphene. Nat Nano, 6(4):222-225, 2011.

[77] K. M. Masum Habib, Redwan N. Sajjad, and Avik Ghosh. Chiral tunneling of topological states for giant longitudinal spin hall angle. arXiv preprint arXiv:1402.6222, 2014.

[78] Supriyo Datta. A new perspective on transport. 2012.

[79] Rolf Landauer. Spatial variation of currents and fields due to localized scatterers in metallic conduction. IBM Journal of Research and Development, 1(3):223-231, 1957. 
[80] F. Tseng, D. Unluer, K. Holcomb, M. R. Stan, and A. W. Ghosh. Diluted chirality dependence in edge rough graphene nanoribbon field-effect transistors. Applied Physics Letters, 94(22):223112, 2009.

[81] Michael Galperin, Sivan Toledo, and Abraham Nitzan. Numerical computation of tunneling fluxes. The Journal of Chemical Physics, 117(23):10817-10826, 2002.

[82] Khairul Alam and Roger K Lake. Leakage and performance of zero-schottky-barrier carbon nanotube transistors. Journal of applied physics, 98(6):064307-064307, 2005.

[83] Fengnian Xia, Vasili Perebeinos, Yu-ming Lin, Yanqing Wu, and Phaedon Avouris. The origins and limits of metal-graphene junction resistance. Nature nanotechnology, 6(3):179-184, 2011.

[84] Redwan N. Sajjad and Avik W. Ghosh. Manipulating chiral transmission by gate geometry: Switching in graphene with transmission gaps. ACS Nano, 7(11):9808-9813, 2013.

[85] PA Khomyakov, AA Starikov, G Brocks, and PJ Kelly. Nonlinear screening of charges induced in graphene by metal contacts. Physical Review B, 82(11):115437, 2010.

[86] Joshua A Robinson et. al. Contacting graphene. Applied Physics Letters, 98(5):053103053103, 2011.

[87] P Blake et. al. Influence of metal contacts and charge inhomogeneity on transport properties of graphene near the neutrality point. Solid State Communications, 149(27):10681071, 2009.

[88] Yang Sui, Tony Low, Mark Lundstrom, and Joerg Appenzeller. Signatures of disorder in the minimum conductivity of graphene. Nano letters, 11(3):1319-1322, 2011.

[89] J.-H. Chen, C. Jang, S. Adam, M. S. Fuhrer, E. D. Williams, and M. Ishigami. Charged impurity scattering in graphene. Nat Phys, 4:377-381, 2008.

[90] K. S. Novoselov, A. K. Geim, S. V. Morozov, D. Jiang, M. I. Katsnelson, I. V. Grigorieva, S. V. Dubonos, and A. A. Firsov. Two-dimensional gas of massless dirac fermions in graphene. Nature, 438(7065):197-200, 2005.

[91] Yuanbo Zhang, Yan-Wen Tan, Horst L. Stormer, and Philip Kim. Experimental observation of the quantum hall effect and berry's phase in graphene. Nature, 438(7065):201$204,2005$.

[92] Edward McCann and Vladimir I. Fal'ko. Landau-level degeneracy and quantum hall effect in a graphite bilayer. Phys. Rev. Lett., 96(8):086805, Mar 2006. 